\title{
SPUTTERING OF THE GALIIUM-INDIUM \\ EUTECTIC ALLOY IN THE LIQUID PHASE
}

\author{
Thesis by \\ Mark Frederick Dumke
}

In Partial Fulfillment of the Requirements

for the Degree of

Doctor of Philosophy
California Institute of Technology Pasadena, California

1982

(Submitted October 7, 1981) 
ACKNOWLEDGMENTS

I would like to thank Tom Tombrello and Bob Weller who were the pillar of cloud by day and the pillar of fire by night for this work. Their faithful guidance and inspiration are the true causes for the end of years of wandering through the wilderness of graduate school.

Special thanks are due to Bob Housley, Joe Griffith, and Barbara Cooper who shared their considerable expertise with me on numerous occasions. Yuanxun Qiu shared not only his expertise, but also his time on more sleepless nights than either of us care to number. His advice, inexharstible help, and kindness will not be soon forgotten.

Finally, I would like to thank wil Schick who should have been decorated many times for going above and beyond the call of duty. 


\section{ABSTRACT}

We have measured sputtering yields and angular distributions of sputtered atoms from both the solid and liquid phases of gallium, indium, and the gallium-indium eutectic alloy。 This was done by Rutherford backscattering analysis of graphite collector foils. The solid eutectic target shows a predominance of indium crystallites on its surface which have to be sputtered away before the composition of the sputtered atoms equals the bulk target composition. The size of the crystallites depends upon the conditions under which the alloy is frozen. The sputtering of the liquid eutectic alloy by $15 \mathrm{keV} \mathrm{Ar}^{+}$results in a ratio of indium to gallium sputtering yields which is 28 times greater than would be expected from the target stoichiometry. Furthermore, the angular distribution of gallium is much more sharply peaked about the normal to the target surface than the indium distribution. When the incident $\mathrm{Ar}^{+}$energy is increased to $25 \mathrm{keV}$, the gallium distribution broadens to the same shape as the indium distribution. With the exception of the sharp gallium distribution taken from the liquid eutectic at $15 \mathrm{kev}$, all angular distributions from liquid targets fit a $\cos ^{2} \theta$ function. An ion-scattering-spectroscopy analysis of the liquid eutectic alloy reveals a surface layer of almost pure indium. A thermodynamic explanation for this highly segregated layer is discussed. The liquid eutectic alloy provides us with a unique target system which allows us to estimate the fraction of sputtered material which comes from the first monolayer of the surface. 
TABLE OF CONTENTS

I. Introduction 1

II. Apparatus 5

A. Sputtering Apparatus 5

B. Rutherford Backscattering Apparatus 8

III. Procedure 10

$\begin{array}{ll}\text { A. Targets } & 10\end{array}$

B. Beam Current Integration 15

C. Collector Foils 18

D. Rutherford Backscattering 21

E. Data Analysis 25

IV. Results 29

A. Elemental Gallium and Indium 29

B. Iiquid Gallium-Indium Eutectic Alloy 32

C. Solid Gallium-Indium Eutectic Alloy 37

D. Ion Scattering Spectroscopy 41

v. Discussion 44

Appendix - Ion Scattering Spectroscopy Apparatus 52

References $\quad 55$

$\begin{array}{ll}\text { Tables } & 57\end{array}$

$\begin{array}{ll}\text { Figures } & 65\end{array}$ 


\section{INTRODUCTION}

To date, sputtering experiments have been almost completely restricted to solid targets. Only three publications concerned with the sputtering of liquids are extant (Krutenat and Panzera, 1970; Krutenat and Geswick, 1970; Garvin, 1968), and only one of these experiments was done with a target-chamber pressure lower than $10^{-6}$ torr (Garvin, 1968). Consequently, only one of these publications seems free of the serious effect which surface contamination can have on the measurement of sputtering yields. This one work, conducted by H. L. Garvin, deals solely with pure elemental targets and concerns itself exclusively with relative, not absolute, sputtering yields. In addition to these three publications, a talk was given in July of 1981 at the Ninth International Conference of Atomic Collisions in Solids which described photon emission from atoms sputtered from liquid elemental targets (Gabta, 1981).

There are two motivations for entering into the rather pristine frontier of liquid sputtering. First of all, one would like to know how the angular distribution of sputtered atoms is affected by a phase change in the target. Secondly, liquid sputtering can shed light on the phenomenon of surface segregation in non-elemental targets.

Collision-cascade theory predicts that the angular distribution of the sputtered atoms should have a $\cos \theta$ dependence (Signund, 1980), where $\theta$ is the angle with respect to the target normal. However, several factors can affect 
the angular distribution such as surface roughness, target anisotropy, and the texture of polycrystalline targets. These effects should not play a role in liquid sputtering.

The phenomenon of sputter-induced surface segregation has been observed for a variety of binary solid targets (Liau et al., 1978). The composition of the target surface changes during sputtering. Characteristically, the surface composition arrives at a steady state after the removal of a few hundred monolayers of target material. After this steady state is reached, sputtered atoms are removed from the target in a ratio equal to the bulk composition ratio.

Some theoretical work has been done on surface segregation. One theory which has been successful in predicting the nature of several surface segregations states that the heavier atoms should always be enriched at the surface (Haff, 1977). This theory assumes an equipartition of energy between the atoms at the surface and concludes that lighter atoms are sputtered more easily than heavier atoms. However, since equipartition of energy implies thermal equilibrium, one would expect this theory to apply only to thermal-spike sputtering. A second theory of surface segregation contends that whichever kind of atom is most abundant in the bulk composition will be sputtered preferentially. This theory is based on the fact that collisions between atoms of like mass transfer energy more efficiently than collisions between atoms of different masses. While this second theory makes a more careful analysis of the collision cascade, it does not successfully predict the 
surface segregations for several binary targets (Liau et al., 1978). The reason for this is that the second theory does not account for differences in binding potential between the two target components. The theory is successful in describing the fractionation of calcium isotopes where the binding potential is the same for both ${ }^{4 a} \mathrm{Ca}$ and ${ }^{44} \mathrm{Ca}$ (Watson and Haff, 1979) •

While the sputtering of binary solid targets is required to reach a steady state in which the composition of the sputtered atoms is the same as the bulk target composition, no such requirement pertains to liquid targets. In solid targets, when one kind of atom is sputtered preferentially, the other kind of atom is left enriched at the target surface. But when one kind of atom is sputtered preferentially in a liquid target, atoms of the same kind can continue to diffuse from the bulk to the surface where they themselves can also be sputtered preferentially. Consequently, atoms sputtered from liquid binary targets can have a composition which is very different from the bulk composition. Ideally, this difference should be independent of sputtering dose until the bulk of the target is actually depleted of the component which is preferentially sputtered. Under the experimental conditions which will be described here, a typical target would have to be sputtered continuously for years before such a bulk depletion could occur.

One further theoretical consideration which should be made is that sputtering yields are independent of target density. 
Collision-cascade theory results in the following expression for sputtering yields:

$$
S(E)=\frac{0.042}{U} \frac{1}{\rho} \frac{d E}{d x}
$$

$U$ is the surface binding energy, $\rho$ is the target density, and $\frac{d E}{d x}$ is the nuclear stopping power which is proportional to the target density (Sigmund, 1980). Ideally, the density independence of sputtering yields should apply equally well to both solid and liquid targets. It should be noted that theoretical work done on the sputtering of gases predicts that the sputtering yield should be independent of target density even for gaseous targets (Haff and Watson, 1979). (Note that no experimental data exist for gaseous targets.) Therefore, differences in sputtering behavior due to phase change are difficult to attribute merely to target density differences. 


\section{APPARATUS}

\section{A. Sputtering Apparatus}

One of the most compelling reasons why more work has not been done on the sputtering of liquids is the difficulty in holding a liquid target. This difficulty arises from the fact that most ion accelerators produce a horizontal beam. The problem can be surmounted by constructing an accelerator which produces a vertical analyzed beam.

Figures 1 and 2 show the accelerator which was set up in order to sputter liquid targets. Ions are produced in the radio-frequency ion source which is held at a potential several kilovolts above ground (Figure 3). Naturally, all the power supplies which produce the radio-frequency discharge, the anode voltage, and the source-magnet current also have to be maintained at this potential and are powered through an isolation transformer. The Pierce electrode which is used to extract the beam from the source is at ground potential. An einzel lens just downstream of the Pierce electrode is used for focussing. The accelerator vacuum chamber is pumped by a cryobaffled diffusion pump and is isolated from the target chamber by an in-line cryotrap. This arrangement permits the pressure inside the source bottle to be $50 \mu$ while never allowing the pressure inside the target chamber to rise above $3 \times 10^{-8}$ torr. The analyzing magnet is double-focussing, and the strength of the magnetic field is measured by means of a Hall probe. The distance from the exit end of the magnet 
to the final focus is approximately described by:

$$
d=R \sqrt{2} \cot \frac{\theta}{\sqrt{2}}
$$

$R$ is the radius of curvature of the magnet and $\theta$ is the angle through which the magnetic field causes the beam to turn. Using this relation which assumes a parallel beam at the entrance of the magnet, the distance to final focus is approximately $28.5 \mathrm{~cm}$. The position of the target is arranged to correspond to this distance. The target chamber is pumped by a cryopump which typically achieves base pressures of $7 \times 10^{-9}$ torr.

At the top of the target chamber is a rotary feedthrough which holds the collector-foil apparatus. There are four sets of collector foils which can be positioned over the target by means of the rotary feedthrough. The rotary feedthrough is equipped with a spring-loaded retention system which facilitates the positioning of the feedthrough at four locations $90^{\circ}$ apart. This apparatus allows several sputtering experiments to be conducted without opening the target chamber. The collector-foil holders are semicircular and are designed to have their centers on the target surface. Each set of collector-foil holders has a $6 \mathrm{~mm}$. hole in it which allows the incident beam to reach the target.

Figure 4 illustrates the target-holder apparatus which resembles a miniature "bird bath." A tantalum dish is used to hold the target material since unlike most metals, tantalum is resistant to amalgamation with gallium and indium. The 
tantalum dish shown in the figure is held in place by means of a tight friction fit. Since this makes it difficult to remove the dish without distorting it, another smallerdiameter dish held in place by a clamp has also been used. A cavity inside the main body of the target holder contains an Analog Devices temperature transducer. This cavity is machined to close tolerances to hold the temperature transducer directly beneath the tantalum dish and to achieve good thermal contact between the transducer and the target holder. A tungsten heating filament is wrapped about the base of the target holder. The heating filament is encased in a Macor block which completely shields the target holder from the filament and prevents the passage of the thermal electrons from the filament to the holder. If electrons from the filament were allowed to strike the holder, they would interfere with the integration of the beam current impinging on the target. The entire target holder is biased at $+300 \mathrm{~V}$ as well as the catcher-foil holder directly above it. This makes the entire system in Figure 4 a Faraday cup for beam-integration purposes. The target holder and the collector-foil holder can be electrically isolated from each other in order to measure target current and collector current separately. Figure 5 shows the temperature-controller circuit used to set the target temperature. The temperature transducer puts out a current which is proportional to its absolute temperature. Therefore, the temperature is measured by monitoring the voltage across the $10 \mathrm{k} \Omega$ wire-wound resistor 
which is connected to the negative end of the transducer. The circuit contains a Kelvin-Varley potentiometer which provides a stable, adjustable voltage and which has both coarse and fine controls. The output of the Kelvin-Varley potentiometer is compared to the buffered output of the temperature transducer in order to regulate the current passing through the heating coil. A buffer op amp is required to guard the $10 \mathrm{k} \Omega$ wire-wound resistor against any feedback current from the op amp which does the regulating. This circuit can maintain the temperature of the target assembly within $\pm 0.01^{\circ} \mathrm{C}$ for 24 hours with no noticeable drift.

\section{B. Rutherford Backscattering Apparatus}

The Rutherford backscattering arrangement is shown in Figure 6. The incident beam of $5.00 \mathrm{MeV}{ }^{19} \mathrm{~F}^{2+}$ is obtained from the CIT-ONR tandem accelerator. The collector foils are removed from the curved holder shown in Figure 1 and are placed in the twelve-sided holder shown in Figure 6. This twelve-sided holder can be rotated to position each collector foil in the way of the incident beam. It can also be moved perpendicularly to the plane of the diagram in order to allow us to inspect several different points on each collector foil. For this purpose, the holder is mounted on a linear feedthrough which can be positioned reproducibly to within $0.1 \mathrm{~mm}$. Furthermore, the foils can be reproducibly situated on the holder to within $0.02 \mathrm{~mm}$. by means of a ridge on the holder which retains the ends of the foils. The target holder 
$-9-$

is surrounded by a negatively biased screen which repels any electrons which may be traveling along with the incident beam. This screen also aids in the suppression of secondary electrons which are knocked off of the target by the incident beam. The screen has a hole in it which allows the incident beam to pass through and which also provides a clear pathway from the target to the detector. Most ultraviolet radiation emitted from the target should pass through the screen without producing tertiary electrons since the screen submits a very small surface area to bombardment by secondary radiation from the target. 
III. PROCEDURE

\section{A. Targets}

The sputtering of the solid and liquid phases of pure gallium, pure indium, and the eutectic alloy of gallium and indium was investigated. All target metals were at least 99.9999\% pure and were purchased from the Thiokol/Ventron Division of Alpha Products. Since both gallium and indium surfaces oxidize readily in air, all target material was stored in an argon-filled glove box. The glove box was maintained at positive pressure during all transfers of target material into or out of the box. Each target was stored in its own polyethylene container within the glove box, and each of these containers was flooded with argon and closed before shutting off the flow of argon in the glove box. Targets were transferred as quickly as possible from the glove box to the UHV target chamber. The UHV chamber was always vented with dry nitrogen, and the target material typically sat in this atmosphere for 20 minutes before the chamber could be closed up and roughed out. The surfaces of all liquid targets were skimmed with a disposable piece of polyethylene shortly before closing the chamber. This was done in order to remove any oxide which may have formed. When this procedure was practiced with targets which were allowed to form visible oxide films, it was found that the film invariably floated on the target surface and that it readily stuck to polyethylene. 
The argon-filled glove box mentioned above was also used to calibrate the temperature transducer. The entire target holder depicted in Figure 4 was placed inside the glove box so that the melting points of targets could be determined without oxidizing them. The heater current required to melt a target in the glove box was typically much higher than the current required to melt the same target in vacuum because of convection. However, the temperature-controller circuit shown in Figure 5 did not require a constant heater current in order to calibrate the transducer since the ultimate transducer temperature depended only upon the setting of the Kelvin-Varley potentiometer.

After the target material had been lowered into the UHV chamber and the eight-inch-diameter flange containing the collector-foil apparatus had been replaced, the chamber was immediately roughed out using a sorption pump. Typically, the sorption pump lowered the pressure in the chamber to $4 \mu$ in 45 minutes. Then the in-line cryotrap was filled with liquid nitrogen, and the straight-through valve between the cryotrap and the analyzing magnet was opened. In this way, the chamber could be roughed out further over a long period of time (eight to twelve hours). This long roughing period is recommended for cryopumped systems because of the enormous surface area in the cryopump which is covered with adsorbed gas molecules. The pressure in the chamber was usually $5 \times 10^{-4}$ torr before the cryopump was started and the straight-through valve was closed. 75 minutes after turning on the cryopump compressor, 
the temperature of the baffled cryosurfaces was $14^{\circ} \mathrm{K}$. A base pressure of $7 \times 10^{-9}$ torr was usually reached approximately one week after the cryopump was started. The base pressure in the diffusion-pumped accelerator chamber was typically $3 \times 10^{-7}$ torr. During sputtering runs, the pressure in the accelerator chamber was $1.0 \times 10^{-6}$ torr due to the admission of argon gas into the source bottle through a needle valve. In order to allow the $\mathrm{Ar}^{+}$beam into the target chamber, the straightthrough valve which separated the diffusion-pumped vacuum system from the cryopumped system had to be opened. However, the in-line cryotrap never allowed the pressure in the target chamber to rise above $3 \times 10^{-8}$ torr. Argon gas was the major component of this pressure since turning off the ion source and closing the argon needle valve allowed the pressure inside the target chamber to return to $7 \times 10^{-9}$ torr.

The beam most frequently used for sputtering was $15 \mathrm{keV}$ $\mathrm{Ar}^{+}$. The beam current on target was characteristically $0.6 \mu \mathrm{A}$ and an aperture upstream of the target-collector system shown in Figure 4 confined the beam to a $3 \mathrm{~mm}$. diameter spot. If a sputtering yield of 3 is assumed, which is lower than any total yield actually measured, then one monolayer of target material was removed every 10 seconds. At a target chamber pressure of $3 \times 10^{-8}$ torr, gas molecules impinged on all the chamber surfaces at a rate of one monolayer every 30 seconds. Thus target atoms were removed at a rate three times faster than the rate at which gas molecules impinged on the target surface. This means that once the target surface was cleaned, 
it remained clean during the course of the sputtering runs. This argument for surface cleanliness has not invoked the fact that the $3 \times 10^{-8}$ torr pressure was comprised mostly of argon which does not readily stick to surfaces. Furthermore, even the $7 \times 10^{-9}$ torr base pressure probably had a large helium component since the cryopump does not pump helium efficiently. Helium, like argon, has a low enthalpy of adsorption for most surfaces.

Normally before any sputtering experiments were conducted, the targets were sputter-cleaned with $1.00 \mathrm{mCoulomb}$ of incident $15 \mathrm{keV} \mathrm{Ar}^{+}$. This was enough dose to remove approximately 200 monolayers of target material. A brief experiment to test target cleanliness was conducted at the Rockwell International Corporation Science Center in Thousand Oaks, California. There a liquid target of the eutectic alloy of gallium and indium was allowed to oxidize in air for two hours. Then the target was placed into a UHV chamber and its surface was viewed using a scanning electron microscope. Then it was sputter-cleaned with a dose of $5 \mathrm{keV} \mathrm{Ar}{ }^{+}$sufficient to remove approximately 50 monolayers. After the sputter-cleaning, the electron microscope no longer displayed any of the light patches which are characteristic of oxides in the region where the $5 \mathrm{keV} \mathrm{Ar}+$ beam impinged. The target remained free of oxides overnight for 16 hours in a vacuum of $3 \times 10^{-10}$ torr before it had to be removed.

Gallium and indium were chosen to be the target elements not only because of their low melting points $\left(29.78^{\circ} \mathrm{C}\right.$ for 
gallium, $156.61^{\circ} \mathrm{C}$ for indium, $15.7^{\circ} \mathrm{C}$ for the eutectic alloy), but also because of their low vapor pressures. Gallium has to be heated to a temperature of $500^{\circ} \mathrm{C}$ before its vapor pressure reaches $1 \times 10^{-10}$ torr (Cochran and Foster, 1962). At room temperature, the vapor pressure of gallium is only $2 \times 10^{-37}$ torr while the vapor pressure of indium is $4 \times 10^{-33}$ torr (Nesmeyanov, 1963). These low vapor pressures not only allowed the attainment of low target-chamber pressures, but they also ruled out the possibility of bulk target evaporation since no target was ever heated above $164^{\circ} \mathrm{C}$ and since the beam only deposited $9 \mathrm{~mW}$ of power into the target. Nevertheless, for each set of sputtering runs, one position of the collectorfoil carousel was devoted to holding blank collector foils. These foils were positioned over the target whenever beam was not impinging on it. Therefore, they were used to monitor whether any contaminants were being introduced by handling the foils as well as whether any target atoms were reaching the collector foils by any process other than sputtering. These blank foils never showed any detectable levels of either gallium or indium.

In order to make targets suitable for the holder shown in Figure 4, the target material had to be poured into a tantalum dish. This procedure was carried out for gallium and the eutectic alloy of gallium and indium in the argonfilled glove box mentioned above. However, the pure indium target was made by melting indium pellets in a bell jar at a pressure of $1 \times 10^{-6}$ torr. This was done to insure that the 
hot, molten indium would not form a thick oxide layer on the surface. After resolidifying, the indium target was allowed to cool for 20 minutes before the bell jar was vented and the target removed. The indium target was immediately transferred to the UHV target chamber where it was to be sputter-cleaned later as described above.

While in the UHV chamber, targets were heated using the tungsten filament depicted in Figure 4. In order to solidify the eutectic alloy of gallium and indium, a method of cooling targets was also required. Consequently, a copper strap was wrapped tightly around the outermost baffle of the cryopump, and one end of the strap was attached to the target holder assembly. This strap served to cool the target holder down to $8^{\circ} \mathrm{C}$ which was sufficient to keep the eutectic alloy from melting after it had solidified. However, as experiments conducted outside the chamber demonstrated, the liquid eutectic remained in superfusion without a seed crystal until it was cooled below $0^{\circ} \mathrm{C}$. This supercritical behavior has been documented for gallium and gallium alloys (De la Breteque, 1962). In order to cool the target assembly further, a dry ice pack was prepared and simply placed on the flange which supported the assembly. After the dry ice pack had been maintained for 24 hours, the temperature of the target holder was $-20^{\circ} \mathrm{C}$.

B. Beam Current Integration

As stated earlier, both the target assembly and the collector-foil holder assembly were biased at $+300 \mathrm{~V}$, and the 
outputs of both were fed into the same current digitizer. In order to keep sputtered gallium and indium atoms from getting all over the inside of the target chamber, each set of collectorfoil holders was covered with a hemispherical tent made out of aluminum foil. These tents were in good electrical contact with the rest of the collector-foil holder assembly. As a result of this arrangement, secondary electrons emitted from the target were subtended with a solid angle of almost $2 \pi$ by the collector-foil assembly. Since the collector-foil assembly was also positively biased, tertiary electrons emitted from its surface were not allowed to escape the Faraday cup constituted by the target-collector system. A bias of $+600 \mathrm{v}$ was also tried and resulted in the same measured beam current within 38 , which was within the limits of reproducibility caused by fluctuations in the ion source output. This was done to insure that the $+300 \mathrm{~V}$ bias provided enough electron suppression.

One serious concern which remained was that the $15 \mathrm{keV}$ $\mathrm{Ar}^{+}$beam might have been knocking sizeable numbers of electrons off the aperture which was just upstream of the collector-foil holder. These electrons would then be attracted to the positively biased target-collector Faraday cup and would cause the beam current measurement to be too low. In order to estimate the size of this effect, the target was biased at $+600 \mathrm{~V}$, and the collector-foil was left biased at $+300 \mathrm{~V}$. The target current and the collector-foil current were measured separately. The target current remained unchanged from the 
previous beam current measurement, and the collector-foil current was only $-4 \mathrm{nA}$. Since the target was positively biased with respect to the collector-foil holder, the $-4 \mathrm{nA}$ represented electrons which were being ejected from the rim of the aperture instead of secondary electrons from the target. The total beam current at this time was $0.40 \mu \mathrm{A}$, so the electrons ejected from the aperture constituted an error in beam current integration of 18 .

Another test of beam current integration was conducted with a target bias of $+300 \mathrm{~V}$ and a collector-foil holder bias of $-300 \mathrm{~V}$. Under these conditions, the target current again remained unchanged within $3 \%$ while the collector-foil current was $+20 \mathrm{nA}$, which indicated bombardment by sputtered ions as well as the emission of tertiary electrons from the negatively biased collector-foil holder. Although the collector-foil holder was protected from secondary electrons emitted from the target since such electrons would have to overcome a $600 \mathrm{v}$ potential barrier; energetic photons, hot neutrals, and positive ions could still travel from the target to the collector-foil holder and induce tertiary electron emission.

The possibility that the $+300 \mathrm{~V}$ suppression on the targetcollector system could noticeably defocus the $15 \mathrm{keV} \mathrm{Ar}^{+}$beam was also explored by examining a solid gallium target which had been bombarded by 15 mCoulombs of incident $\mathrm{Ar}^{+}$ions. The target exhibited a shallow crater $3 \mathrm{~mm}$. in diameter which indicated that the beam was not diverging significantly after passing through the aperture which was upstream of the collector-foil holder. 
The reliability of the current digitizer was checked using a calibrated current source. This check was made while the ion source was running to insure that the digitizer was not affected by any radio-frequency interference originating from the oscillator used to obtain the discharge in the source. Furthermore, thermionic emission from the heater filament was checked after the liquid indium run when the heater current was the highest ever used. The total current going into the digitizer with no incident beam on target was $-3 \mathrm{nA}$, which constituted an error in beam current integration of 0.58 .

It should be noted that with the beam current integration scheme finally settled upon (i.e., both target and collectorfoil holder at $+300 \mathrm{~V})$, the sputtered atoms traveled in a region essentially free of electric fields. Consequently, the angular distribution of sputtered ions or sputtered atoms in excited states with high electric dipole moments should not have been disturbed by the electron suppression.

\section{Collector Foils}

The collector foils were normally made out of $0.13 \mathrm{~mm}$. thick graphite sheets which were obtained from Graphite Machine Products and which contained less than 5 parts per million of impurities. These foils could be bent to conform to the curved collector-foil holders which are depicted in Figures 1 and 4. However, some care had to be taken to bend the foils without breaking them since they were quite brittle. When the foils were removed from the curved holders, they 
immediately returned to a straight configuration which allowed them to be placed easily into the Rutherford backscattering holder shown in Figure 6.

Once sputtered atoms were ejected from the target and traversed the distance between target and collector foil, there was no guarantee that they would all stick to the collector foils. Consequently, several different types of collector foils were compared simultaneously with the graphite foils to determine if the sticking fraction of sputtered atoms was different. First of all, aluminum and graphite foils were simultaneously used to collect atoms sputtered from a liquid eutectic target. Then both aluminum and graphite foils were again used simultaneously over a pure indium target. The number of gallium and indium atoms collected by the aluminum foils corresponded well to the number of atoms collected by the graphite foils in light of the $5 \%$ experimental uncertainty. Then collector foils made up of more massive atoms than the sputtered atoms were used. This was done in order to discover if the sputtered atoms would recoil from heavier collectorfoil atoms. Tantalum collector foils were tried and then abandoned because it was impossible to detect atoms lighter than tantalum with Rutherford backscattering due to the large tantalum thick-target yield. In order to get around this problem, graphite foils with an evaporated $2.5 \mu \mathrm{g} . \mathrm{cm}^{-2}$ coating of gold were used. Sputtering of the liquid eutectic target again revealed no significant differences between the gold-coated graphite and the uncoated graphite foils in the 
ability to collect gallium and indium atoms. Finally, graphite foils with a $2.0 \mu \mathrm{g} . \mathrm{cm}^{-2}$ coating of indium were used to collect atoms sputtered from a pure gallium target. This was done to see if there was any reaction between gallium and indium which would affect the sticking probability. Once again there was no discernible difference between the coated and uncoated foils. Table 1 . summarizes the results of the collector-foil experiments. The ratios of the concentrations of sputtered atoms on various foils to their concentrations at the corresponding locations on pure graphite foils are recorded. A correction needed to be made for the aluminum collectors since they were not the same thickness as the graphite, and therefore their surfaces were not the same distance away from the target. The use of foils coated with gold and indium also demonstrated that the sputtered atoms were not noticeably diffusing into the graphite since the coatings should have acted as barriers to this diffusion and therefore should have shown higher concentrations of sputtered atoms than the uncoated foils. The possibility of diffusion was further explored by analyzing the same collector foil at two different times which were six months apart. The peaks in the two backscattering spectra not only were of the same size but also the same shape.

A further test of the ability of the collector foils to collect sputtered atoms was conducted by sputtering a liquid eutectic target with different doses of incident $\mathrm{Ar}^{+}$. A sputtering run with an $\mathrm{Ar}^{+}$dose of 5 mCoulombs was followed by a sputtering run with a new set of collector foils and an $\mathrm{Ar}^{+}$ 
dose of 10 mCoulombs. Then corresponding locations on the two sets of collector foils were analyzed via Rutherford backscattering. The collector foils from the second run contained 1.98 times as much gallium and 1.88 times as much indium as the foils from the first run which was in keeping with the doubled dose and the $5 \%$ experimental error. During the course of all the sputtering runs described here, no location on any collector foil was ever required to collect more than ten monolayers of sputtered atoms.

Since the targets used in this experiment were either liquids or frozen liquids, it was difficult to insure that the target surface would remain in exactly the same location before and after phase change. The collector-foil holder was placed as close to the target as possible without actually touching it, so that the collector-foil holder could be rotated freely. However, it was impossible to guarantee the relative position of the collector foils to the target surface to better than $0.25 \mathrm{~mm}$. The radius of curvature of the collector-foil surfaces was $33.2 \mathrm{~mm}$. Therefore an error of 28 was introduced by this uncertainty in target-surface position, since the sputtering yield depended upon the square of the distance from the target surface to the collector foils.

\section{Rutherford Backscattering}

The target holder depicted in Figure 6 was mounted on a feedthrough which was at the center of a large scattering chamber. The silicon surface-barrier detector depicted in 
Figure 6 was mounted on an arm which pivoted about this same central point. Consequently, the collector foils which were mounted on the target holder were not at the center of the chamber, and the angle which the detector arm made with the beam trajectory was not the true scattering angle. Each time the Rutherford backscattering arrangement was set up, the distance of the detector aperture from the center of the chamber had to be measured to an accuracy of $\pm 0.03 \mathrm{~mm}$. using a telescoping gauge and a micrometer. Then using this distance and the angle between the detector arm and the central beam trajectory, the true scattering angle was determined. The orientation of the detector aperture also had to be adjusted with a goniometer so that it was truly perpendicular to the trajectory of backscattered particles which traveled from the target holder to the detector. This was the price which had to be paid in order to mount several collector foils simultaneously and thereby avoid the added time involved in repeated pump-downs of the scattering chamber.

In order to determine the solid angle subtended by the detection system, the area of the detector aperture was measured using an optical comparitor. Two detector apertures with areas differing by almost a factor of two were used at different times, and the measured backscattering yields were compared in order to verify that any backscattered particles which passed through the detector aperture were landing in the $50 \mathrm{~mm} .{ }^{2}$ active area of the surface-barrier detector. The ${ }^{19} \mathrm{~F}^{2+}$ ions which were backscattered from gallium or indium 
atoms had far more than sufficient energy to traverse the $40.0 \mathrm{\mu g}$. $\mathrm{cm} .^{-2}$ gold barrier on the detector surface. The least energetic ions of interest were those backscattered from gallium, and they had a range in gold of $2.3 \mathrm{mg} . \mathrm{cm}^{-2}$ Furthermore, the depletion zone in the surface-barrier detector was more than thick enough to stop all the backscattered ions. The range of the ions backscattered off of indium was $2.8 \mu$ in silicon, and the detector depletion depth was $100 \mu$. Consequently, the efficiency of the surface-barrier detector should have been $100 \%$.

The form used for the backscattering differential cross section was:

$$
\left(\frac{\mathrm{d} \sigma}{\mathrm{d} \Omega}\right)_{\mathrm{lab}}=\left(\frac{\mathrm{z}_{1} \mathrm{z}_{2} \mathrm{e}^{2}}{2 \mathrm{E}}\right)^{2} \cdot \frac{\left(\cos \phi+\sqrt{1-\alpha^{-2} \sin ^{2} \phi}\right)^{2}}{\left(\sin ^{4} \phi\right) \sqrt{1-\alpha^{-2} \sin ^{2} \phi}}
$$

$z_{1}$ and $z_{2}$ are the atomic numbers of the incident projectiles and the target atoms, respectively. $E$ is the kinetic energy of the incident projectiles, and $\phi$ is the angle through which they were scattered in the laboratory frame. $\alpha=M_{2} / M_{1}$ where $M_{1}$ and $M_{2}$ are the masses of the incident projectiles and the target atoms, respectively.

The above equation is the laboratory-frame version of the familiar Rutherford cross section. However, this cross section is not valid for all incident ion energies. At low energies, electronic screening of the incident and target nuclei becomes important. At energies where electronic screening is not a factor, nuclear reactions can create reaction products which could be mistakenly counted as 
backscattered particles. The likelihood that electronic screening could be important is eclipsed by the fact that the distance of closest approach for $5.00 \mathrm{MeV}{ }^{19} \mathrm{~F}^{2+}$ on ${ }^{115} \mathrm{In}$ is only $1.48 \times 10^{-11} \mathrm{~cm}$. Meanwhile, the corrected Bohr radius $\left(a_{B} / \sqrt{z_{1}^{2 / 3}+z_{2}^{2 / 3}}\right)$ is $1.26 \times 10^{-9} \mathrm{~cm}$., which is 85 times larger than the distance of closest approach. In order to verify that electronic screening was not a major factor, $10.0 \mathrm{MeV}{ }^{19} \mathrm{~F}^{3+}$ was also used as the incident beam on a collector foil which had collected atoms from a liquid eutectic target. The backscattered yield per incident ion was a factor of four lower than the yield for $5.00 \mathrm{MeV}{ }^{19} \mathrm{~F}^{2+}$, as predicted by the Rutherford cross section. The distance of closest approach for the $10.0 \mathrm{MeV}{ }^{19} \mathrm{~F}^{3+}$ beam was only half as large as the distance of closest approach for the $5.00 \mathrm{MeV}{ }^{19} \mathrm{~F}^{2+}$ beam. As a further check that nuclear-reaction resonances were not contributing spurious counts to the backscattered yield, a $5.1 \mathrm{MeV}{ }^{19} \mathrm{~F}^{2+}$ was also used and yielded results consistent with the $\mathrm{E}^{-2}$ dependence of the Rutherford cross section.

One side of the twelve-sided holder shown in Figure 6 was used to hold a quartz disk so that the ${ }^{19} \mathrm{~F}$ beam spot could be viewed and focussed. The beam spot diameter was $1.5 \mathrm{~mm}$. A $1.5 \mathrm{~mm}$. hole at the bottom of the holder was also used to locate the beam by threading the beam through the hole and minimizing the current on the holder. The quartz viewer was frequently put into position between backscattering runs to verify that the focus and position of the beam had not changed. The possibility that the incident ${ }^{19} \mathrm{~F}^{2+}$ beam was sputtering 
off some of the gallium and indium atoms which had been collected on the graphite foils also needed to be investigated. This was done by taking several long backscattering measurements at the same point on the graphite foil. After a total dose of $5.00 \mathrm{MeV}{ }^{19} \mathrm{~F}^{2+}$ had been accumulated which was five times greater than the average dose normally used, a decrease in backscattered yield of $3 \%$ was observed. Consequently, sputtering during the Rutherford backscattering analysis contributed an error of less than 18 to runs with the average ${ }^{19} \mathrm{~F}^{2+}$ dose.

Figure 6 shows that the current on the target holder was read separately from the current on the electron cage. Typically, the beam current on the target was $250 \mathrm{nA}$ of ${ }^{19} \mathrm{~F}^{2+}$, while the current on the electron cage was $7 \mathrm{nA}$ on the average. Therefore, the beam current integration should have been accurate within $3 \%$. The suppression voltages on the target holder and the electron cage were increased from $\pm 300 \mathrm{~V}$ to $\pm 600 \mathrm{~V}$ with no noticeable change in the target current. This implied that the $\pm 300 \mathrm{~V}$ suppression voltages were sufficient both to repel any electrons which were in the incident beam and to suppress the emission of secondary electrons from the target.

E. Data Analysis

Once a Rutherford backscattering spectrum like the one shown in Figure 13 had been obtained, the peak areas were calculated by simply adding up the total number of counts within 
each peak. Call this total P. The background for each peak was then determined by adding up counts which were to the right and left of each peak. Generally, the number of channels used to determine the high-energy background and the number of channels used to determine the low-energy background were both equal to the number of channels in the peak itself. Call the high-energy background $\mathrm{HB}$ and the low-energy background LB. Then (Bevington, 1969):

$$
\begin{aligned}
\text { area } & =\mathrm{P}-\frac{1}{2}(\mathrm{HB}+\mathrm{LB}) \\
\sigma & =\sqrt{\mathrm{P}+\frac{1}{4}(\mathrm{HB}+\mathrm{LB})}
\end{aligned}
$$

The peak areas were sufficiently large so that $\sigma / a r e a$ was less than 3\%. No correction needed to be made for dead time in counting the pulses which came from the detector since the count rate never exceeded $100 \mathrm{sec}^{-1}$.

After the concentrations of gallium and indium in atoms cm..$^{-2}$ had been determined at various points on the collector foils, these data were combined in order to determine the shape and size of the angular distribution of the sputtered atoms. Data from each foil were given equal weight since the collector system was azimuthally symmetric about the incident $\mathrm{Ar}^{+}$beam. Since the beam spot used for the Rutherford backscattering analysis was only $1.5 \mathrm{~mm}$. in diameter, the cylindrical strips of the collector foils which were analyzed were assumed to be thin slices of a hemisphere of the same radius as the cylinder. Let $d s / d \Omega$ be the differential sputtering yield. The data were fitted to an angular distribution of the form: 


$$
\begin{aligned}
\frac{d S}{d \Omega} & =A \cos ^{B} \theta \\
\ln \frac{d S}{d \Omega} & =\ln A+B \ln (\cos \theta)
\end{aligned}
$$

$\theta$ is the angle with respect to the target normal at which sputtered atoms were ejected. The logarithmic form of the above equation allowed the use of a linear least-squares fit in order to determine the best values of $A$ and $B$ (Bevington, 1969). In order to obtain total sputtering yields, the above angular distribution had to be integrated over $\theta$.

$$
\begin{aligned}
S & =2 \pi \int_{a}^{\pi / 2}\left(A \cos ^{B} \theta\right) \sin \theta d \theta \\
& =2 \pi \frac{A}{B+1}
\end{aligned}
$$

In this chapter, a number of experimental errors have been discussed and estimated: 28 for the beam-current integration during sputtering, 28 for the uncertainty in the position of the target surface, $3 \%$ for the beam current integration during Rutherford backscattering, $1 \%$ due to sputtering during backscattering, and $3 \%$ for the statistical analysis of the Rutherford spectra. The square root of the sum of the squares of these uncertainties results in a total uncertainty of 5\%. Errors such as the uncertainties in the detector solid angle and in the scattering angle during Rutherford backscattering are small compared to the errors listed here. It should be noted that the $5 \%$ total uncertainty is only a rough estimate of the error in determining sputtering 
yields. An alternative error estimate could be made by using $\sigma_{A}$ and $\sigma_{B}$, the standard deviations of the parameters $A$ and $B$ mentioned above. However, not all of the uncertainties listed here would manifest themselves in $\sigma_{A}$ or $\sigma_{B}$. Furthermore, some of the uncertainties are of a systematic nature and would not be given sufficient weight in an estimate of only random errors. It should also be noted that $A$ and $B$ are heavily correlated. For example, if the incident sputtering beam is not perfectly well aligned with the collector-foil holder; the angular distribution will be skewed, and a large error in $B$ will result. However, $A /(B+1)$ will be left unchanged. 


\section{RESULTS}

A. Elemental Gallium and Indium

Table 2 summarizes the results of sputtering pure gallium and pure indium targets. Two sputtering runs of pure solid gallium were conducted where the only significant difference between the runs was that one had twice as much $\mathrm{Ar}^{+}$dose as the other. The two sputtering yields for these runs agree within the experimental uncertainty, which indicates that the sputtering yield is dose independent. The dose independence of the sputtering yield implies two things. First of all, the cleanliness of the target surface is not changing during the sputtering. Secondly, the sticking probability of sputtered gallium atoms on the collector foils is not changing as gallium accumulates on the surface of the foils.

The angular distribution of the low-dose and high-dose solid gallium runs are shown in Figures 7 and 8 , respectively. As Table 2 indicates, the angular distributions are much sharper than $\cos \theta$. Similar sharp distributions were observed by Emmoth and Braun when they sputtered a silver target with $\mathrm{Ar}^{+}$ions ranging in energy from $15 \mathrm{keV}$ to $80 \mathrm{keV}$ (Emmoth and Braun, 1977). At $15 \mathrm{keV}$, the angular distribution of sputtered silver followed a $\cos ^{2} \theta$ function. As the energy of the incident $\mathrm{Ar}^{+}$ions was increased to $80 \mathrm{keV}$, the angular distribution flattened out but still did not approach a $\cos \theta$ dependence. Apparently, the angular distribution is heavily dependent on target material since Emmoth observed a $\cos \theta$ 
distribution for atoms sputtered from a tungsten target for the whole range of incident $\mathrm{Ar}^{+}$energies from $15 \mathrm{keV}$ to 80 kev. Rödelsperger observed $\cos ^{2} \theta$ distributions for $130 \mathrm{keV}$ $\mathrm{Ar}^{+}$on five different elemental targets (Rödelsperger and Scharmann, 1976). Here the incident beam was not normal to the target surface but came in at $60^{\circ}$ to the target normal. Furthermore, several sharp angular distributions have been observed in the sputtering of alloys. For example, H. H. Andersen obtained a near $\cos ^{2} \theta$ dependence for copper atoms sputtered from a copper-platinum alloy by $80 \mathrm{keV} \mathrm{Ar}^{+}$(Andersen et al., 19812.

The possibility that the sharp angular distributions may somehow be caused by facetting of the target surface is rendered implausible by the fact that the liquid gallium targets also have sharp angular distributions. In fact, the angular distributions from the liquid gallium targets are $\cos ^{2} \theta$ within experimental error. Figures 9 and 10 depict two angular distributions taken from liquid gallium targets. As shown in Table 2, one of the liquid gallium runs was the first run conducted in this experiment and had incorrect beam current integration. Therefore, no sputtering yield is listed for this run; but an angular distribution is included since only relative yields are needed for that.

The only previous measurement of an angular distribution from a liquid target was made by H. L. Garvin (Garvin, 1968). The target was liquid aluminum. First the distribution of vaporized aluminum was measured and found to follow a $\cos \theta$ 
function, as expected. Then the distribution of aluminum atoms sputtered from liquid aluminum by $5 \mathrm{keV} \mathrm{Hg}^{+}$was measured. The six data points taken lie very close to a $\cos ^{2} \theta$ function.

Although the sputtering yield for liquid gallium appears slightly higher than the yield for the solid, the difference is not inconsistent with the experimental uncertainty. Unfortunately, H. I. Garvin sputtered only liquid gallium, so there are no results with which to compare. As stated earlier, Garvin measured only relative sputtering yields. However, Garvin did find the gallium-ion sputtering yield versus target temperature to be constant within $20 \%$ until the gallium was heated above $800^{\circ} \mathrm{C}$ and evaporation started to play a role. No measurements were made of the sputtering of neutral gallium atoms.

The sputtering of solid and liquid indium is also summarized in Table 2. Once again both solid and liquid show sharp angular distributions, and the liquid distribution is consistent with $\cos ^{2} \theta$. The angular distribution for solid indium is shown in Figure 11, and the distribution for liquid indium is in Figure 12. The points in the solid indium distribution show considerably more scatter than in the liquid indium distribution, which could be due to surface irregularities in the solid. The sputtering yield for liquid indium is lower than the yield for solid indium, although again the difference is within experimental uncertainty. Garvin observed a $14 \%$ decrease in the yield of indium ions sputtered by $6 \mathrm{keV}$ 
$\mathrm{Hg}^{+}$when the indium target was melted. No effect of that size has been observed here.

It should be noted that although the solid angular distributions were not quite consistent with $\cos ^{2} \theta$, they were very close. Furthermore, the differences in angular distribution for solid and liquid elemental targets were all within experimental error.

B. Liquid Gallium-Indium Eutectic Alloy

The results of sputtering the liquid gallium-indium eutectic alloy are summarized in Table 3 . One of the runs was actually conducted below the melting point of the eutectic. However, the target was still liquid due to the supercritical behavior mentioned earlier. The eutectic alloy is $83.5 \%$ gallium and $16.5 \%$ indium (atomic $\%$ ). However, the table indicates that the sputtered material contained 5.6 times as many indium atoms as gallium atoms for the $15 \mathrm{keV} \mathrm{Ar}^{+}$runs. (Note that the $\mathrm{Ar}^{+}$energy is listed as $14.7 \mathrm{keV}$ because of the electron suppression on the target.) The sputtering yields for the two $15 \mathrm{keV}$ runs agree with each other within the experimental uncertainty. A typical Rutherford backscattering spectrum taken from a collector foil which was used over a liquid eutectic target is shown in Figure 13. The Rutherford cross section for ${ }^{19} \mathrm{~F}$ backscattering from indium is 2.5 times greater than the cross section for backscattering from gallium.

Not only is the partial sputtering yield for indium much greater than the gallium yield, but the angular distributions 
for gallium and indium are also quite different. The angular distributions for gallium and indium sputtered from the liquid eutectic by $15 \mathrm{keV} \mathrm{Ar}^{+}$are shown in Figures 14 and 15, respectively. As the figures readily show, the gallium distribution is much sharper than the indium distribution. The angular distributions for the two $15 \mathrm{keV}$ liquid eutectic runs agree with each other within experimental error as shown in Table 3. Indium follows $a \cos ^{2} \theta$ distribution, while the gallium distribution is $\cos ^{3} \cdot 6 \theta$.

The results from the liquid eutectic sputtering were unexpected. First of all, the ratio of the indium yield to the gallium yield for the $15 \mathrm{keV} \mathrm{Ar}^{+}$is 28 times higher than would be expected from the target stoichiometry. Furthermore, the fact that indium is sputtered more readily than gallium defies both theories of surface segregation which were summarized in the introduction. One theory predicts that the lighter atom (in this case, gallium) should be ejected preferentially. The other theory predicts that the atom which is most abundant in the target should be sputtered preferentially. Both theories state that the concentration of gallium in the sputtered material should be higher than the gallium concentration in the target.

The possibility of evaporation causing the high indium yield is implausible in light of the fact that the target temperature would have to be $600^{\circ} \mathrm{C}$ before indium and gallium would be evaporated in a ratio equal to the observed sputtering yield ratio. When the power of the incident beam 
and the thermal conductivity of the target are considered, the difference between the target temperature at the beam spot and the target temperature at the rim of the target can be no more than $0.03^{\circ} \mathrm{C}$. (Recall the power which the beam dumps into the target is only $9 \mathrm{~mW} .1$ of course, this calculation says nothing about the possibility of thermal spike sputtering. In order to address this question, we refer again to the fact that Garvin did not see an upturn in the gallium sputtering yield until the target temperature exceeded $800^{\circ} \mathrm{C}$. This was the same temperature required to vaporize the gallium with no incident radiation. Consequently, the $6 \mathrm{keV} \mathrm{Hg}^{+}$beam which Garvin used showed no signs of setting up thermal spikes in the liquid gallium target. If there had been a thermal spike effect, the temperature at which the sputtering yield increased would have been lower than $800^{\circ} \mathrm{C}$. Now the temperature of a thermal spike is proportional to the energy of primary recoil atoms in the target (Nelson, 1965), and the maximum primary recoil energy is:

$$
E_{r}=E \frac{4 m M}{(m+M)^{2}}
$$

$\mathrm{E}$ and $\mathrm{m}$ are the energy and mass of the incident projectiles, and $M$ is the mass of the target atoms. Thus the primary recoil energy for gallium recoils from $15 \mathrm{keV} \mathrm{Ar}^{+}$is a factor of three greater than the energy for recoils from $6 \mathrm{keV} \mathrm{Hg}{ }^{+}$. So the temperature of any thermal spikes which could occur in our experiment should be only a factor of three greater than the thermal spike temperature in Garvin's experiment, 
which we have already established to be negligible. (Note that Garvin's temperature readings were accurate to within $15^{\circ} \mathrm{C} .1$ By the way, even if thermal spike sputtering were to occur, all extant theories would predict equipartition of energy between the target atoms and preferential sputtering of the lighter atoms (Sigmund, 1980).

The fact that the gallium angular distribution is much narrower than the indium distribution might at first glance suggest that reflective collisions are going on inside the target. The phenomenon of reflective collisions occurs when an incident ion strikes one of the lighter target atoms, thereby creating a primary recoil atom. This light recoil atom then collides with a heavy target atom, backscatters from the heavy atom, and is ejected from the target. A similar process cannot occur for the heavy primary recoil atoms since heavy atoms cannot backscatter from lighter atoms or even other heavy atoms of the same mass. Reflective collisions result in a narrow angular distribution for the lighter sputtered atoms. However, reflective collisions are probably not a factor in this experiment for two reasons. First of all, reflective-collision sputtering can take place only when the primary recoil occurs close to the surface. Since the range of $15 \mathrm{keV} \mathrm{Ar}^{+}$in gallium is approximately $300 \AA$, the primary recoil will occur too deep inside the target for the recoil atom to backscatter and still make it out of the target. Instead, the recoil atom will give up its energy to many other target atoms in a long succession 
of collisions. Note that reflective collision effects have not been observed for incident $\mathrm{Ar}^{+}$energies greater than $1 \mathrm{keV}$ (Olson et al., 1979). The second reason why reflective collisions are probably not important is that reflectivecollision theory does not predict a preferential sputtering of the heavier atoms that we have observed.

One possible explanation for the observed results is that the liquid eutectic target may have a segregated surface before we even begin sputtering. If the surface were heavily enriched in indium (even though indium is more dense than gallium), indium atoms would be sputtered preferentially since atoms on the surface should be ejected more easily than atoms from inside the target. Furthermore, if the sputtered gallium atoms have to come from beneath the indium-enriched surface, they might not have enough energy to traverse the surface at angles which are nearly parallel to the plane of the surface. The shortest way for these atoms to get out would be to follow a trajectory normal to the target surface, and this could explain why the gallium distribution is so narrow. Note that in order to maintain the hypothetical indium-enriched surface, indium atoms would have to diffuse from the bulk of the target to the surface at a rate equal to their sputtering rate, $i$. e., approximately one monolayer every 10 seconds.

As a step toward testing the above hypothesis, the liquid eutectic target was sputtered with $25 \mathrm{keV} \mathrm{Ar}^{+}$(the highest energy beam the ion source was capable of producing) 
to see if a higher energy beam would impart more energy to the atoms beneath the surface and allow them to escape more easily. As Table 3 shows, the ratio of indium to gallium did decrease to 2.6. Furthermore, the partial sputtering yield of gallium increased by $48 \%$; and the angular distribution of gallium broadened to a $\cos ^{2} \theta$ function. There was no noticeable change in the indium distribution, i. e., it remained $\cos ^{2} \theta$. The angular distributions for gallium and indium sputtered from the liquid eutectic by $25 \mathrm{keV} \mathrm{Ar}^{+}$are depicted in Figures 16 and 17, respectively.

C. Solid Gallium-Indium Eutectic Alloy

Although indium and gallium atoms are miscible in the liquid phase, they are almost completely immiscible in the solid phase. The solid solubility of indium in gallium is less that 0.3 atomic per cent (Hansen, 1958). This means that when the eutectic alloy was frozen, separate gallium and indium crystallites formed. The results of sputtering such a collection of crystallites are summarized in Table 4. The dashed line separates data taken from two targets which were frozen under different conditions. The first target was frozen gradually in the UHV target chamber by putting a dry ice pack on the target-assembly flange as mentioned earlier. The second target was frozen in liquid nitrogen outside the target chamber, quickly put in place inside the chamber, and cooled with an ice pack immediately after roughing had begun before the target had a chance to melt. 
One aspect of the sputtering of the solid eutectic which is quickly apparent is that the composition of the sputtered material is highly dependent upon dose. Figures 18, 19, and 20 show Rutherford backscattering spectra of collector foils which represent sputtering from progressively deeper slices of the second frozen target. At first, the sputtered material is predominantly indium; but after the target has been bombarded with more $\mathrm{Ar}^{+}$and more of the target has been sputtered away, the sputtered material is predominantly gallium. Note the similarity between Figure 18 which represents the initial sputtering of the solid eutectic and Figure 13 which represents the sputtering of the liquid eutectic. The similarity cannot be due to localized melting of the solid eutectic since the run in Table 4 which was done with the highest target temperature $\left(11.6^{\circ} \mathrm{C}\right)$ shows a predominantly gallium yield, unlike liquid eutectic sputtering. Since the composition of the sputtered material is changing with dose, the yields and distributions recorded in Table 4 are really averages over ranges of incident $\mathrm{Ar}^{+}$ dose. For that reason, the total amount of $\mathrm{Ar}^{+}$accumulated before the beginning of and at the end of each run is recorded. Figure 21 shows the composition of the sputtered material versus incident $\mathrm{Ar}^{+}$dose. The indium concentration in atomic per cent is determined from Table 4 . The shaded region represents the quickly frozen target while the unshaded blocks represent the target which was frozen gradually. A short sputter-cleaning run was conducted on the slowly frozen target 
which did not sputter off enough gallium to provide good statistics for an angular distribution. However, the regions of the collector foils which corresponded to small values of $\theta$ did have enough atoms to make an estimate of the sputtered composition. This estimate of $96 \%$ indium is the block which is farthest to the left in the histogram shown in Figure 2l, and it probably underestimates the indium concentration since the gallium angular distributions are typically sharper than the indium distributions.

As Figure 21 shows, the sputtered composition from both frozen targets eventually reaches the bulk eutectic composition. However, the quickly frozen target reaches the bulk composition with less incident $\mathrm{Ar}^{+}$dose than the slowly frozen target. Both targets show a predominance of indium crystallites on the surface which have to be sputtered away before the bulk composition can be reached. This fact further suggests the possibility that the surface of the liquid eutectic is predominantly indium because an indium-enriched surface could act as a "seed" surface on which indium crystallites could form upon freezing. The quickly frozen target has smaller indium crystallites on its surface than the slowly frozen target since it requires less incident $\mathrm{Ar}^{+}$to sputter them away. However, even the quickly frozen target has to have approximately 2000 monolayers removed before the bulk composition is reached. It should be noted that these effects are of a very gross nature compared to observations of surface segregation in well-mixed binary solids where the bulk 
composition is reached after removal of a few hundred monolayers and where one species is enriched on the surface by at most 308 (Liau et al., 1978).

one further trend which can be extracted from Table 4 is that as more and more target material is sputtered away, the angular distribution for indium becomes broader. Figures 22 and 23 indicate the broadening of the indium distribution for the target which was frozen gradually. Figures 24 through 26 show the broadening of the indium distribution for the quickly frozen target. As we go from the surface which is dominated by indium crystallites approximately 2000 monolayers in diameter down into the bulk of the target, the average size of the indium crystallites should decrease. This decrease in the average size of the crystallites could broaden the angular distribution in much the same was as surface roughness can.

Caution should be exercised in trying to extract too much information from the solid eutectic sputtering since the solid eutectic targets are really inhomogeneous collections of crystallites. The size of the crystallites and the way in which they are packed together depend heavily upon the history of the conditions under which the target was frozen. Although it was stated in the introduction that sputtering yields should be independent of target density, this independence applies only to targets of homogeneous density. Discontinuities in the density caused by spaces or gaps in the target definitely would affect the sputtering yield. 
D. Ion Scattering Spectroscopy

In order to approximately determine the composition of the surface of the liquid eutectic, a brief Auger analysis was conducted at the Rockwell International Corporation Science Center in Thousand Oaks, California. No standards were made for the analysis. Approximate "textbook" standards were used instead, and therefore the results had an accuracy of no better than 20\%. The Auger analysis yielded a composition which was equal to the bulk eutectic composition and did not indicate indium enrichment. However, the Auger analysis examined a surface depth of 5 or 6 monolayers, and therefore the results were averaged over this depth. An ESCA analysis of the liquid eutectic resulted in a surface concentration of 368 for indium. This is clearly an indium enrichment, but the uncertainty in the measurement is difficult to estimate since the standards which were used were oxidized. The ESCA result represented an average over approximately 3 or 4 monolayers. It should also be noted that the ESCA spectrum for the liquid eutectic showed no signs of implanted argon from the $3 \mathrm{keV} \mathrm{Ar}{ }^{+}$used for sputter-cleaning. When the eutectic was solidified, the ESCA easily detected the predominance of indium crystallites on the surface.

The above data motivated us to seek a surface analysis technique which would be sensitive to only the first monolayer of the surface so that the surface composition could be determined without averaging over several monolayers of the bulk composition. Ion scattering spectroscopy is such a 
technique. When noble gas ions of energies of a few kev or less strike a surface, the ions which go beneath the first monolayer are neutralized. The ions which backscatter from the target as ions are predominantly those which backscatter from atoms in the first monolayer of the surface (Smith, 1971; Taglauer and Heiland, 1976).

An ISS apparatus was designed, constructed, and placed in the sputtering apparatus between the analyzing magnet and the target chamber. (See Figure 27.) The accelerator produced $15 \mathrm{keV} \mathrm{Ar}^{+}$which was used for sputter cleaning as well as $2 \mathrm{keV} \mathrm{Ar}^{+}$which was used for the ISS. Since the maximum beam current fell off rapidly with a decrease in energy, only $30 \mathrm{nA}$ of $2 \mathrm{keV} \mathrm{Ar}{ }^{+}$could be obtained. Some details of the construction of the ISS apparatus are included in the Appendix.

All ISS scattering was done with a $90^{\circ}$ scattering angle. Let $\mathrm{E}$ be the incident ion energy and $\mathrm{E}_{\mathbf{S}}$ the energy of the backscattered ions. Also let $\mathrm{m}$ and $\mathrm{M}$ be the masses of the incident projectiles and the target atoms, respectively. Then:

$$
\frac{E_{S}}{E}=\frac{M-m}{M+m}
$$

Figures 28 and 29 are ISS spectra taken from a solid gallium target and a solid indium target, respectively. Note that there are large low-energy backgrounds in these spectra which are characteristic of ISS spectra and which are thought to be due to sputtered ions as well as some $\mathrm{Ar}^{+}$ions 
which have been backscattered from beneath the first monolayer. The reproducibility of the peak areas in these spectra was no better than $20 \%$ because the target was unbiased so that the energies of the backscattered ions would not be disturbed. The unbiased target resulted in beam current integrations which were not reproducible to better than 20\%. The ratio of the indium peak area to the gallium peak area was $2.0 \pm 0.5$. We expected a ratio somewhere between 1 and 2.5 since the cross section is assumed to be a screened-collision cross section.

Finally, a liquid eutectic target was made by painting the alloy on a tantalum backing. A large amount of liquid could not be supported because the target had to be held at $45^{\circ}$ with respect to the incident vertical beam. The ISS spectrum for the liquid eutectic is depicted in Figure 30 . A small tantalum peak appeared because the sputter-cleaning run actually exposed some of the tantalum backing, which was discovered when the target was removed. As Figure 30 shows, we cannot be certain that there is any real gallium peak at all. The peak areas from Figure 30 and the above sensitivity factor imply that the gallium concentration at the first monolayer of the liquid eutectic must be less than 6\%. Recall that the bulk composition is $83.5 \%$ gallium. 


\section{DISCUSSION}

With the exception of the sharp gallium angular distribution observed for the $15 \mathrm{keV} \mathrm{Ar}^{+}$sputtering of the liquid eutectic target, all of the angular distributions for atoms sputtered from liquid targets were consistent with $\cos ^{2} \theta$. However, theory predicts a $\cos \theta$ distribution (Sigmund, 1980). While no attempt will be made to reproduce collision-cascade theory, that part of the theory which produces the $\cos \theta$ can readily be discussed.

After the incident projectile enters the target and produces a primary recoil, the primary recoil atom will share its energy with a number of secondary recoil atoms inside the target. A whole collision cascade of these secondary recoils is established. Some of these secondaries will cross the target surface boundary and become sputtered atoms. However, they do not cross the boundary with a straight-line trajectory. Instead they are refracted, and it is the nature of this refraction which produces the angular distribution of the sputtered material. Let $\theta^{\prime}$ be the angle which the trajectory of the secondary atom makes with the surface normal immediately before the atom crosses the surface. Let $\theta$ be the angle of the trajectory of the atom after ejection. Similarly, let $\varepsilon^{\prime}$ and $\varepsilon$ represent the kinetic energy of the atom immediately before and after ejection, respectively. Call $\mathrm{U}$ the surface binding energy. Then:

$$
\varepsilon=\varepsilon^{\prime}-\mathrm{U}
$$




$$
\begin{aligned}
\cos \theta & =\sqrt{(1+U / \varepsilon) \cos ^{2} \theta^{\prime}-U / \varepsilon} \\
& =\sqrt{\frac{\cos ^{2} \theta^{\prime}-U / \varepsilon^{\prime}}{1-U / \varepsilon^{\prime}}}
\end{aligned}
$$

The above refraction relation assumes that the component of the atom's momentum which is parallel to the surface remains constant as the atom crosses the surface boundary. However, some of this momentum component can be lost at the surface, especially if the atom is ejected nearly parallel to the surface. Such a loss would tend to sharpen the angular distribution. Furthermore, in using the above refraction relation most theories assume that $U$ is a constant. In other words, all atoms are supposed to lose exactly the same amount of energy regardless of the angle at which they cross the surface. But if $U$ were a function of $\theta^{\prime}$, the angular distribution could be either broadened or sharpened depending on how $U$ varies with $\theta^{\prime}$. In addition to the sharp distributions which were cited in the previous chapter, several angular distributions which are broader than $\cos \theta$ have also been observed for incident ion energies of less than $1 \mathrm{keV}$ (Wehner et al., 1960).

P. Sigmund had predicted in his most recent theoretical work that atoms of one kind which are sputtered from beneath a surface-enriched layer of another kind of atom should manifest a sharp angular distribution (Sigmund, 1981). This agrees qualitatively with the gallium distribution for the $15 \mathrm{keV}$ sputtering of the liquid eutectic. However, the angular 
distribution which sigmund derives is not expressible as a simple power of $\cos \theta$. Furthermore, Sigmund's distribution has no explicit dependence on the energy of the incident projectiles. Recall that the angular distribution of gallium broadened from $\cos ^{3.6} \theta$ to a $\cos ^{2} \theta$ distribution when the incident $\mathrm{Ar}^{+}$energy was increased from $15 \mathrm{keV}$ to $25 \mathrm{keV}$. It is interesting to note that Russell observed a surface enrichment of ${ }^{44} \mathrm{Ca}$ and an angular distribution which showed a higher fraction of ${ }^{4}{ }^{\circ} \mathrm{Ca}$ at $5^{\circ}$ to $25^{\circ}$ than at $41^{\circ}$ to $72^{\circ}$ (Russell et al., 1980).

Although evidence was presented in the previous chapter for the existence of an indium-enriched monolayer on the surface of the liquid gallium-indium eutectic, no reason was given as to why such an enriched layer should be formed. Consequently, the thermodynamics of the eutectic surface needs to be explored.

Recent theoretical work has related the energy of a metallic surface to the properties of the atoms which comprise the surface (Miedema, 1978). The surface energy (the atomic analog of surface tension) is determined by considering both the electron work function and the electron density of an atomic cell. In general, the higher the work function, the lower the surface energy. This is because atomic cells with high work functions find it energetically unfavorable to share their electrons with other cells. Consequently, thermodynamics favors the presence of such cells at the surface where there is an entire half-space with no nearest 
neighbors. It is also a general rule that atomic cells with low electron densities should also have low surface energies. Atoms at the surface are required to alter their pure-metal electron densities in order to match electronic wave functions with the vacuum where the wave functions have to be zero. Such an alteration can occur more easily for atomic cells which have low electron densities to begin with. Let $\gamma$ represent the surface energy, and let $\phi$ represent the electron work function. Furthermore, let $\mathrm{n}$ be the electron density at the boundary of the atomic cell. Based upon considerations which have only been touched upon here, A. R. Miedema arrived at a semi-empirical form for the surface energy:

$$
\gamma=3176 \frac{\mathrm{n}^{5 / 3}}{(\phi-0.6)^{2}} \frac{\operatorname{erg~} \mathrm{eV}^{2}}{\mathrm{~cm}^{2} \text { (density units) }^{5 / 3}}
$$

When appropriate values for $\phi$ and $n$ are used (Miedema, 1976), we find $\gamma=1000$ ergs $\mathrm{cm} .^{-2}$ for gallium and 640 ergs $\mathrm{cm} .^{-2}$ for indium. Since indium has the lower surface energy, the indium concentration should be enriched at the surface.

We would like to estimate the size of the predicted indium enrichment. In order to do this, we first need to know the change in entropy $(\Delta S)$ when a bulk indium atom exchanges positions with a gallium atom at the surface in order to reach a surface equilibrium. Let $\mathrm{x}_{\mathbf{S}}$ be the indium surface concentration at equilibrium. Let $\mathrm{x}_{\mathrm{b}}$ be the bulk indium concentration. Then (Lambin et al., 1980):

$$
\Delta S=-k \text { ln }\left[\frac{x_{s}\left(1-x_{b}\right)}{\left(1-x_{s}\right) x_{b}}\right]
$$


$\mathrm{k}$ is Boltzmann's constant. Note that if $\mathrm{x}_{\mathrm{s}}=\mathrm{x}_{\mathrm{b}}$, then $\Delta s=0$. In order for the Gibbs free energy to be zero, we must set;

$$
\Delta \gamma=\gamma_{\text {In }}-\gamma_{\mathrm{Ga}}=\mathrm{n}(\mathrm{T} \Delta \mathrm{S})
$$

$\mathrm{n}$ is the average number of atoms per $\mathrm{cm} .^{2}$ in a monolayer, which is needed in order to match the units in which $\gamma$ is normally expressed. $T$ is the absolute temperature. When the expression for $\Delta S$ is placed in the above equation, we obtain (Hamilton, 1979):

$$
\frac{x_{s}}{1-x_{s}}=\frac{x_{b}}{1-x_{b}} \exp \left[\frac{-\left(\gamma_{I n}-\gamma_{G a}\right)}{n k T}\right]
$$

Thus $\mathrm{x}_{\mathrm{s}}=0.995$, and this theory of surface segregation predicts a surface monolayer of almost pure indium.

The above theory has been used by J. C. Hamilton to successfully predict surface segregation in 35 binary alloys for which surface composition measurements have been made. It should be noted that Hamilton only predicted whether or not segregation would occur and which species should be enriched at the surface. No estimates of the size of the enrichment were mentioned. Furthermore, most of the surface segregations were observed by Auger analysis which usually had a depth resolution of no better than 5 monolayers. However, one measurement done on copper-nickel alloys achieved better depth resolution by performing a careful analysis of the mean free paths of the Auger electrons (Watanabe et al., 1976). This measurement showed that the observed surface segregation was confined almost totally to the first monolayer. 
Nothing in the above theory would imply segregation any deeper than the first monolayer.

The ISS analysis of the liquid eutectic established an indium concentration in the first monolayer of $0.97 \pm 0.03$. (Recall the upper limit on the gallium concentration was 68.2 In order for this result to be consistent with the ESCA measurement of 368 indium, the indium enrichment must be confined to the first monolayer. Let $\mathrm{x}$ represent the fraction of sputtered atoms which come from the first monolayer of the target surface. Then $(I-x)$ is the fraction of sputtered atoms which originate from beneath the first monolayer. Let $f$ represent the fraction of the sputtered material which is indium. If we assume that the sputtered material which comes from beneath the surface is representative of the bulk composition, then:

$$
\begin{aligned}
& f=0.97 x+0.165(1-x) \\
& x=\frac{f-0.165}{0.805}
\end{aligned}
$$

$f$ can be determined from Table 3 for the sputtering of the liquid gallium-indium eutectic. Thus:

$$
\begin{array}{rlrl}
x & =0.85 \pm 0.03 & & \text { for both } 15 \mathrm{keV} \mathrm{Ar} \\
& =0.70 \pm 0.03 & & \text { for the } 25 \mathrm{keV} \mathrm{Ar} \\
+ & \text { run }
\end{array}
$$

The errors in the above values for $x$ are due to the uncertainty in the precise composition of the first monolayer. The error in $f$ is relatively small since any systematic errors in the measurement of the sputtering yields should cancel out in determining $f$. 
It has always been assumed that most sputtered atoms come from the target surface since most sputtered atoms have low kinetic energies (a few ev). To the best of our knowledge, this is the first time that the fraction of sputtered atoms which come from the first monolayer has been quantified. Because of its sensitivity to the composition of the first monolayer, the sputtering of liquids can play a role in the field of surface analysis, especially where a very narrow depth resolution is required. It may also be an important factor in attempts to make amorphous alloys on surfaces by melting the surfaces with incident radiation. However, the effects observed here would be overridden if the intensity of the incident radiation were high enough to cause evaporation (Krutenat and Gesick, 1970).

A final word should be said about the formation of indium crystallites on the surface of the solid eutectic. Although we suggested that the formation might have something to do with the observed indium layer on the liquid eutectic, other explanations could be given which are based upon the metallurgy of the target. One could consider the possibility that the alloy we used might have had slightly too much indium in it to be the true eutectic alloy. It could be further hypothesized that the atoms in the target would like to crystallize according to the true eutectic composition, and that some indium was simply left over at the surface. This particular explanation turns out to be unlikely when we consider that the amount of indium which was observed to be 
on the surface represents only a very small fraction of the total indium in the target. If the above explanation were correct, it would imply that the indium concentration in the target was too high by $0.003 \%$ which is far smaller than the uncertainty in our knowledge of what the eutectic composition really is. 
Appendix - Ion Scattering Spectroscopy Apparatus

Figure 31 illustrates the apparatus which was built in order to analyze and detect low-energy ions which are scattered from the target at $90^{\circ}$ with respect to the incident vertical ion trajectory. The surface of the target makes a $4^{\circ}$ angle with the plane of the diagram and is positioned to intercept the vertical ion beam. In order to be detected, the scattered ions must first pass through the $0.15 \mathrm{~mm}$. aperture which is located at the entrance of the electrostatic analyzer. This aperture is grounded, and its width determines the energy resolution of the analyzer which is $0.5 \%$. As Figures 28 through 30 show, the peak widths in the Iss spectra are considerably larger than the analyzer resolution alone would imply. However, the energy resolution of the incident beam, the nonzero beam spot diameter, and inelastic scattering also contribute to the peak widths. The two cylindrical segments which comprise the electrostatic analyzer are held at opposite voltages so that the central path through the analyzer is at ground potential. After the ions which have the appropriate energy traverse the analyzer $\left(E_{S}=10 \mathrm{x}\right.$, $_{\text {, }}$ where $\mathrm{V}$ is the analyzer voltage), they strike the entrance of the channeltron detector which is biased at $-2800 \mathrm{v}$. The voltage at the entrance of the channeltron serves to accelerate the incident positive ions as well as to drive the electron cascade which makes the detector work. The channeltron produces a pulse of approximately $10^{8}$ electrons 
for each ion which is incident at its entrance cone. These pulses are collected on an anode plate which is biased at $+300 \mathrm{~V}$. The real-life version of Figure 31 is shown in Figure 32.

Figure 33 is a schematic of how the electronics were set up. The beam current on target is fed into a current digitizer which produces a pulse for every $10^{-7}$ Coulomb of integrated charge. The pulse from the digitizer is in turn fed into the multichannel analyzer and instructs the multichannel to advance to the next channel。 As the multichannel advances, the $\mathrm{x}$-axis display output increases in voltage. This output is amplified in order to provide the voltages for the electrostatic analyzer. So when the multichannel advances one channel, the electrostatic analyzer is set to analyze for a correspondingly higher energy. When the electrostatic analyzer voltage is plotted versus the total accumulated charge on target, a staircase results. The rest of Figure 33 is devoted to pulse handling electronics for the pulses which come out of the channeltron detector. The entire process described above is an example of multichannel scaling。

Figure 34 is the amplifier which was designed to amplify the $\mathrm{x}$-axis display output from the multichannel analyzer in order to provide positive and negative voltages for the electrostatic analyzer. First of all, the x-output is added to an offset voltage since the range of the $x$-output is normally $-1 \mathrm{~V}$ to $+1 \mathrm{~V}$. The addition is carried out through an inverting amplifier with adjustable gain. The output of this amplifier 
is fed into a non-inverting amplifier in order to obtain the negative voltage for the electrostatic amplifier. The positive voltage is obtained from the upper part of the circuit which is in a sense a mirror image of the lower part of the circuit. The response time of this circuit is very high ( $0.4 \mathrm{msec})$ ) but since the average time per channel is 3 sec., the response time does not pose a problem. The output of the circuit shows some $60 \mathrm{~Hz}$ noise, but the amplitude of the noise is less than $0.05 \mathrm{~V}$ for a $100 \mathrm{~V}$ output. 
REFERENCES

Andersen, H. H., Chevellier, J. and Chernysh, V., 1981, "The Angular Distribution of Material Sputtered from AgAu and CuPt by 20-80-keV Argon," to be published.

Bevington, P. R., 1969, Data Reduction and Data Analysis for the Physical Sciences (McGraw-Hill, New York), Pp. 92-118.

Cochran, C. N. and Foster, I. M., 1962, J. Electrochem. Soc。 $109,144$.

De la Breteque, Pierre, 1962, Gallium: Principal Properties and Bibliography (Aluminum Industrie Aktien Gesellschaft, Marseille), pp. 1-25.

Emmoth, B. and Braun, M., 1977, Proc。7th Intern. Vac. Congr. \& 3rd Intern. Conf. Solid Surfaces, Vienna, 1465.

Gabta, L., 1981, "Photon Emission from Sputtered Atoms as a Function of the Phase Transitions in the Targets," paper presented at the Ninth International Conference on Atomic Collisions in Solids, Lyon, July 6-10, 1981. Proceedings to be published in Nuclear Instruments and Methods.

Garvin, H. L., 1968, "Measurement of Sputtering Yields and Energy Transfer Efficiencies of Liquid Metals," NASA, report \#CR-54678.

Haff, P. K., 1977, Appl. Phys. Lett. 31, 259.

Haff, P. K. and Watson, C. C., 1979, J. Geophys. Res. 84, 8436. Hamilton, J. C., 1979, Phys. Rev. Lett. $\underline{42}$, 989.

Hansen, Max, 1958, Constitution of Binary Alloys (McGraw-Hill, New York), pp. 745-746.

Krutenat, R. C. and Panzera, C., 1970, J. Appl. Phys. 4l, 4953.

Krutenat, R. C. and Gesick, W. R., 1970, J. Vac. Sci. Technol. 7. 540 .

Lambin, P。 and Gaspard, J. P., 1980, J. Phys. F: Metal Phys. 10,2413 .

Liau, Z. L., Mayer, J. W., Brown, W. L. and Poate, J。 M., 1978, J. Appl. Phys. 49, 5295.

Miedema, A. R., 1976, Plutonium 1975 and Other Actinides, edited by $\mathrm{H}$. Blank and R. Linder (North-Holland, Amsterdam), p. 6 。 
Miedema, A. R., 1978, Z. Metalkd. 69, 287.

Nelson, R. S., 1965, Phil. Mag. 11, 291。

Nesmeyanov, Andrei N., 1963, Vapour Pressures of the Elements, translated and edited by J. I. carasso (Academic Press, New York), pp. 444, 450-459.

Olson, R. R., King, M. E. and Wehner, G. K., 1979, J. Appl. Phys. 50,3677 .

Rödelsperger, K. and Scharmann, A。, 1976, Nuc. Instr. \& Meth. $132,355$.

Russell, W. A., Papanastassiou, D. A. and Tombrello, T. A., 1980, Radiation Effects 52, 41。

Sigmund, Peter, 1980, "Sputtering by Ion Bombardment: Theoretical Concepts," preliminary version of chapter 2 of Sputtering by Ion Bombardment, R. Behrisch, editor, to be published.

Sigmund, P., Olivia, A., and Falcone, G., 1981, "Sputtering of Multicomponent Materials: Elements of a Theory," paper presented at the Ninth International Conference on Atomic Collisions in Solids, Lyon, July 6-10, 1981. Proceedings to be published in Nuclear Instruments and Methods.

Smith, David P., 1971, Surface Science 25, 171.

Taglauer, E. and Heiland, W., 1976, Appl. Phys. 9, 261.

Watanabe, K., Hashiba, M. and Yamashina, T., 1976, Surface Science $61,483$.

Watson, C. C. and Haff, P. K., 1980, J. Appl. Phys. 51, 691. Wehner, G. K. and Rosenberg, D., 1960, J. Appl. Phys. 31, 177. 
Table 1

Results of the collector-foil experiments. The gallium ratio is the gallium concentration (in atoms $\mathrm{cm}^{-2}$ ) on the type of collector foil listed divided by the gallium concentration at a corresponding point on a pure graphite foil which was mounted simultaneously during the sputtering run. A similar definition applies to the indium ratio. A correction for foil thickness needed to be made because the foil surfaces were not all the same distance from the incident $\mathrm{Ar}^{+}$beam spot. 


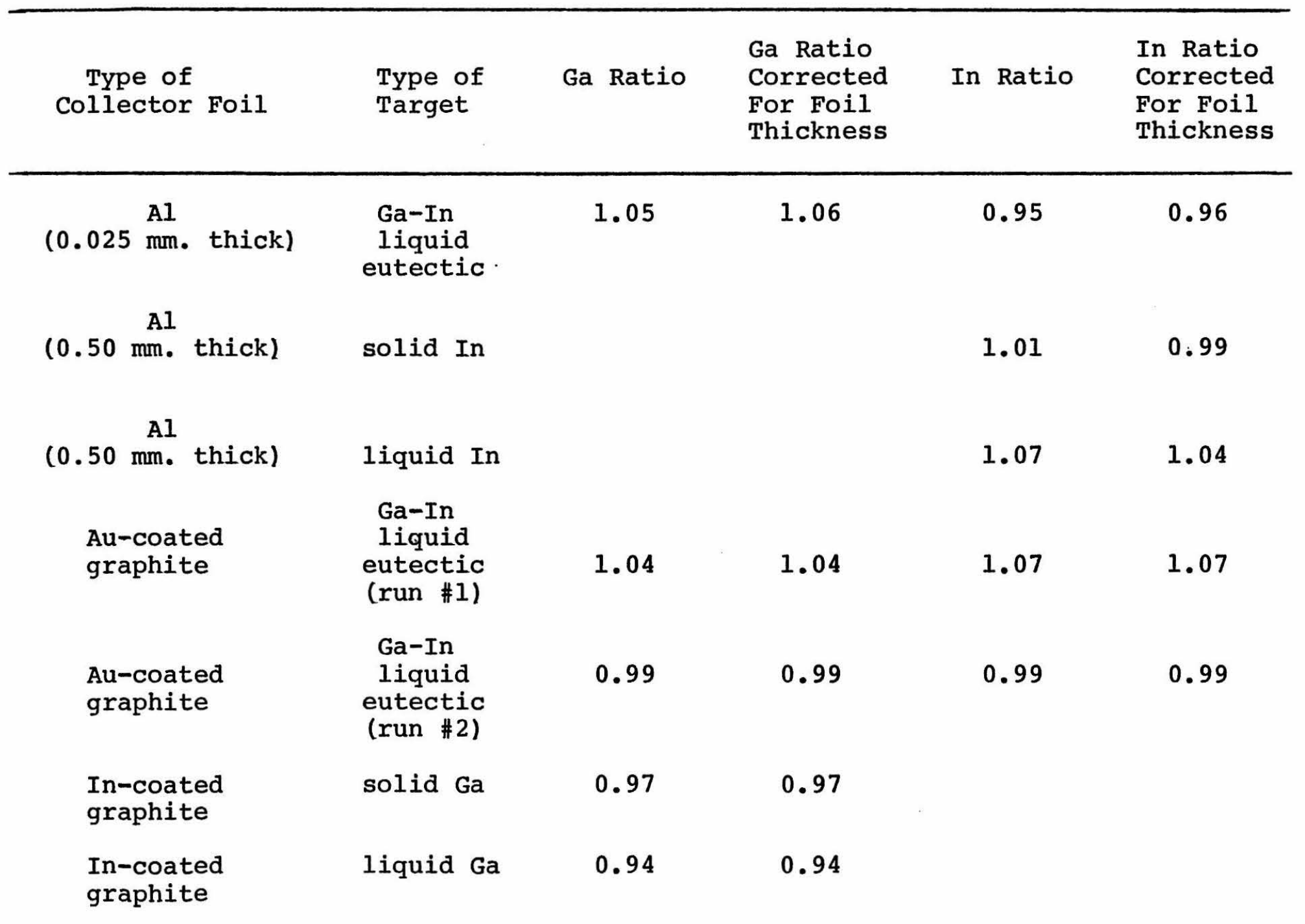




\section{Table 2}

Results of sputtering pure gallium and pure indium in both the solid and liquid phases. The sputtering yield $\mathrm{S}$ is the number of atoms ejected from the target divided by the number of incident $\mathrm{Ar}^{+}$ions. B is obtained by fitting the differential sputtering yield $d s / d \Omega$ to a function of the form $A \cos ^{B} \theta$ where $\theta$ is the angle with respect to the target normal. The uncertainty in $B$ is $\sigma_{B}$ which is obtained from the same linear regression used in determining $B$. 
TABLE 2

\begin{tabular}{|c|c|c|c|c|}
\hline Target & $\begin{array}{c}\text { Target } \\
\text { Temperature } \\
\left({ }^{\circ} \mathrm{C}\right)\end{array}$ & $\begin{array}{l}\text { Incident } \\
\mathrm{Ar}^{+} \text {Dose } \\
\text { (mCoulombs) }\end{array}$ & $\begin{array}{c}\text { Sputtering } \\
\text { Yield } \\
\text { S }\end{array}$ & $\begin{array}{c}\text { Angular } \\
\text { Distribution } \\
\text { Fit B }\end{array}$ \\
\hline Solid Ga & 11.0 & 5.00 & $3.8 \pm 0.2$ & $1.94 \pm 0.10$ \\
\hline Solid Ga & 12.5 & 10.0 & $3.9 \pm 0.2$ & $1.85 \pm 0.07$ \\
\hline Liquid $\mathrm{Ga}$ & 32.0 & 10.0 & $4.2 \pm 0.2$ & $1.96 \pm 0.07$ \\
\hline Iiquid $\mathrm{Ga}$ & 37.8 & $\begin{array}{l}\text { incorrect } \\
\text { beam-current } \\
\text { integration }\end{array}$ & & $1.92 \pm 0.08$ \\
\hline Solid In & 16.5 & 5.00 & $5.7 \pm 0.3$ & $1.94 \pm 0.05$ \\
\hline Liquid In & 164.0 & 5.00 & $5.4 \pm 0.3$ & $2.00 \pm 0.05$ \\
\hline
\end{tabular}


Table 3

Results of sputtering the liquid gallium-indium eutectic alloy. The partial sputtering yield is the number of atoms of a certain kind (either gallium or indium) which are ejected from the target divided by the number of incident $\mathrm{Ar}^{+}$ions. $B$ is obtained by fitting the partial differential sputtering yield to a function of the form $A \cos ^{B} \theta$. 
TABLE 3

\begin{tabular}{cccccc}
\hline $\begin{array}{c}\text { Temperature } \\
\text { of the Liquid } \\
\text { Ga-In Eutectic } \\
\left.\text { Target ( }{ }^{\circ} \mathrm{C}\right)\end{array}$ & $\begin{array}{c}\text { Incident } \\
\mathrm{Ar}^{+} \\
\text {Dose } \\
\text { (mCoulombs) }\end{array}$ & $\begin{array}{c}\text { Incident } \\
\mathrm{Ar}^{+} \\
\text {Energy } \\
(\mathrm{keV})\end{array}$ & $\begin{array}{c}\text { Type of } \\
\text { Sputtered } \\
\text { Atoms } \\
\text { (Ga or In) }\end{array}$ & $\begin{array}{c}\text { Partial } \\
\text { Sputtering } \\
\text { Yield }\end{array}$ & $\begin{array}{c}\text { Angular } \\
\text { Distribution } \\
\text { Fit B }\end{array}$ \\
\hline $9.6 *$ & 10.0 & 14.7 & Ga & $0.66 \pm 0.03$ & $3.6 \pm 0.2$ \\
31.8 & 10.0 & 14.7 & Ga & $3.7 \pm 0.2$ & $2.00 \pm 0.05$ \\
& & & In & $3.5 \pm 0.2$ & $1.99 \pm 0.05$ \\
27.0 & 3.33 & 24.7 & Ga & $0.95 \pm 0.05$ & $2.0 \pm 0.2$ \\
& & & In & $2.5 \pm 0.1$ & $1.98 \pm 0.09$
\end{tabular}

*The melting point of the eutectic alloy was $15.7^{\circ} \mathrm{C}$. However, the liquid alloy remained in superfusion until cooled to $0^{\circ} \mathrm{C}$. 


\section{Table 4}

Results of sputtering the solid gallium-indium eutectic alloy. The total accumulated $\mathrm{Ar}^{+}$dose before the beginning and by the end of each run is included. The incident $\mathrm{Ar}^{+}$ dose for each run may be obtained by taking the difference of the total accumulated doses. The partial sputtering yield is the number of atoms of a certain kind which are ejected from the target divided by the number of incident $\mathrm{Ar}^{+}$ions. B is obtained by fitting the partial differential sputtering yield to a function of the form $A \cos ^{B} \theta$. The data above the dashed line pertain to the target which was frozen gradually. The data below the dashed line are taken from the quickly frozen target. 
TABLE 4

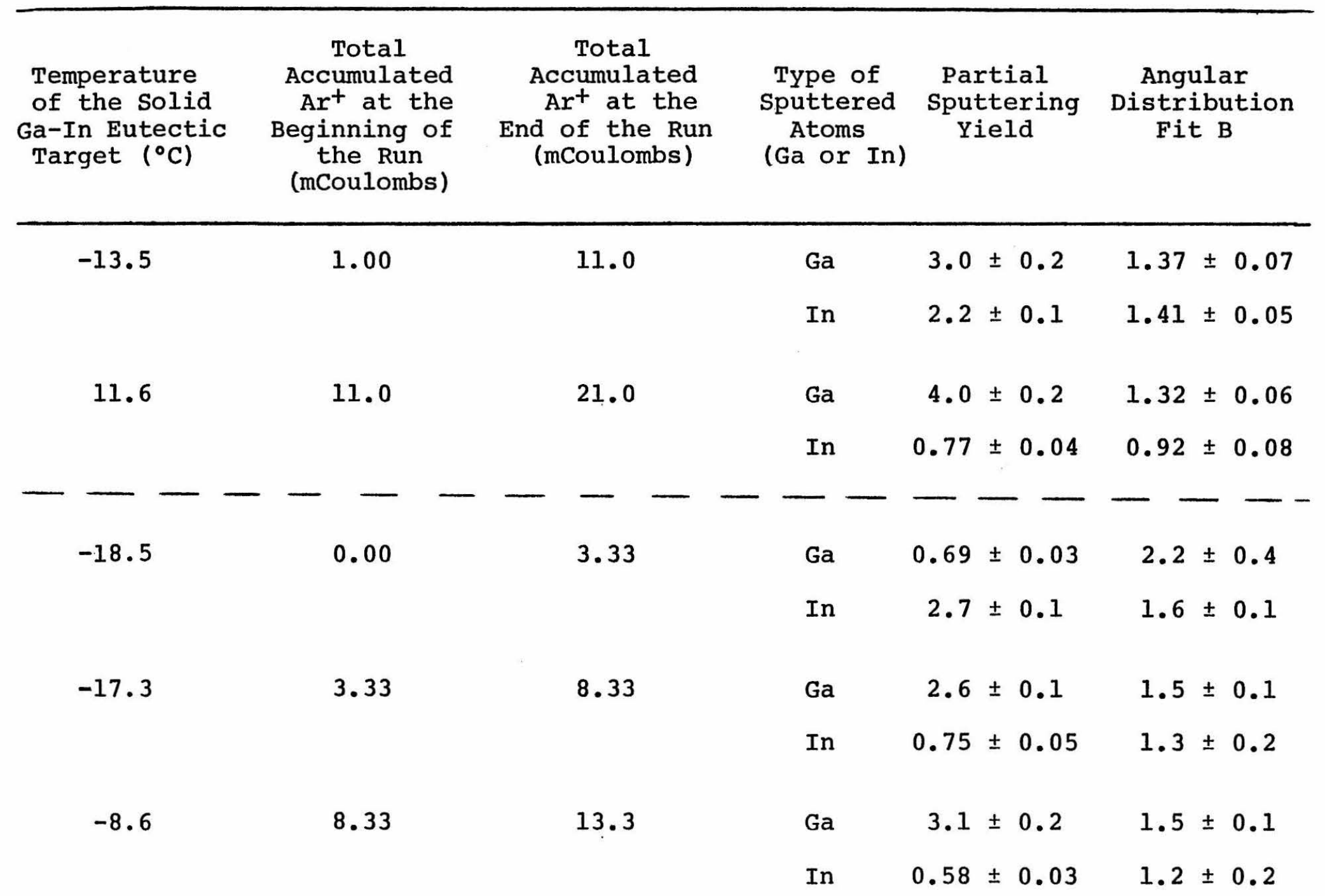


Figure 1

An illustration of the apparatus which was constructed in order to sputter liquid targets. The drawing is scaled down by approximately a factor of 15 . 


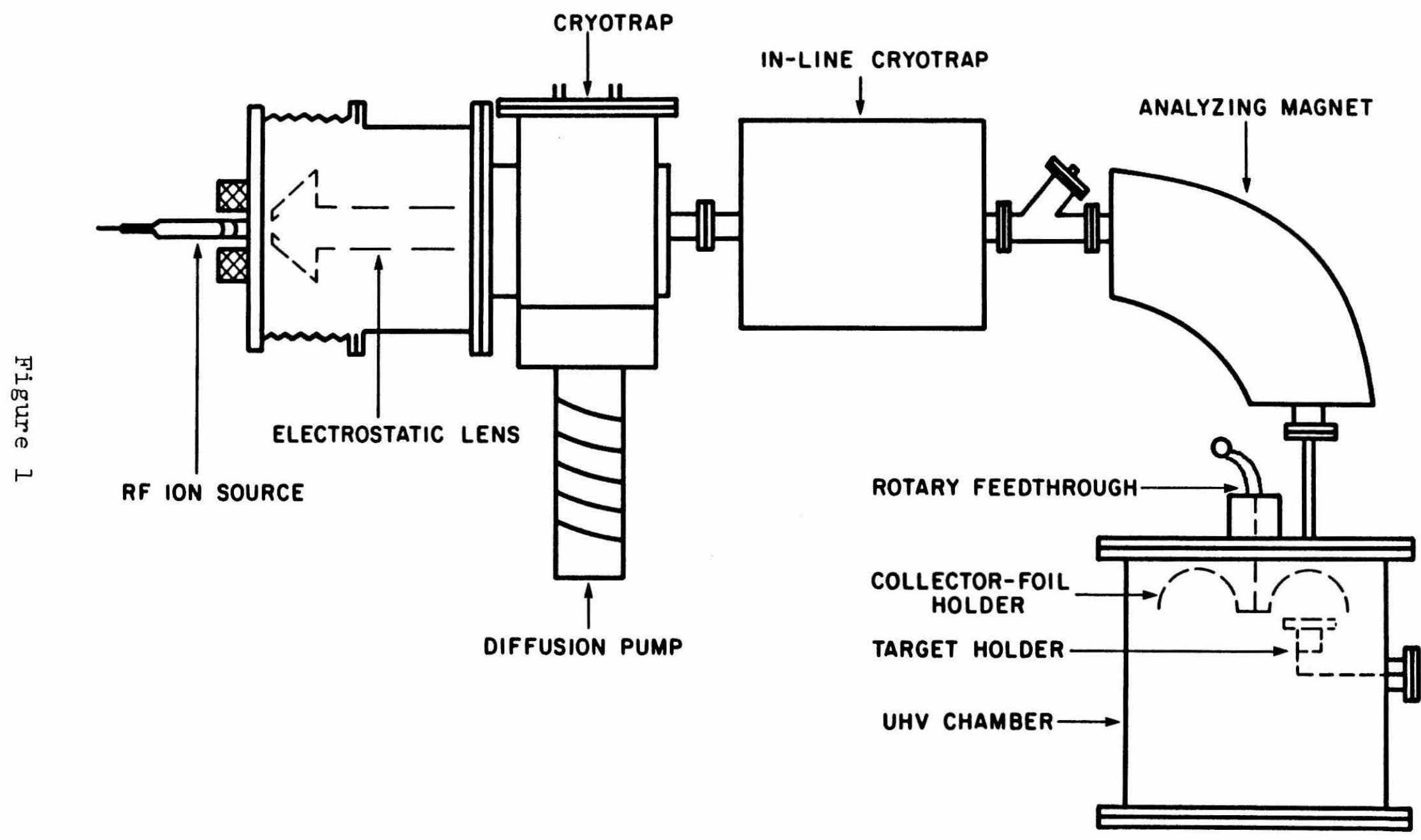


Figure 2

Photograph of the apparatus which was constructed in order to sputter liquid targets. 


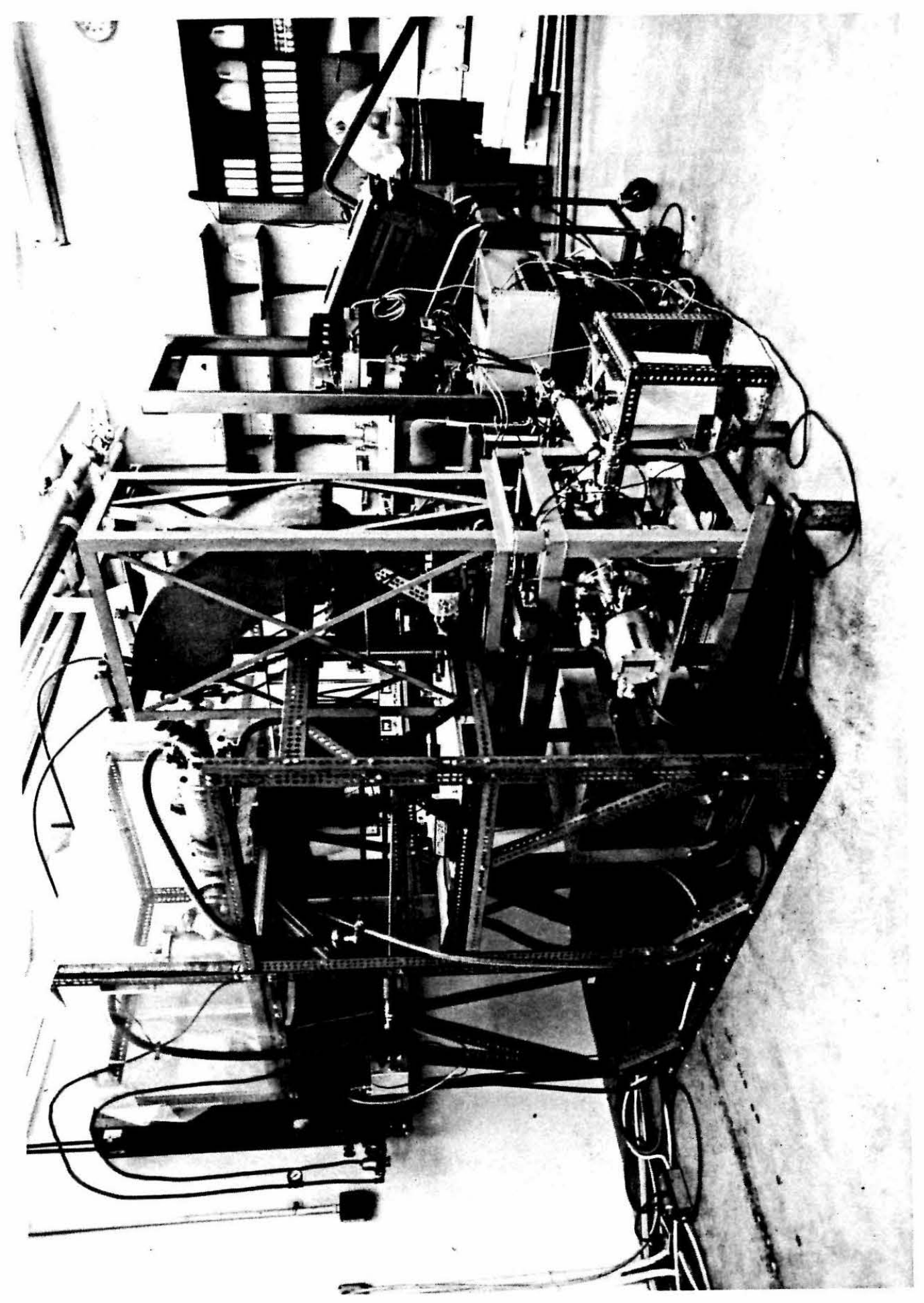




$$
-69-
$$

Figure 3

Photograph of the radio-frequency ion source which produced the beams used for sputtering and for ion scattering spectroscopy. 


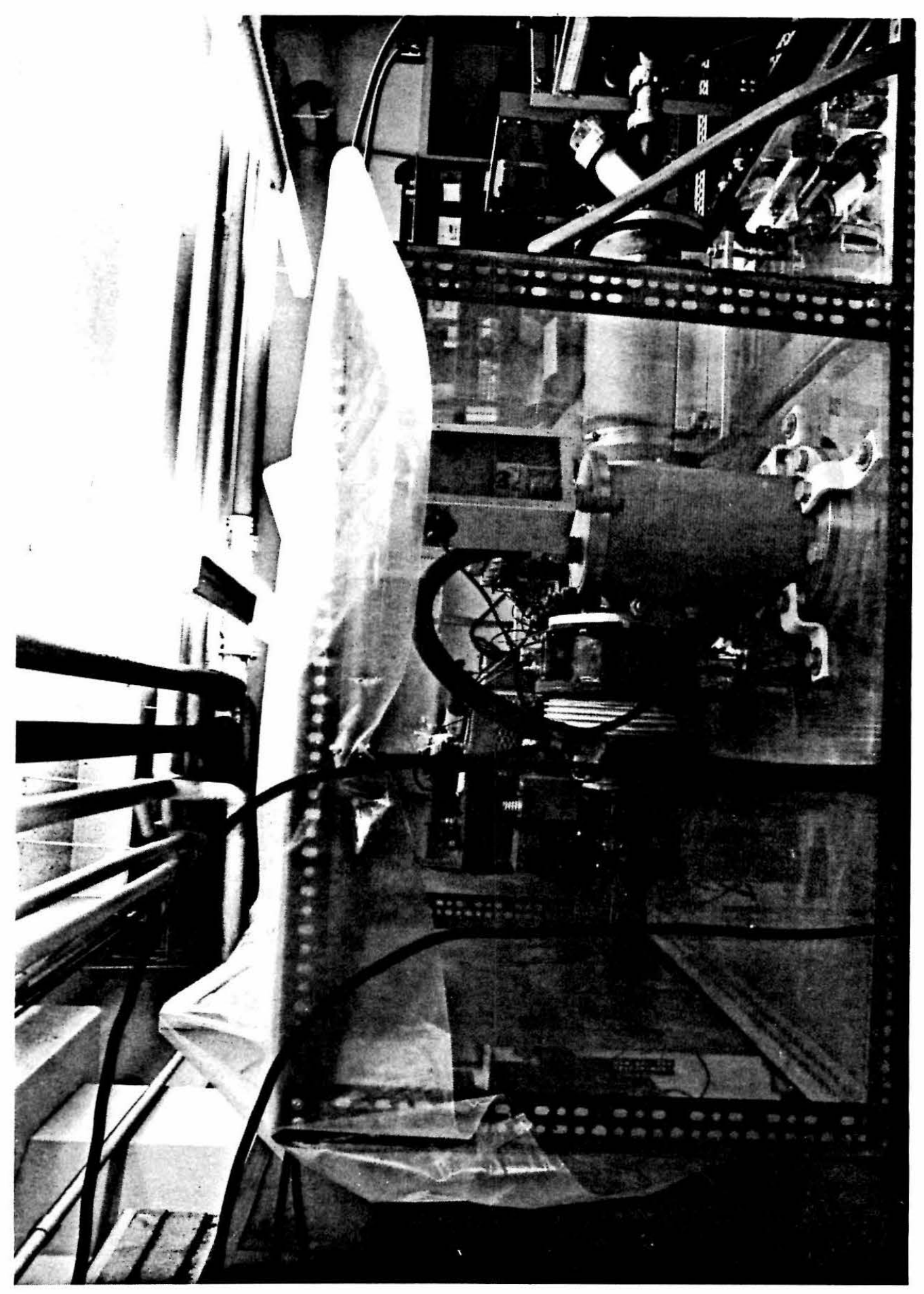

Tigure 3 
Figure 4

Schematic drawing of the target and collector-foil holders used during sputtering. The tungsten heating filament for the target holder is completely encased in machineable glass-ceramic. The incident beam normally used was $15 \mathrm{keV}$ $\mathrm{Ar}^{+}$. 


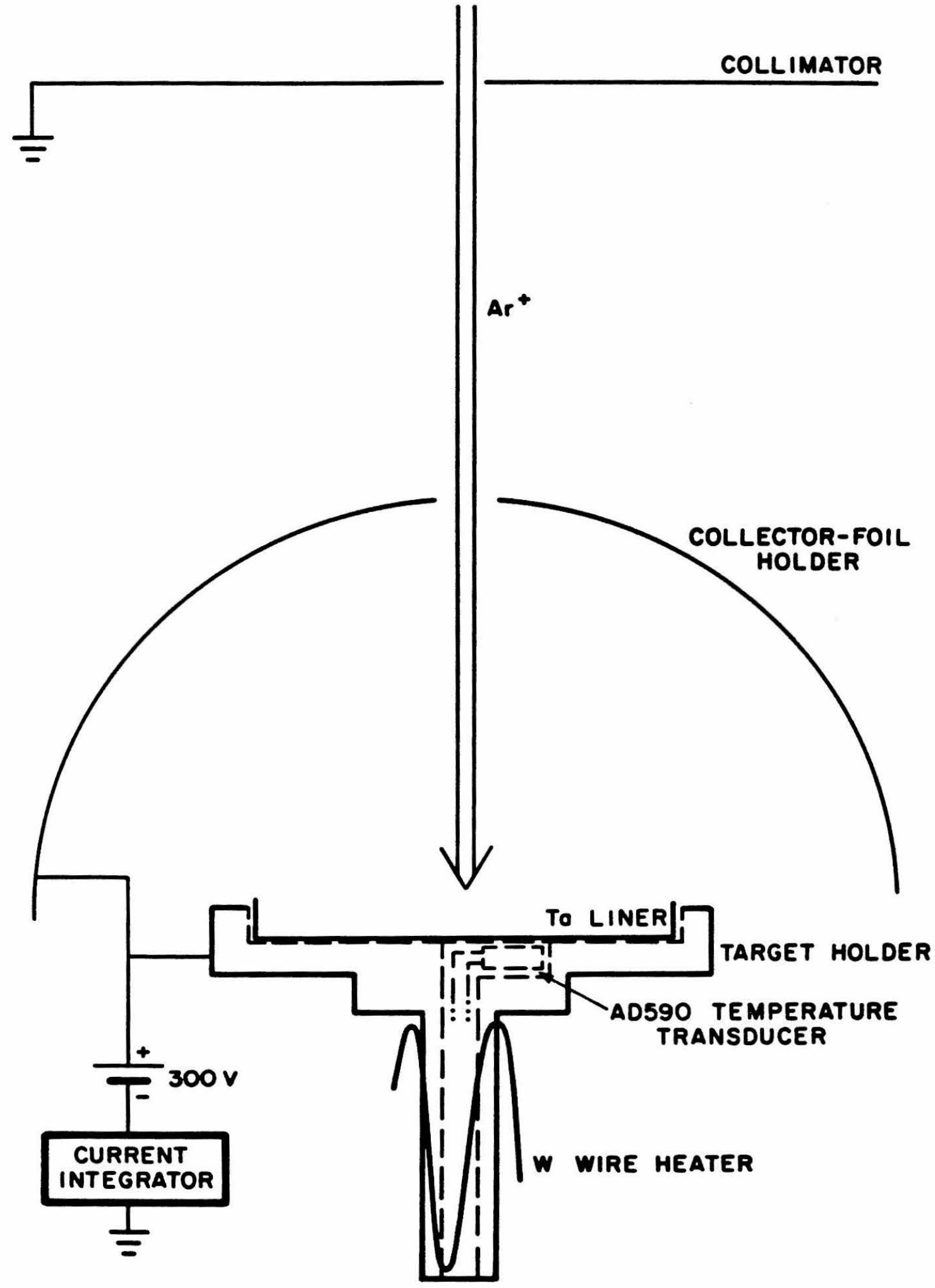


Figure 5

Diagram of the temperature-controller circuit. The heater displayed here is the tungsten filament shown in Figure 4 . 


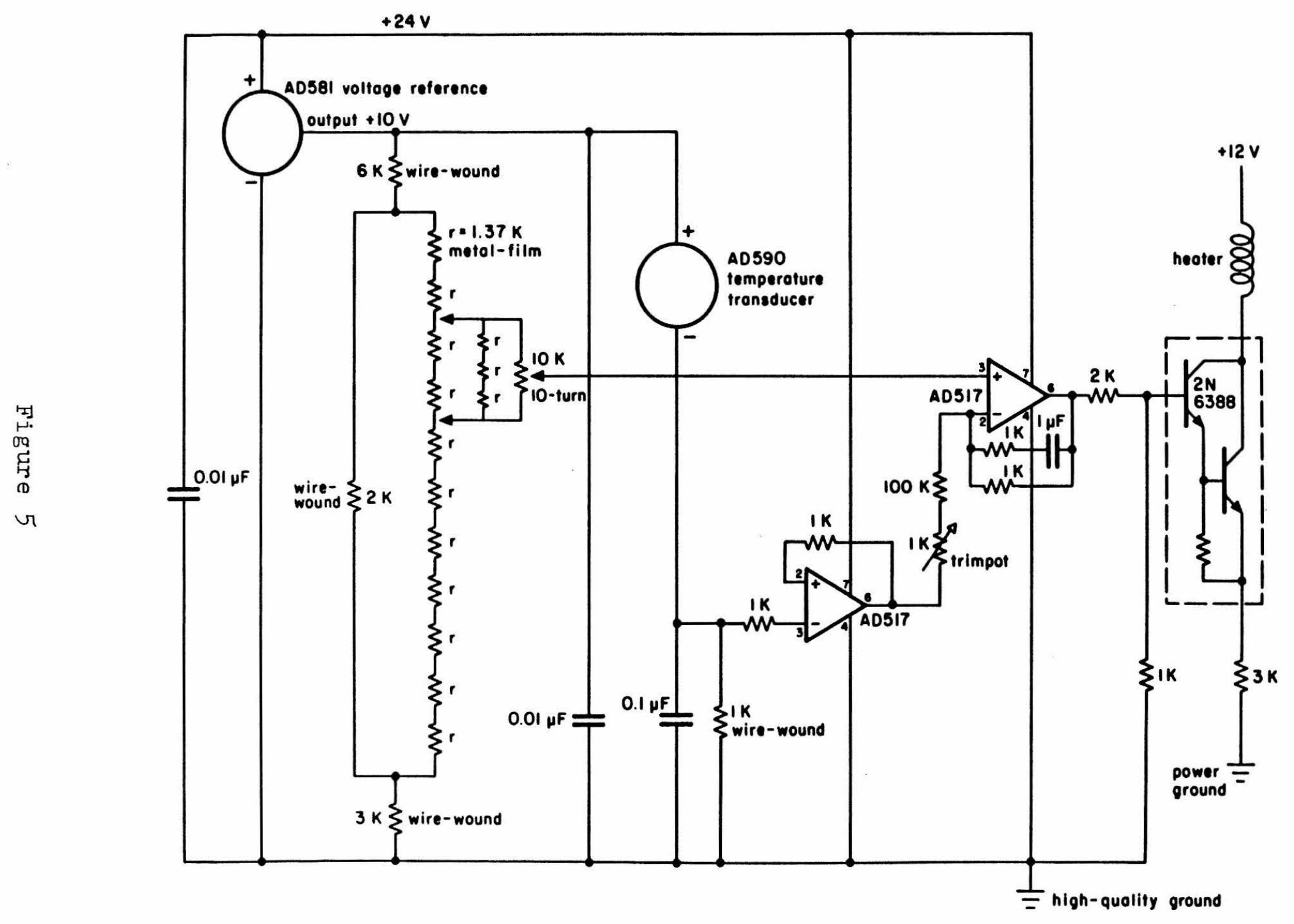




\section{Figure 6}

Schematic drawing of the Rutherford backscattering apparatus. The target holder rotates about an axis which is perpendicular to the plane of the diagram. The incident beam normally used was $5.00 \mathrm{MeV}{ }^{19} \mathrm{~F}^{2+}$. The backscattered yields were measured using a silicon surface-barrier detector. 


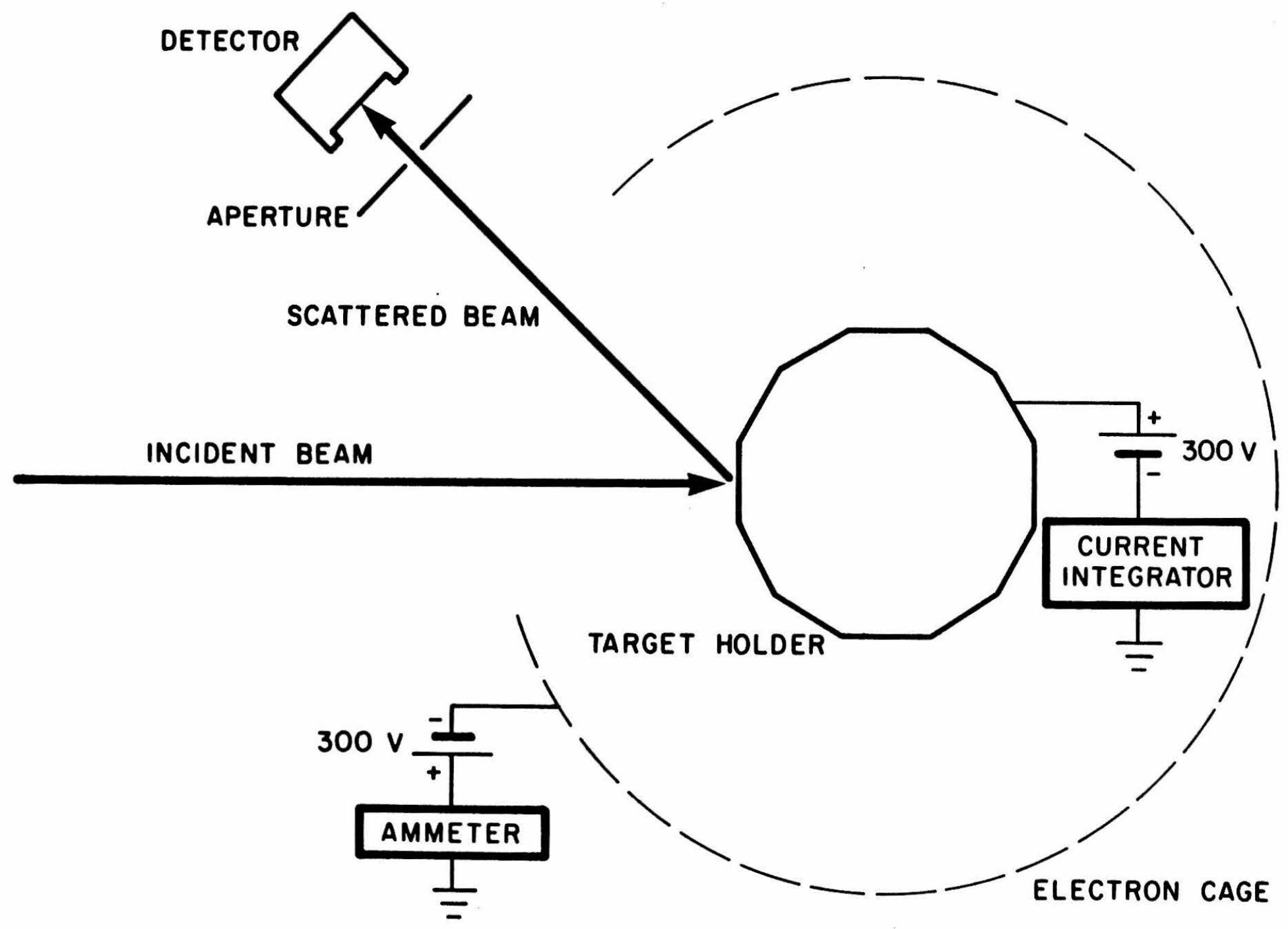


$-77-$

Figure 7

The normalized differential sputtering yield versus $\theta$ for the low-dose solid gallium run of Table 2. The uncertainty in the yields is 58 which includes the systematic errors discussed in Chapter III. The uncertainty in $\theta / \pi$ is 0.001 . The continuous line is $\cos ^{1 \cdot 94} \theta$. 


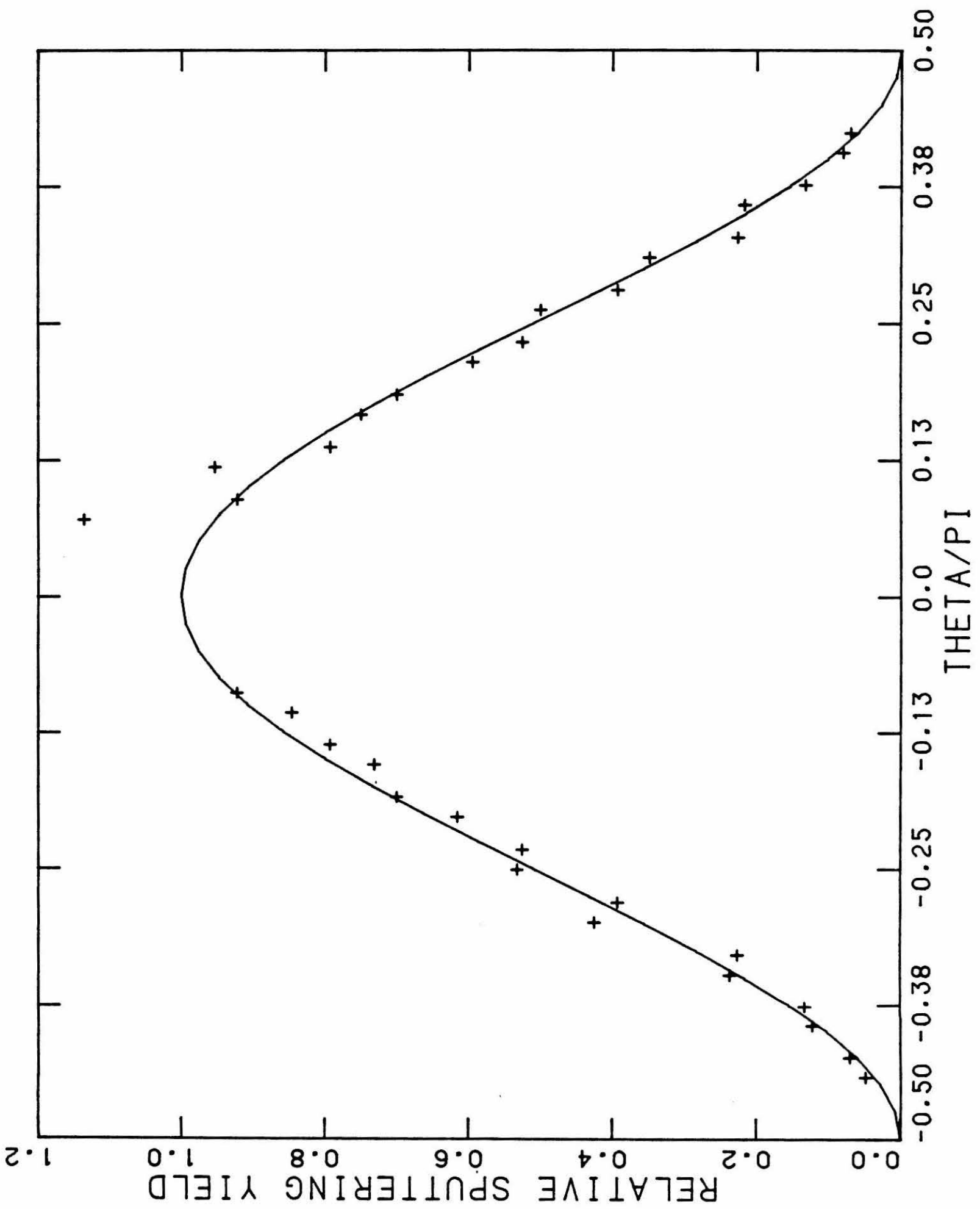




\section{Figure 8}

The normalized differential sputtering yield versus $\theta$ for the high-dose solid gallium run of Table 2. The uncertainties are as quoted in the previous figure caption. The continuous line is $\cos ^{1.85} \theta$. 


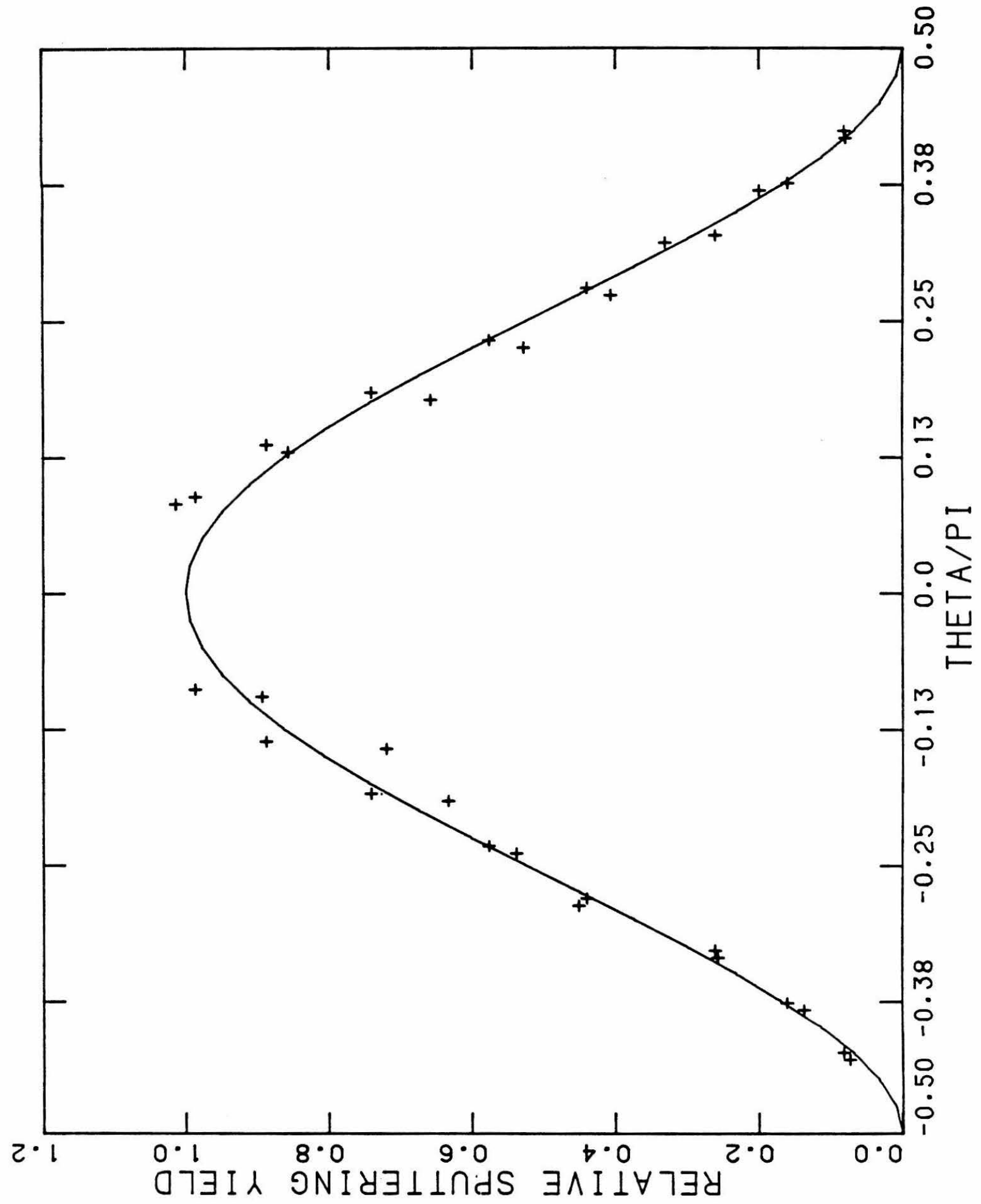

Figure 8 
Figure 9

The normalized differential sputtering yield versus $\theta$ for the first liquid gallium run listed in Table 2. The continuous line is $\cos ^{1.96} \theta$. 


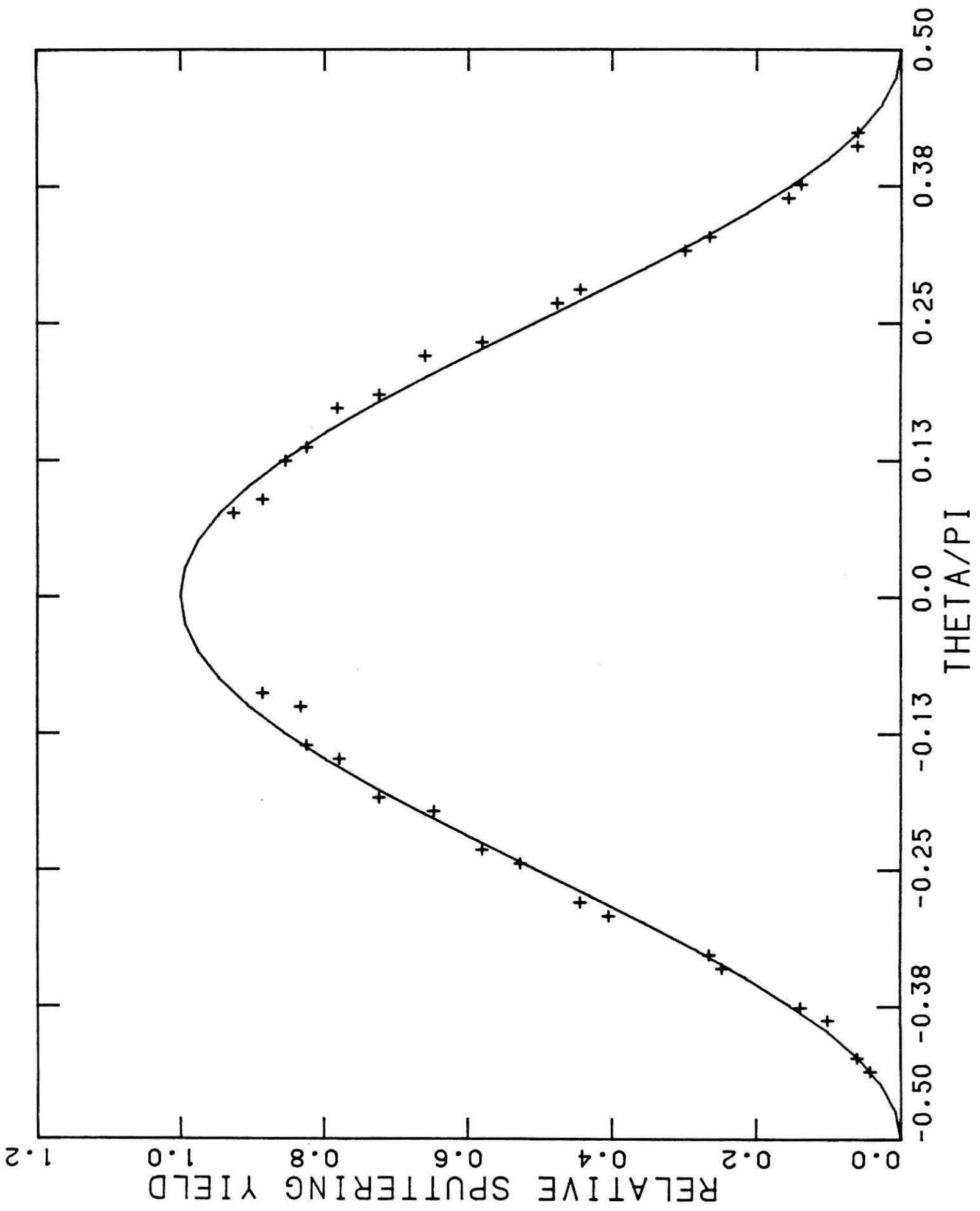

Figure 9 
$-83-$

Figure 10

The normalized differential sputtering yield versus $\theta$ for the second liquid gallium run listed in Table 2. Although no sputtering yield could be obtained for this run due to poor $\mathrm{Ar}^{+}$beam current integration, the angular distribution is independent of beam current integration during sputtering. The continuous 1 in is $\cos ^{1.22} \theta$. 


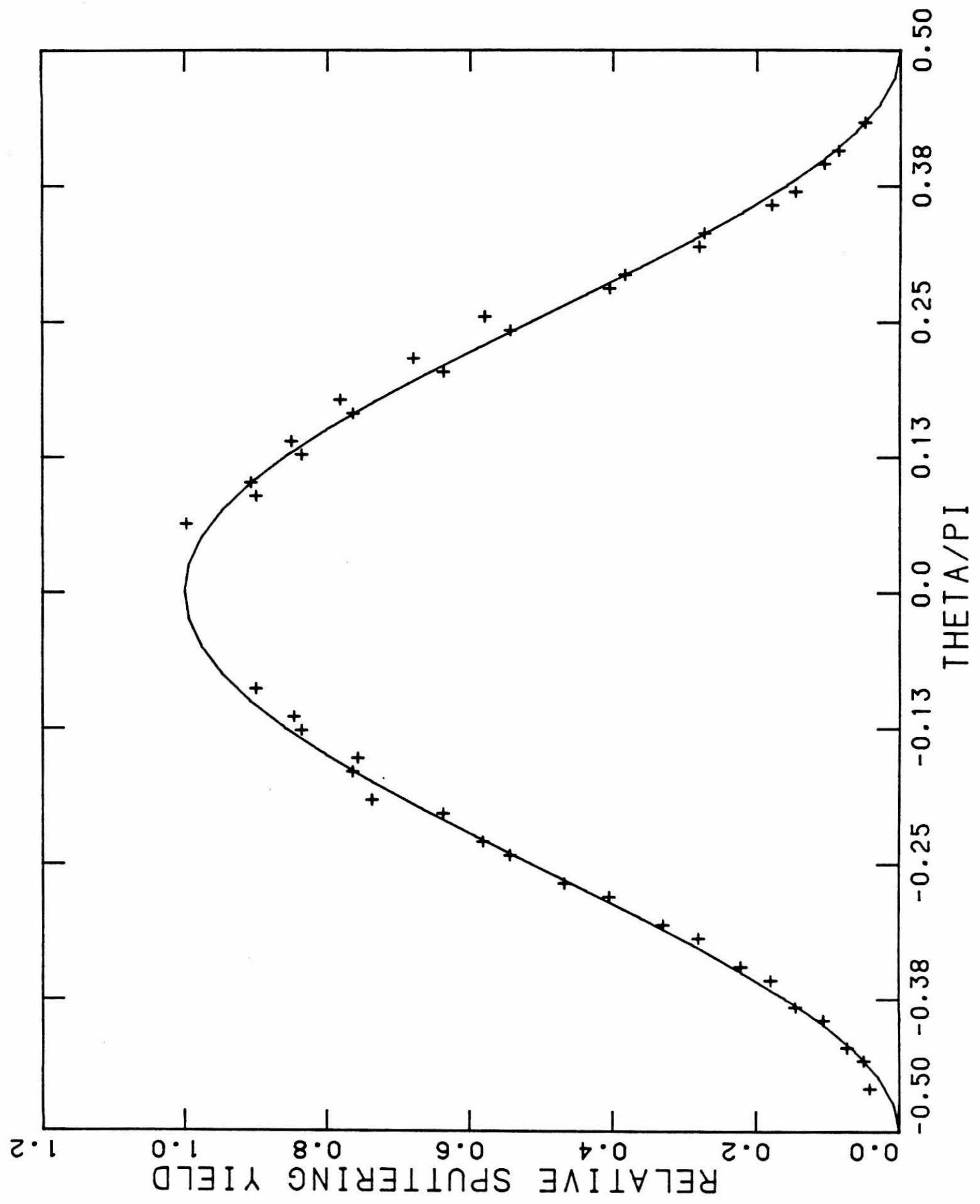




$$
-85-
$$

\section{Figure 11}

The normalized differential sputtering yield versus $\theta$ for the solid indium run in Table 2. The estimated uncertainty in yields is 5\%, and the uncertainty in $\theta / \pi$ is 0.001 . The continuous line is $\cos ^{1 \cdot 94} \theta$. 


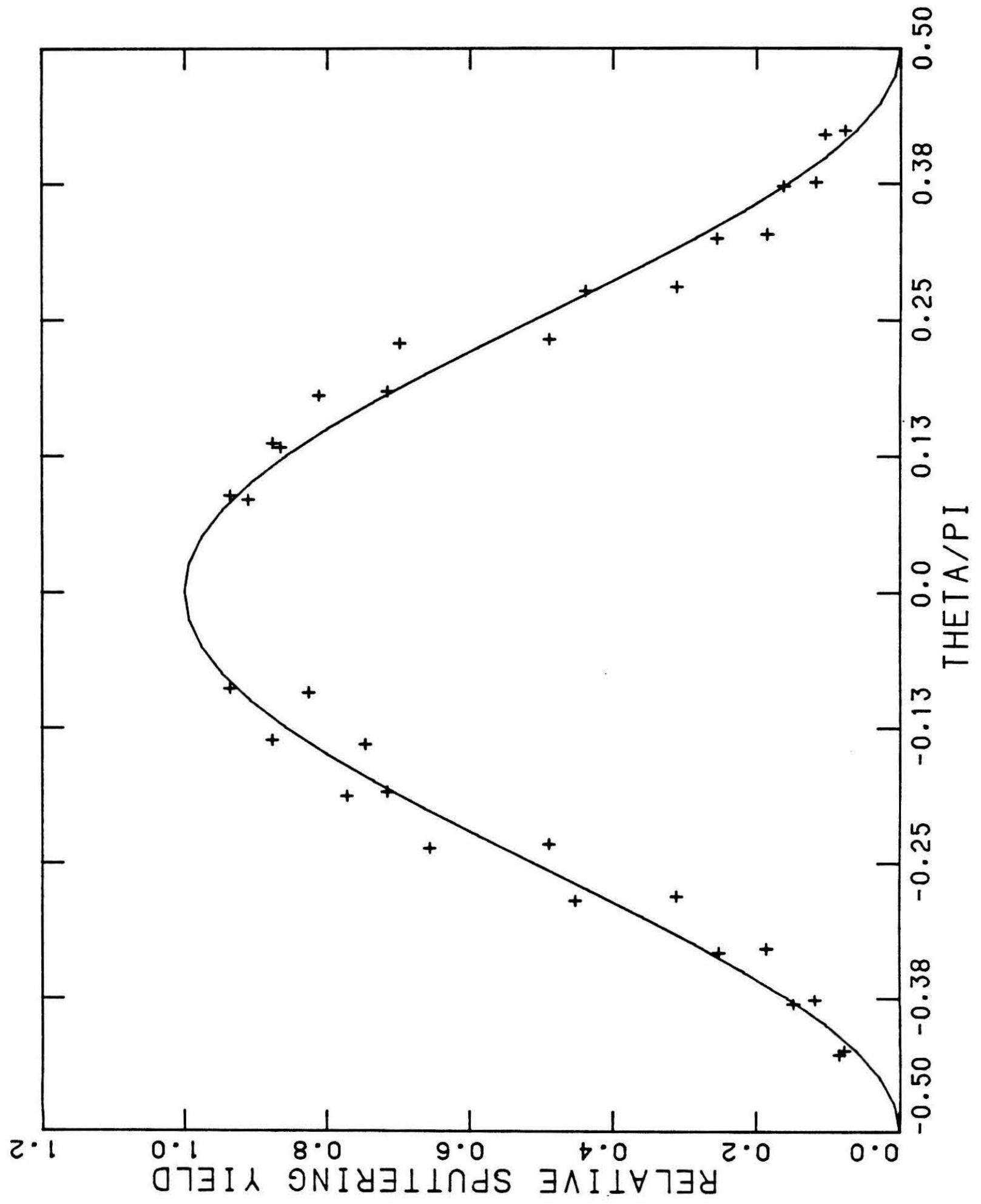

Figure 11 


$$
-87-
$$

\section{Figure 12}

The normalized differential sputtering yield versus $\theta$ for the liquid indium run in Table 2. The uncertainties are the same as in the previous figure caption. The continuous line is $\cos ^{2} \cdot \theta \cdot \theta$. 


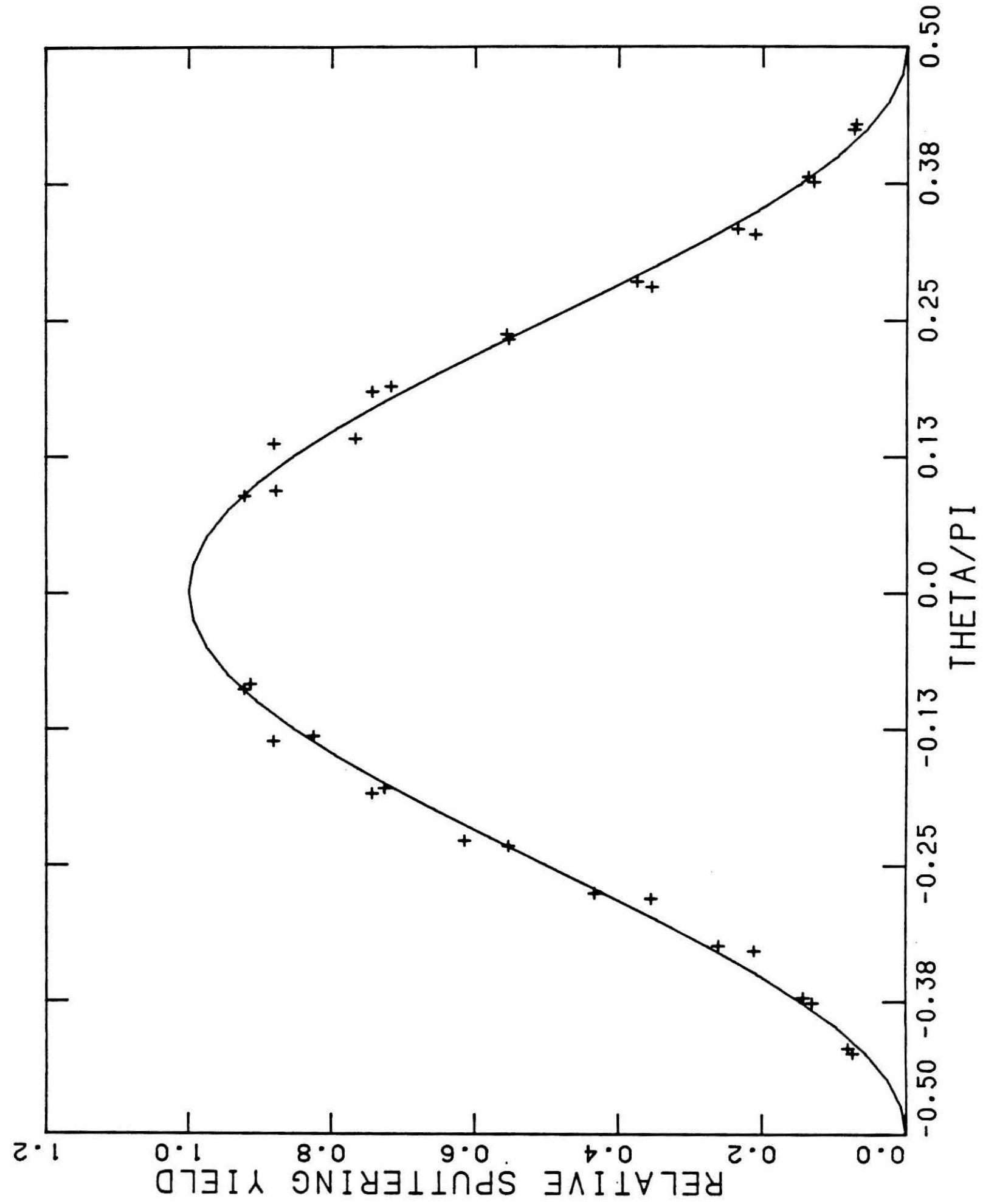




$$
-89-
$$

\section{Figure 13}

Rutherford backscattering spectrum of a graphite foil which collected sputtered atoms from a liquid gallium-indium eutectic target. The Rutherford cross section for indium is 2.5 times as large as the cross section for gallium. The incident ${ }^{19} \mathrm{~F}^{2+}$ energy was $5.00 \mathrm{MeV}$. 


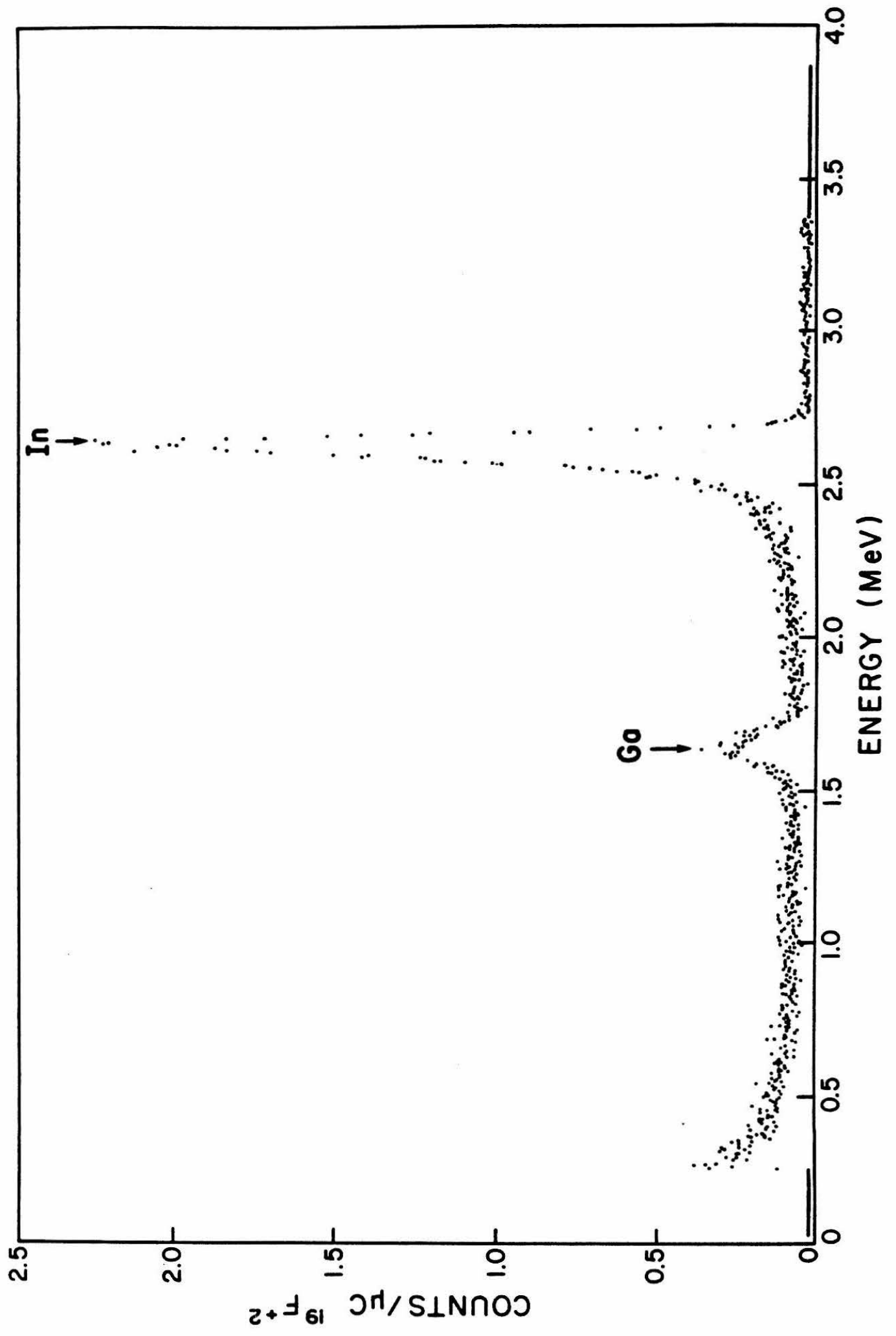

Figure 13 


\section{Figure 14}

The normalized differential sputtering yield versus $\theta$ for gallium sputtered from a liquid gallium-indium eutectic target by $15 \mathrm{keV} \mathrm{Ar}^{+}$. The target temperature was $31.8^{\circ} \mathrm{C}$ (see Table 3). The continuous line is $\cos ^{3} \cdot \theta$. Compare the angular distribution to the indium distribution taken from the same target in Figure 15. 


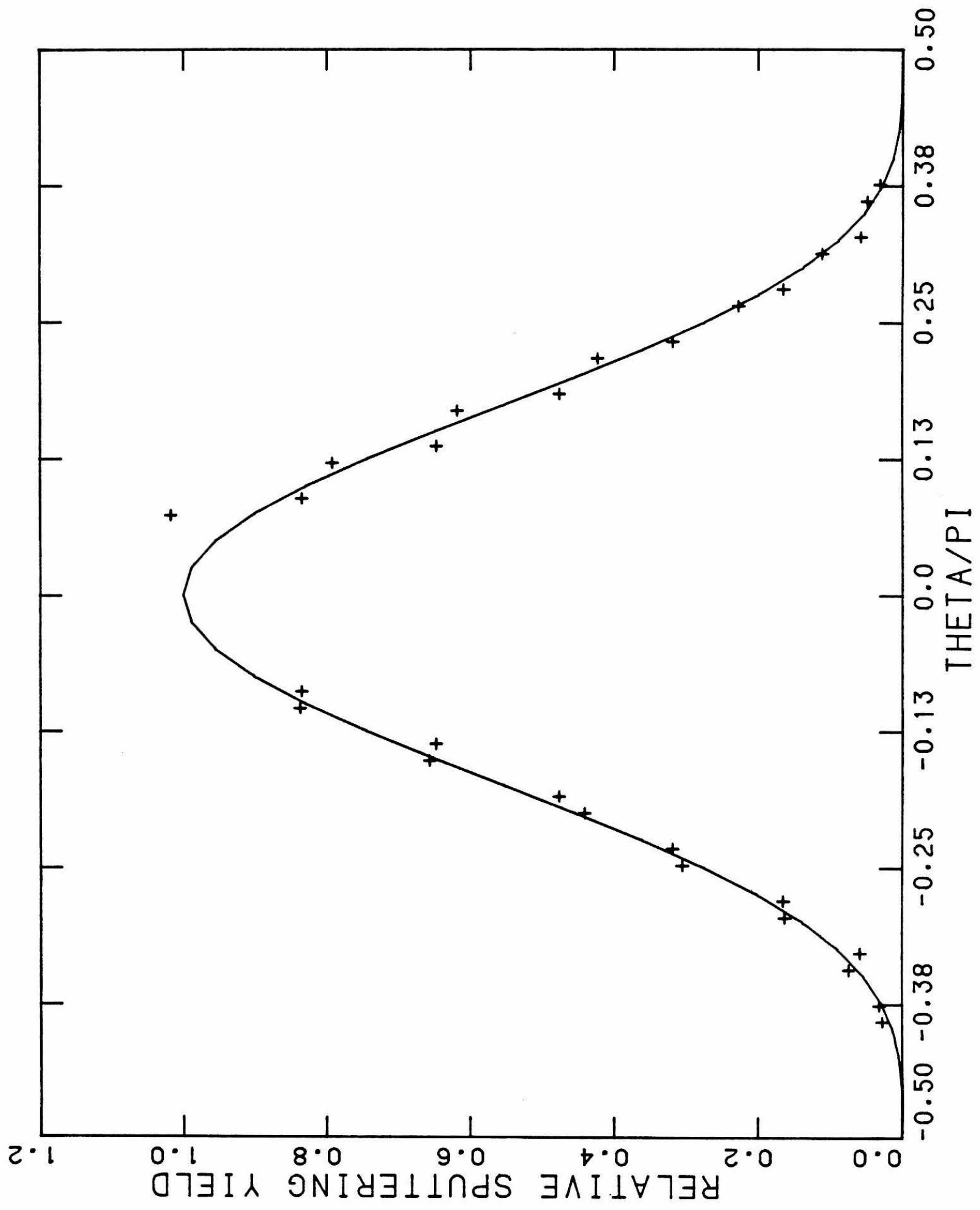

Figure 14 
Figure 15

The normalized differential sputtering yield versus $\theta$ for indium sputtered from the same liquid eutectic target as in Figure 14. The incident sputtering beam was $15 \mathrm{keV} \mathrm{Ar}^{+}$. The continuous line is $\cos ^{1.99} \theta$. 
$-94-$

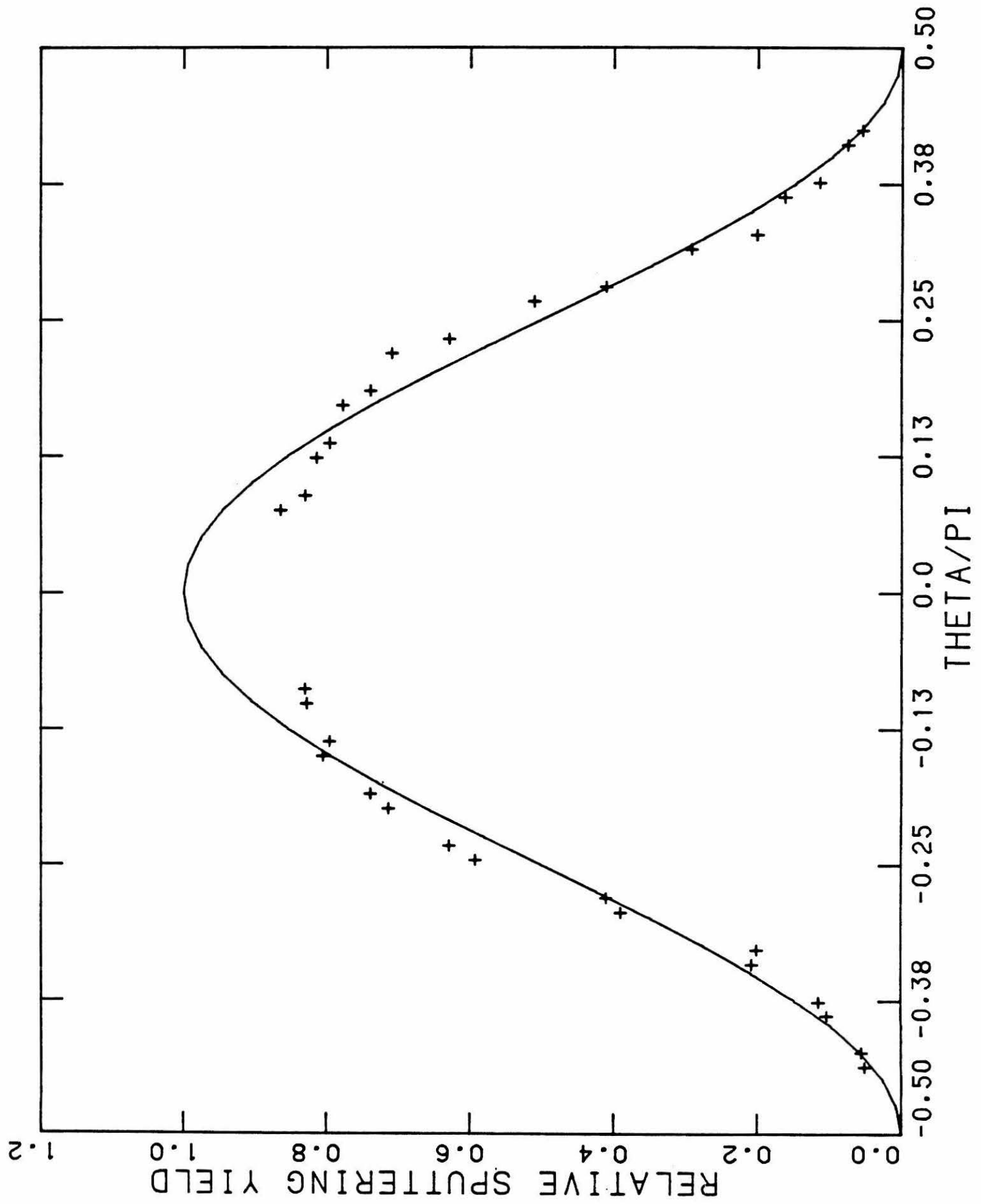

Figure I5 


\section{$-95-$}

Figure 16

The normalized differential sputtering yield versus $\theta$ for gallium sputtered from a liquid gallium-indium eutectic target by $25 \mathrm{keV} \mathrm{\textrm {Ar } ^ { + }}$ (see Table 3). The continuous line is $\cos ^{2} \cdot a \theta$. 
-96-

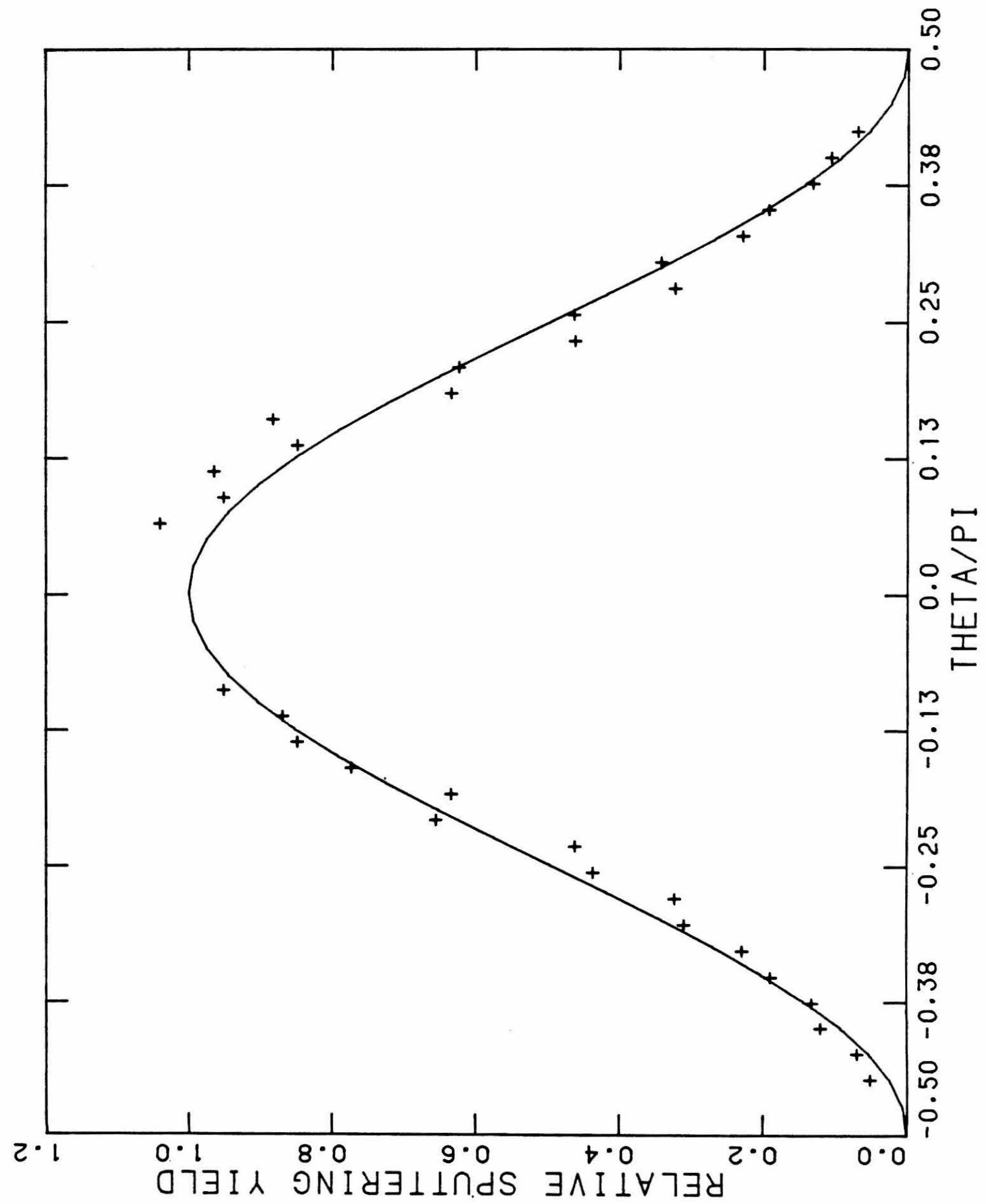

Figure 16 


\section{$-97-$}

\section{Figure 17}

The normalized differential sputtering yield versus $\theta$ for indium sputtered from the same liquid eutectic target and by the same $25 \mathrm{keV} \mathrm{Ar}^{+}$beam as in the previous figure. The continuous line is $\cos ^{1 \cdot 98} \theta$. 


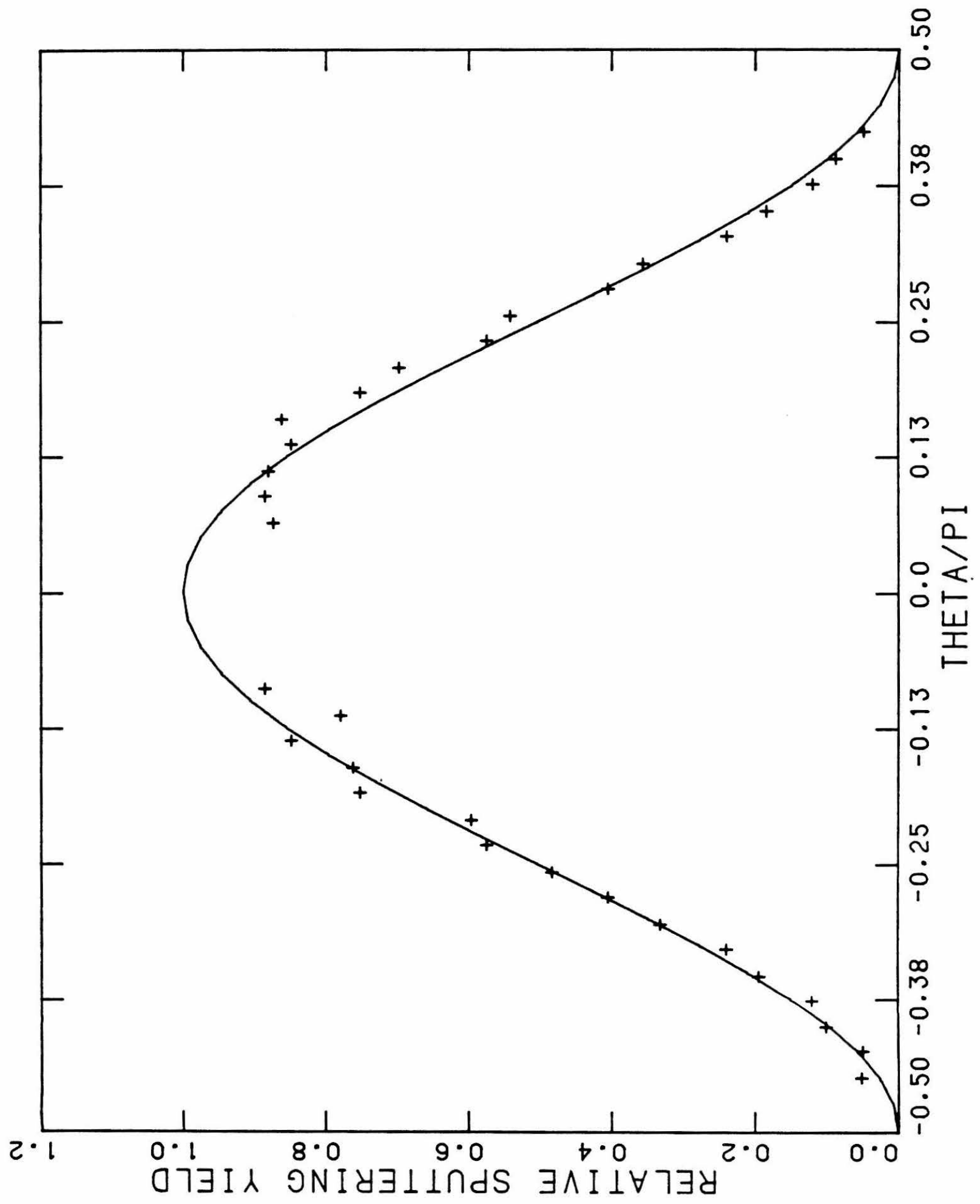

Figure 17 


\section{Figure 18}

Rutherford backscattering spectrum of a graphite foil which collected sputtered atoms from the first run on the quickly frozen eutectic target. The total accumulated $\mathrm{Ar}^{+}$ at the end of the run was 3.33 mCoulombs (see Table 4). The energy of the incident ${ }^{19} \mathrm{~F}^{2+}$ beam was $5.00 \mathrm{MeV}$. 


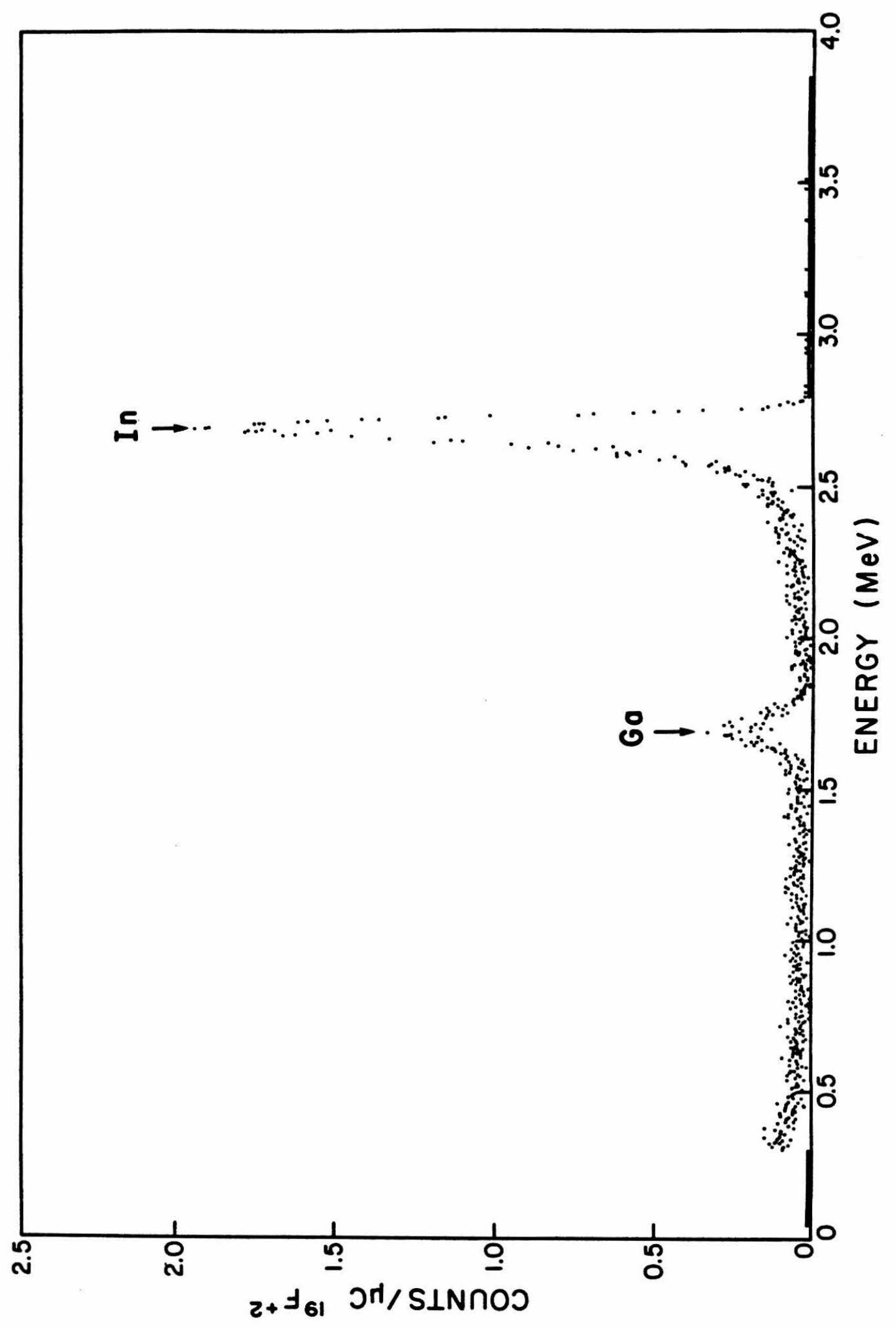


Figure 19

Rutherford backscattering spectrum of a graphite foil which collected sputtered atoms from the second run on the quickly frozen eutectic target. The total accumulated Ar+ at the end of the run was 8.33 mCoulombs (see Table 4). 


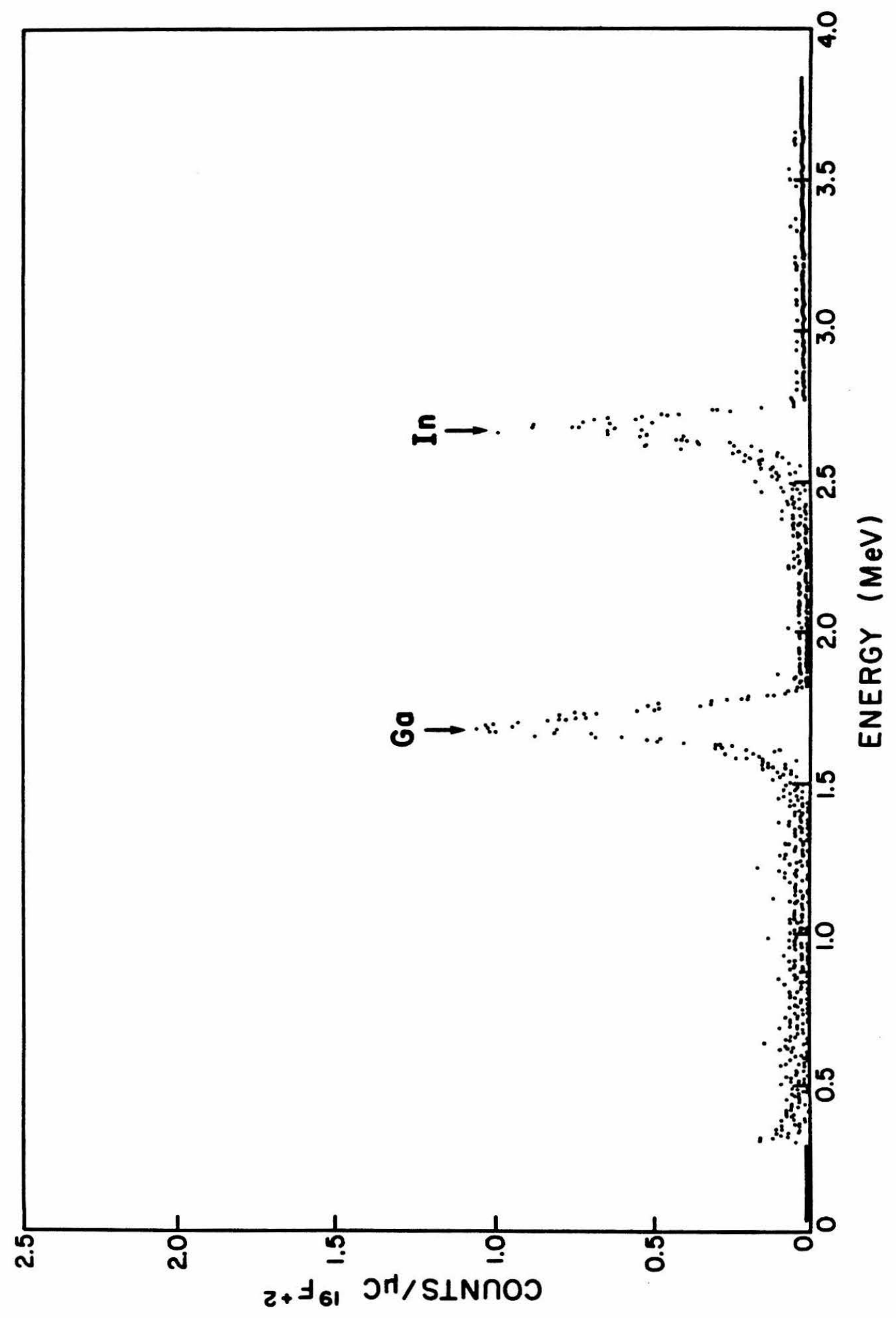

Figure 19 
Figure 20

Rutherford backscattering spectrum of a graphite foil which collected sputtered atoms from the third run on the quickly frozen eutectic target. The total accumulated $\mathrm{Ar}^{+}$ at the end of the run was 13.3 mCoulombs (see Table 4). 


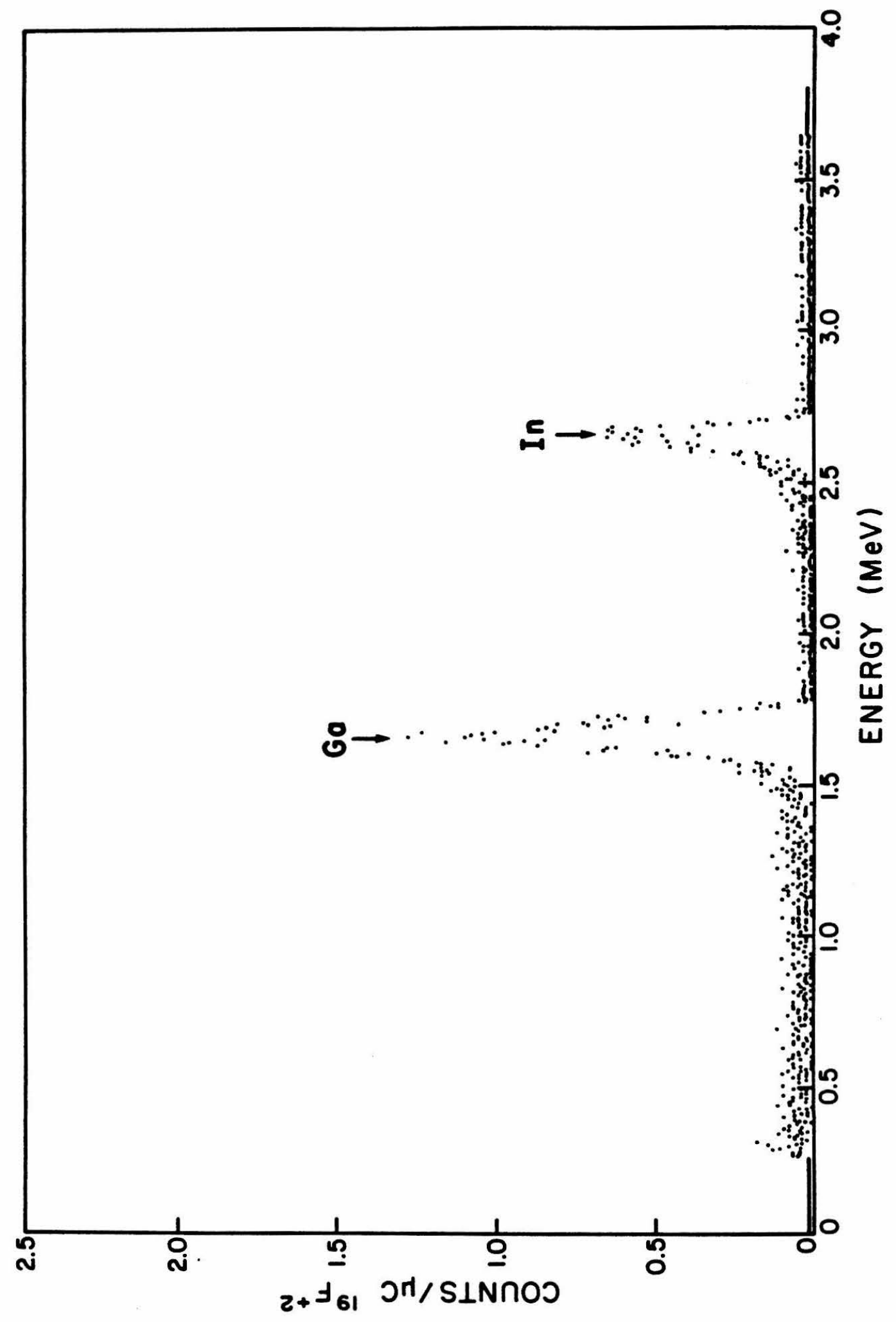

Figure 20 
Figure 21

The composition of the material sputtered from the solid gallium-indium eutectic versus incident $\mathrm{Ar}^{+}$dose. The shaded blocks represent sputtering runs of the quickly frozen target while the unshaded blocks represent the target which was frozen gradually. 


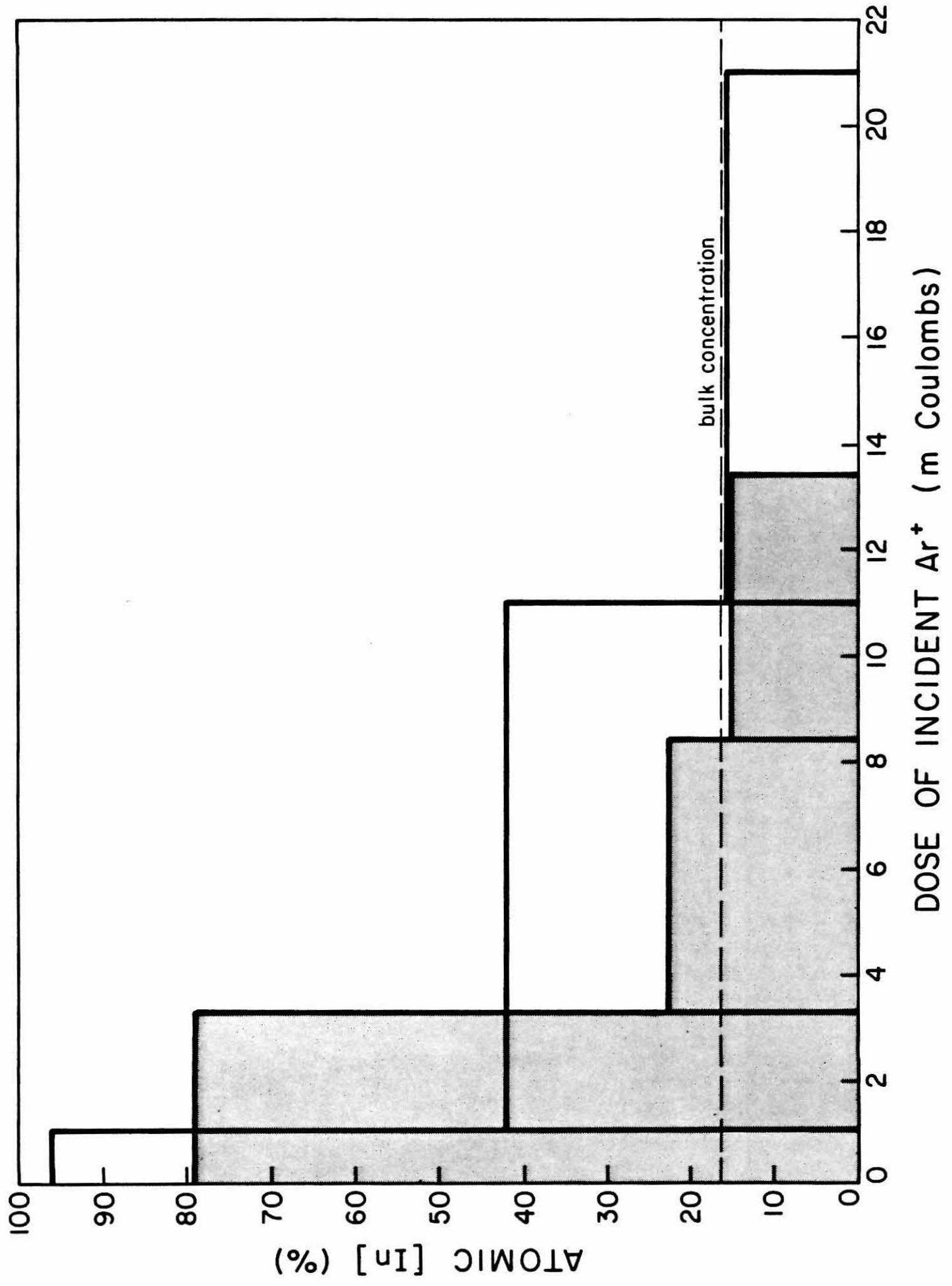

Figure 21 


\section{Figure 22}

The normalized differential sputtering yield versus $\theta$ for indium sputtered from the slowly frozen eutectic target during the first run listed in Table 4. The total accumulated $\mathrm{Ar}^{+}$at the end of the run was 11.0 mCoulombs. The continuous line is $\cos ^{1.41} \theta$. 


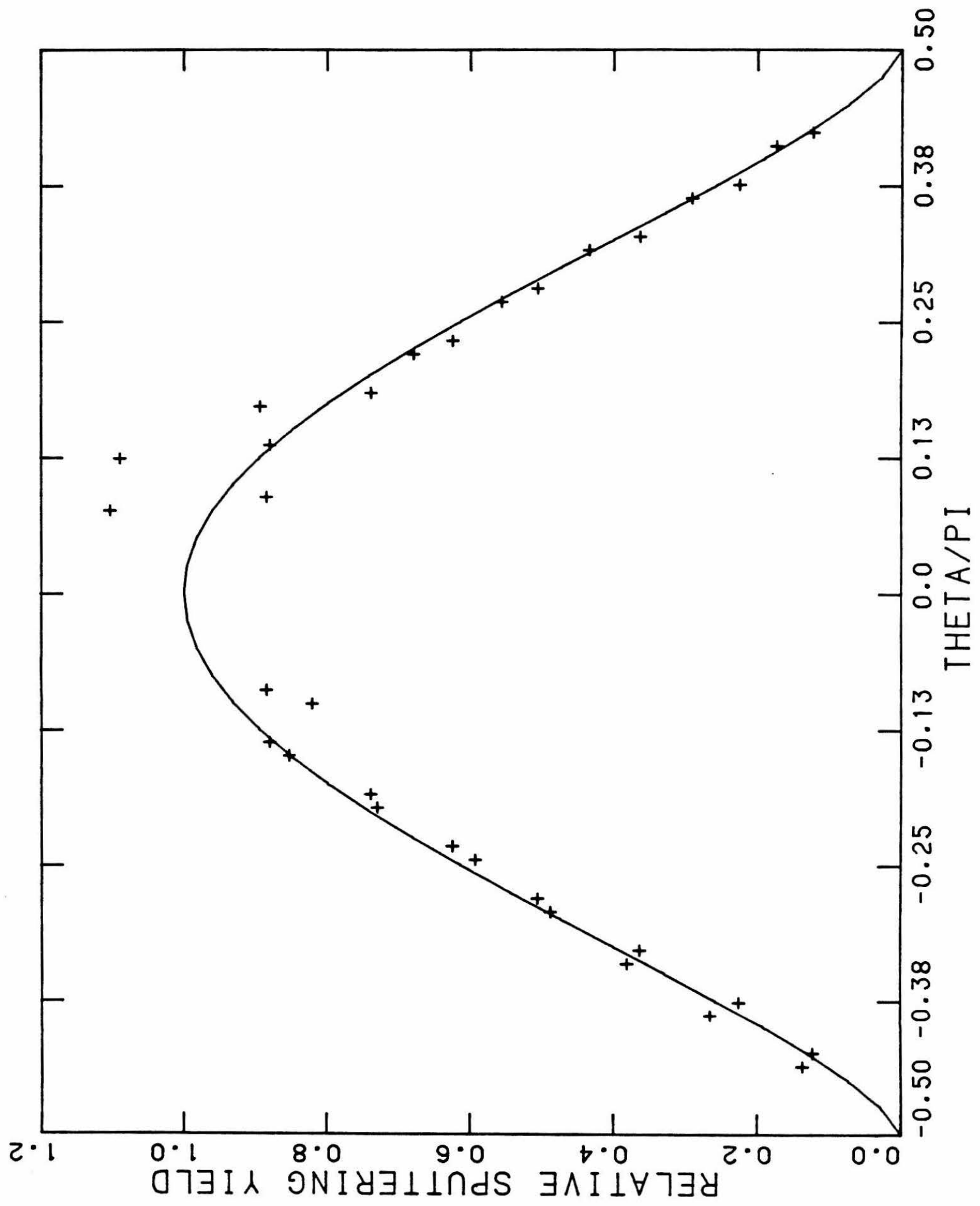

Figure 22 
Figure 23

The normalized differential sputtering yield versus $\theta$ for indium sputtered from the slowly frozen eutectic target during the second run listed in Table 4. The total accumulated $\mathrm{Ar}^{+}$at the end of the run was 21.0 mCoulombs. The continuous line is $\cos ^{0.92} \theta$. 


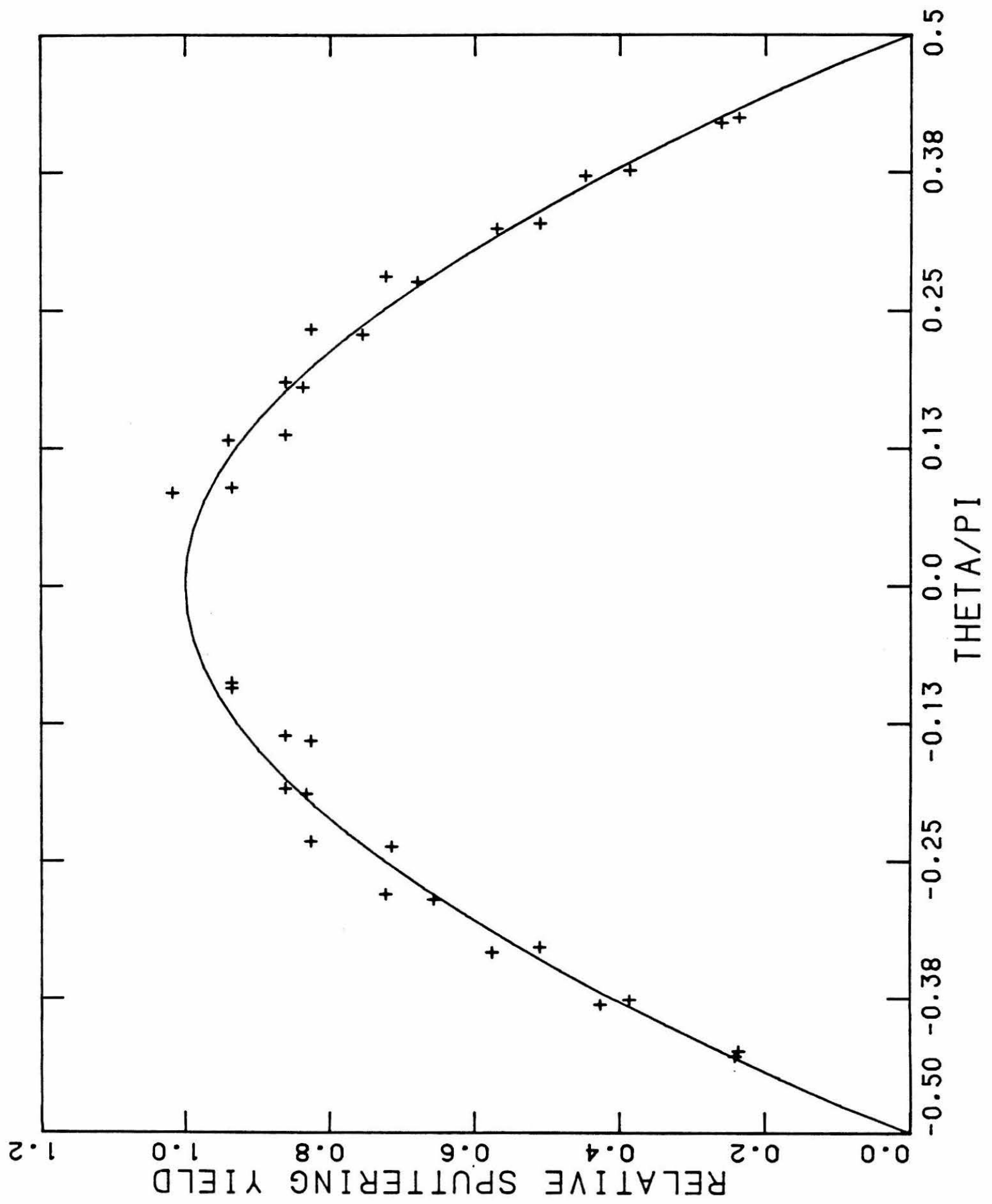

Figure 23 
Figure 24

The normalized differential sputtering yield versus $\theta$ for indium sputtered during the first run of the quickly frozen eutectic target. The total accumulated $\mathrm{Ar}^{+}$at the end of the run was 3.33 mCoulombs. The continuous line is $\cos ^{1.6} \theta$. 
$-112-$

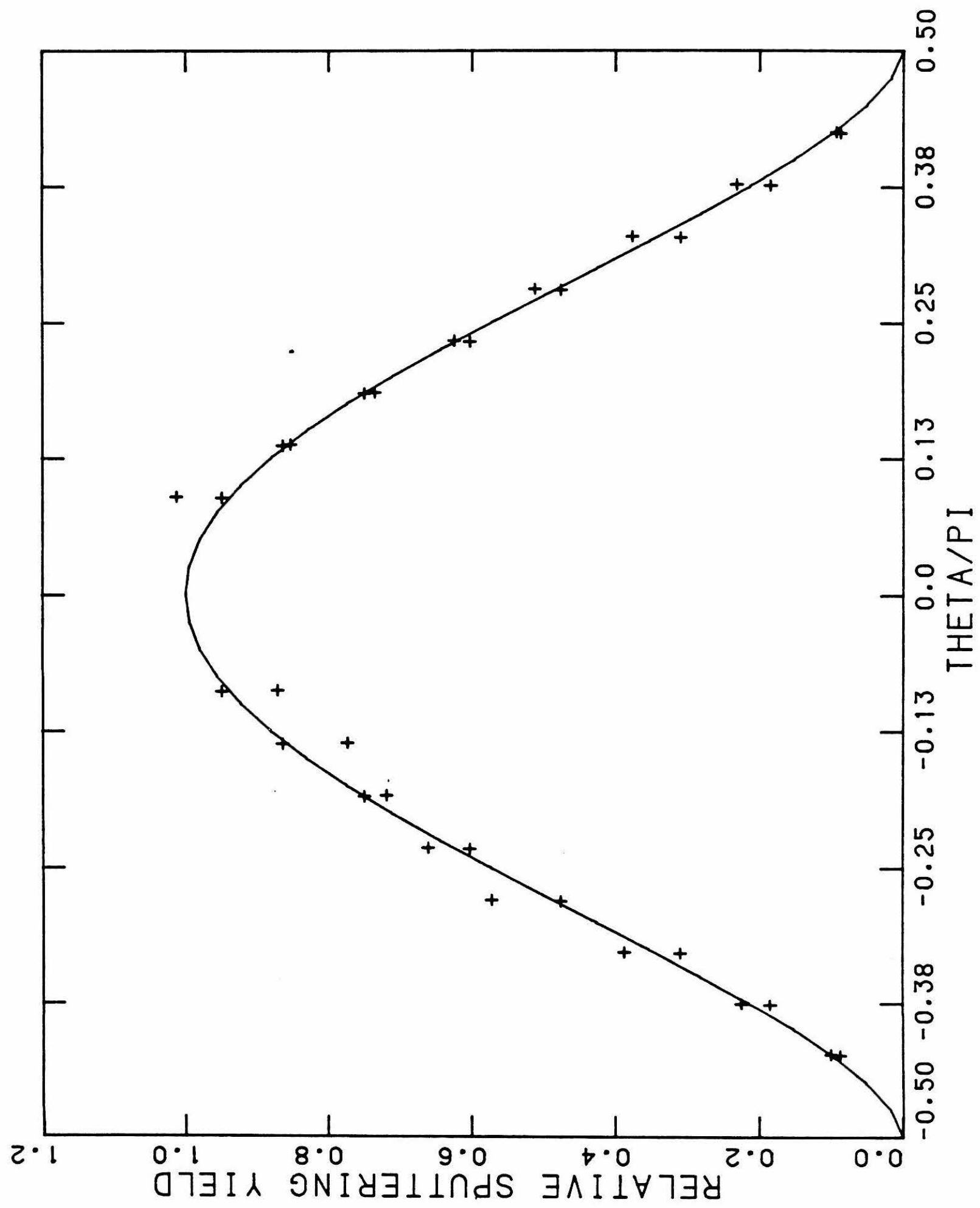

Figure 24 
Figure 25

The normalized differential sputtering yield versus $\theta$ for indium sputtered during the second run of the quickly frozen eutectic target. The total accumulated $\mathrm{Ar}^{+}$at the end of the run was 8.33 mCoulombs. The continuous line is $\cos ^{1 \cdot 3} \theta$. 


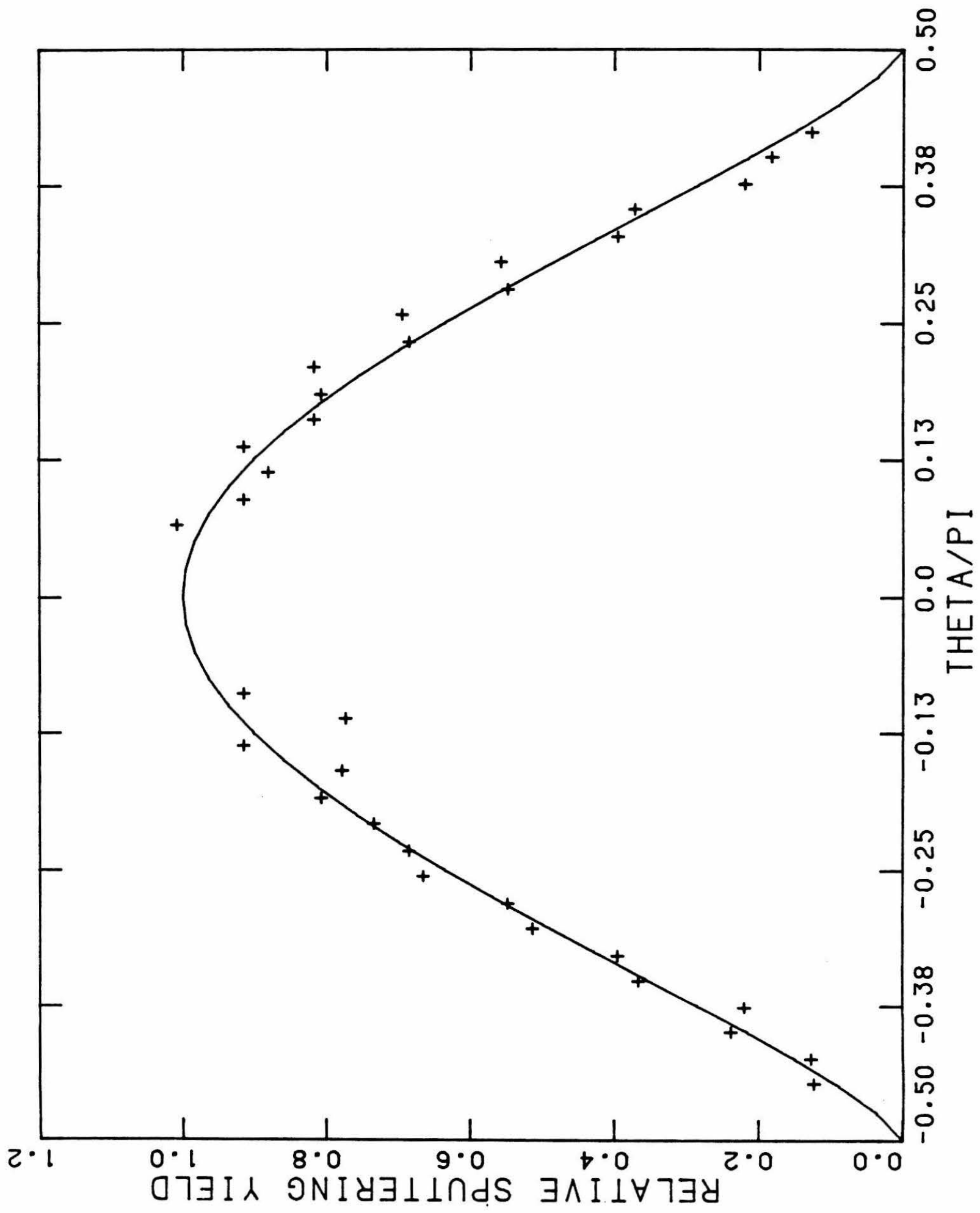

Figure 25 
Figure 26

The normalized differential sputtering yield versus $\theta$ for indium sputtered during the third run of the quickly frozen eutectic target. The total accumulated $\mathrm{Ar}^{+}$at the end of the run was 13.3 mCoulombs. The continuous line is $\cos ^{1 \cdot 2} \theta$. 


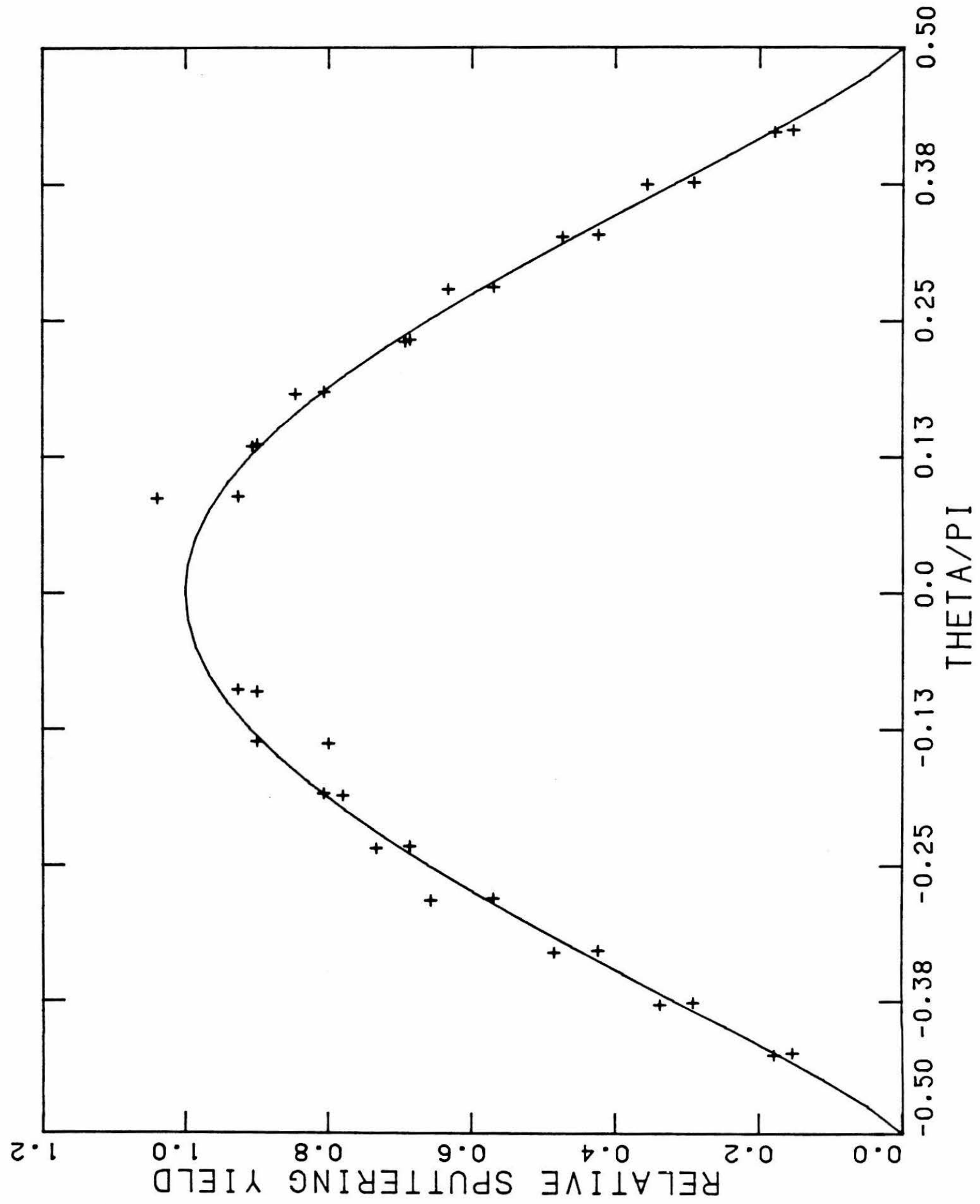

Figure 26 
Figure 27

Photograph of the ion scattering spectroscopy apparatus which was inserted between the analyzing magnet and the target chamber. 


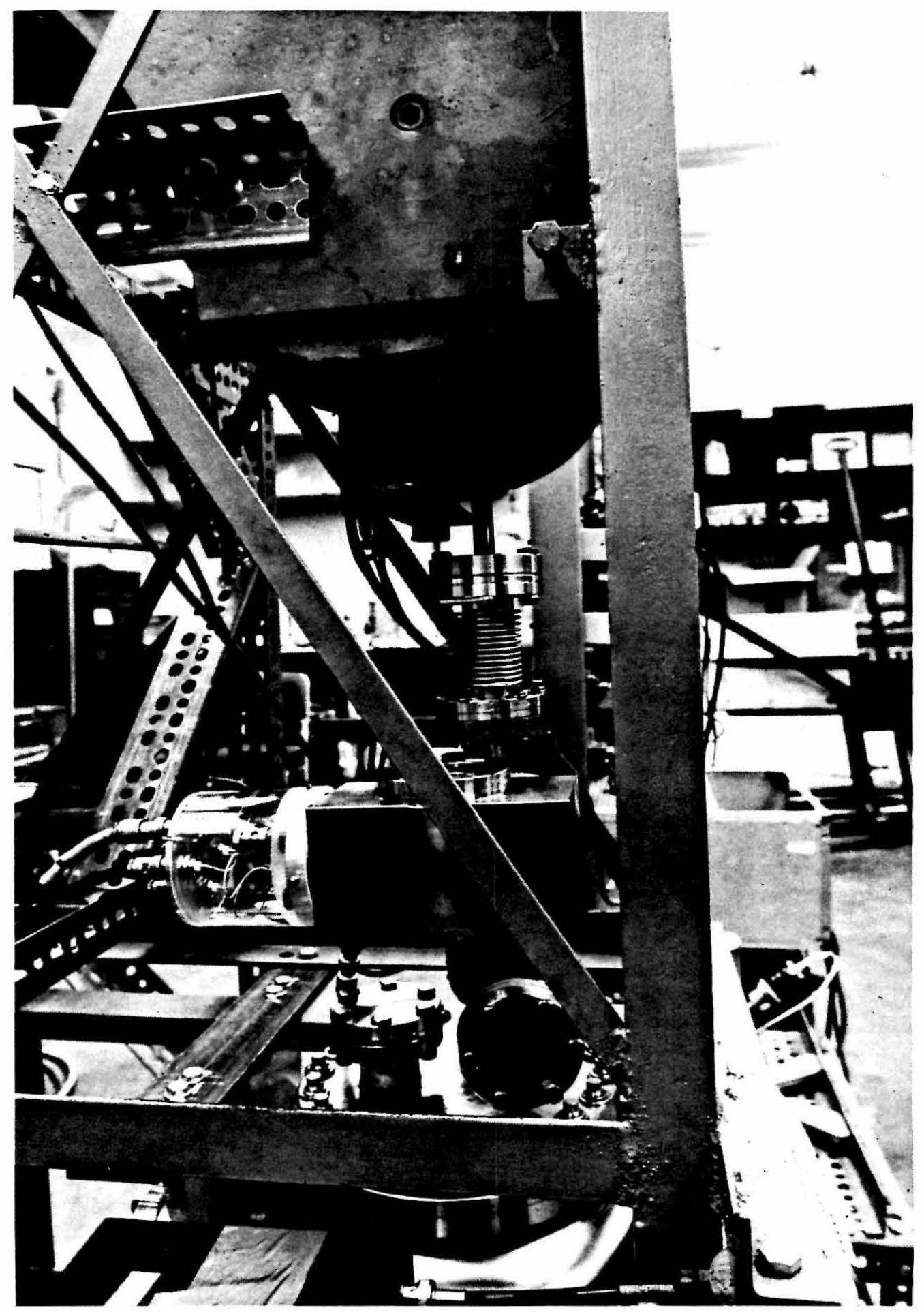


Figure 28

ISS spectrum of a pure solid gallium surface. Each point represents the number of particles detected per $10^{-7}$ Coulomb of incident $2 \mathrm{keV} \mathrm{Ar}^{+}$on target. The detected particles can be sputtered ions as well as scattered $\mathrm{Ar}^{+}$. The peak labeled gallium is comprised of $\mathrm{Ar}^{+}$ions which were scattered from gallium atoms on the surface. 


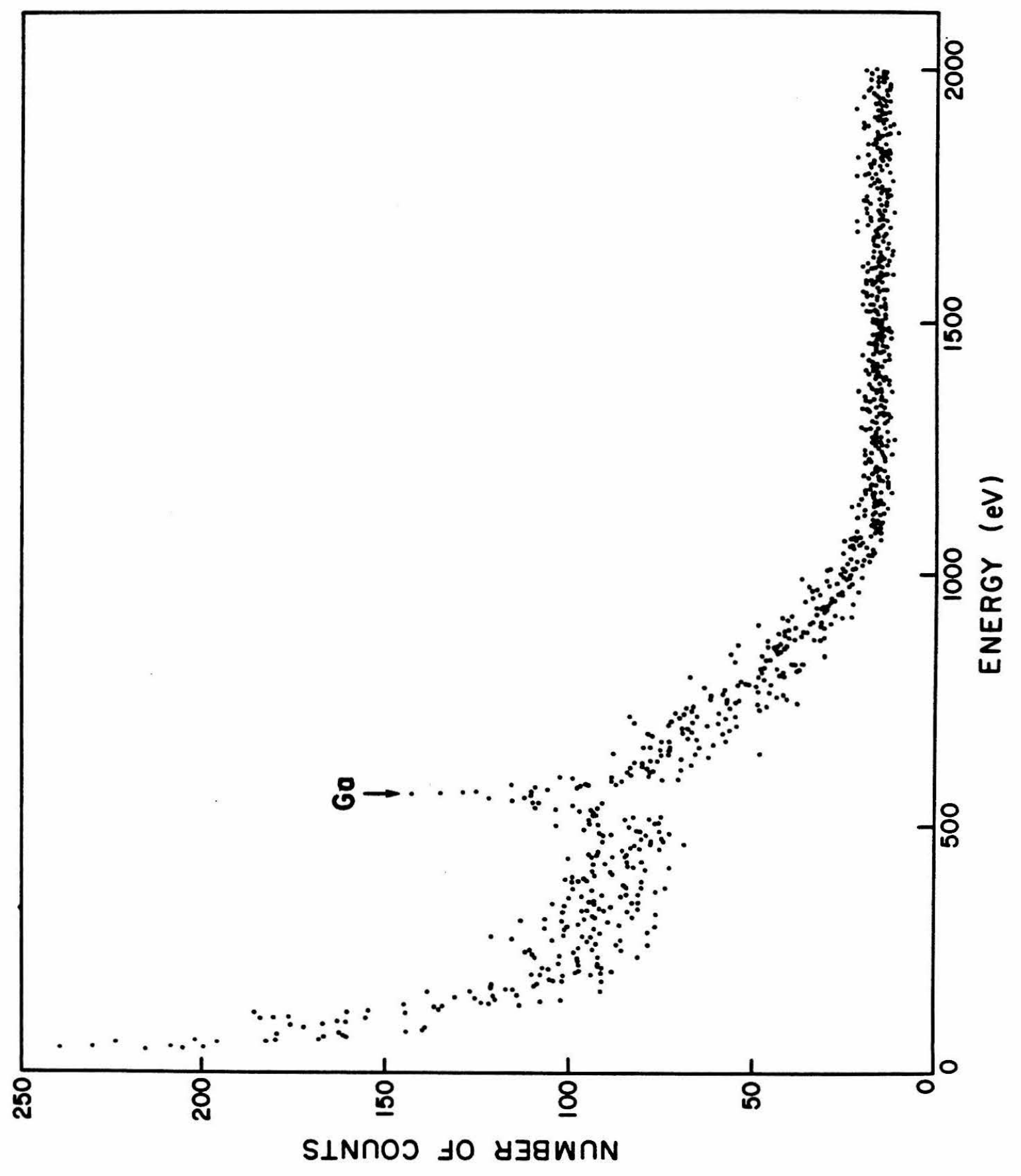

Figure 28 


\section{Figure 29}

ISS spectrum of a pure solid indium surface. Once again, the counts can represent sputtered ions as well as scattered $\mathrm{Ar}^{+}$. The peak labeled indium is comprised of $\mathrm{Ar}^{+}$ions which were scattered from indium atoms on the surface. 


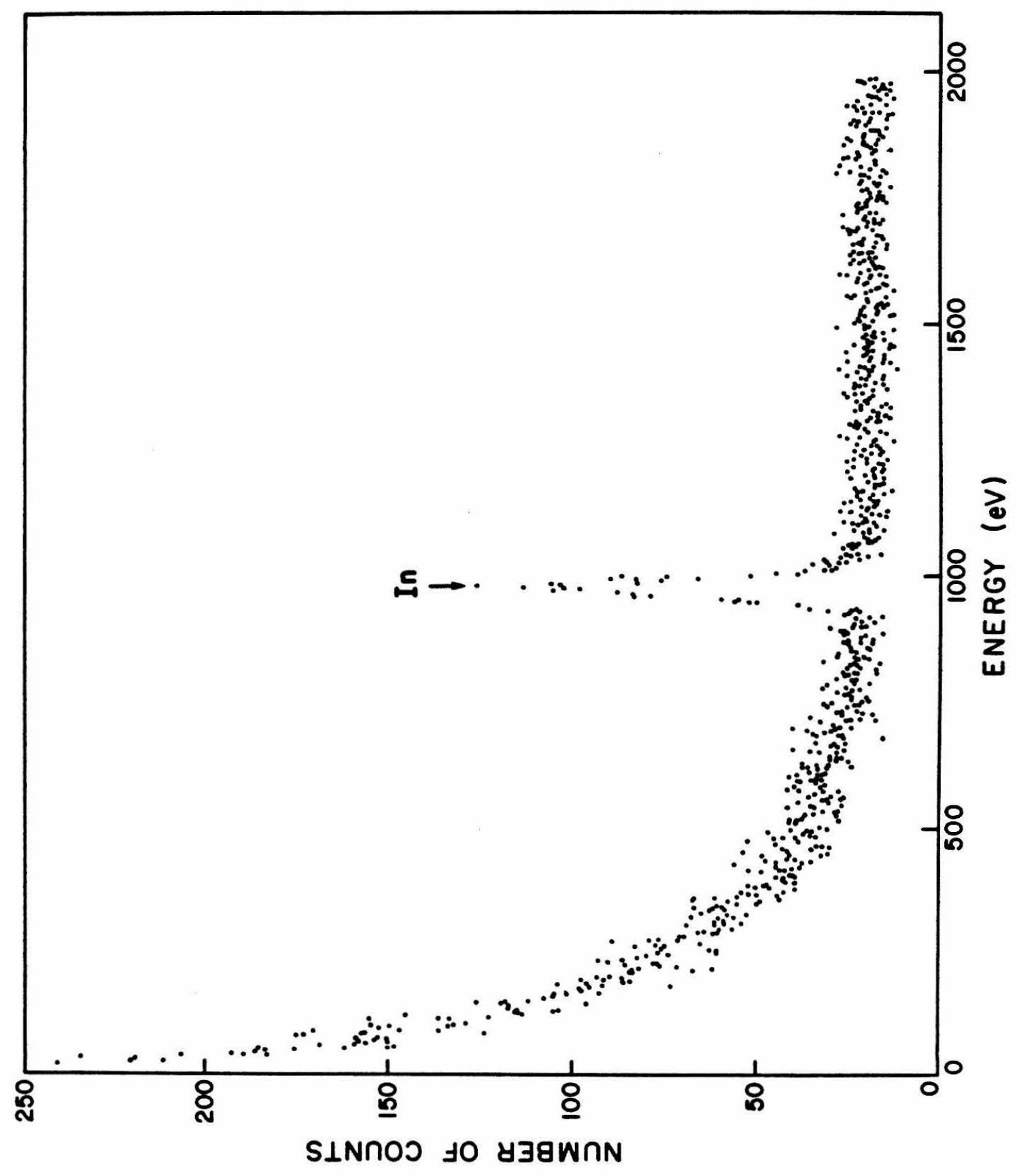

Figure 29 
Figure 30

ISS spectrum of the liquid gallium-indium eutectic alloy. A previous sputter-cleaning exposed some of the tantalum on which the liquid alloy was supported. 


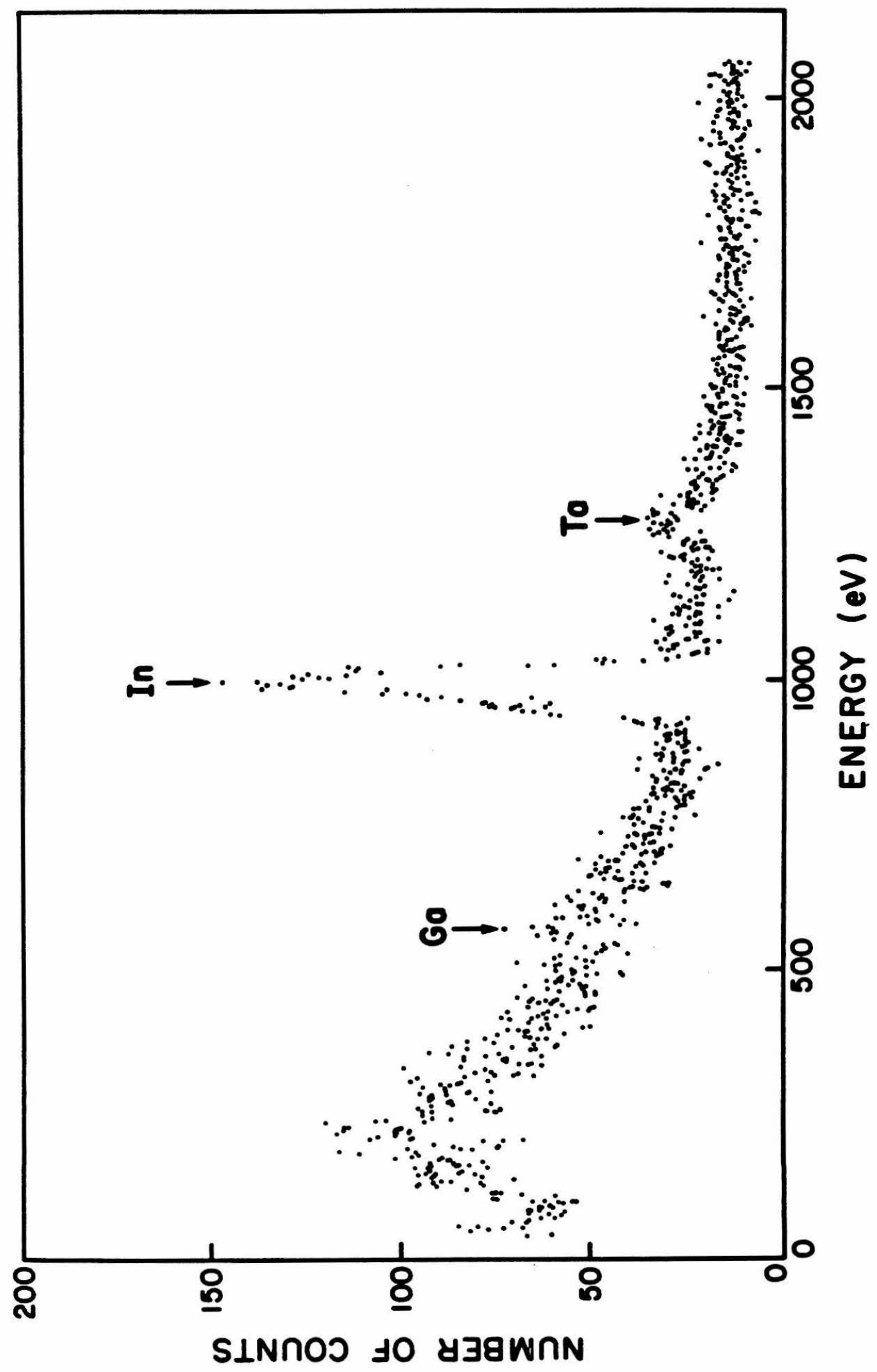


Figure 31

Schematic diagram of the ISS apparatus. The incident beam trajectory is perpendicular to the plane of the diagram. 


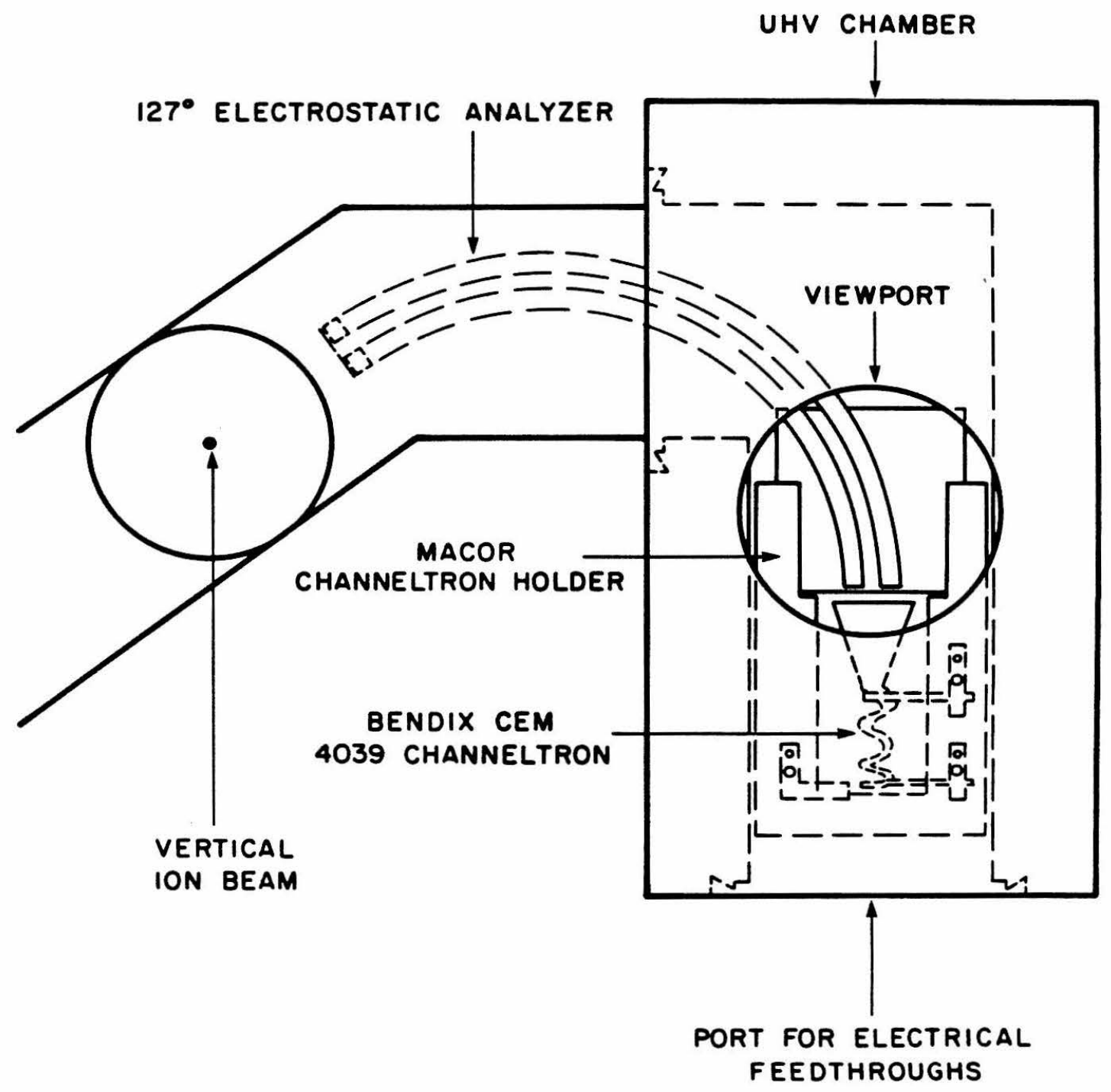

Figure 31 
Figure 32

Photograph corresponding to the point of view taken in the schematic in Figure 31. Part of the electrostatic analyzer can be seen through the viewport. 


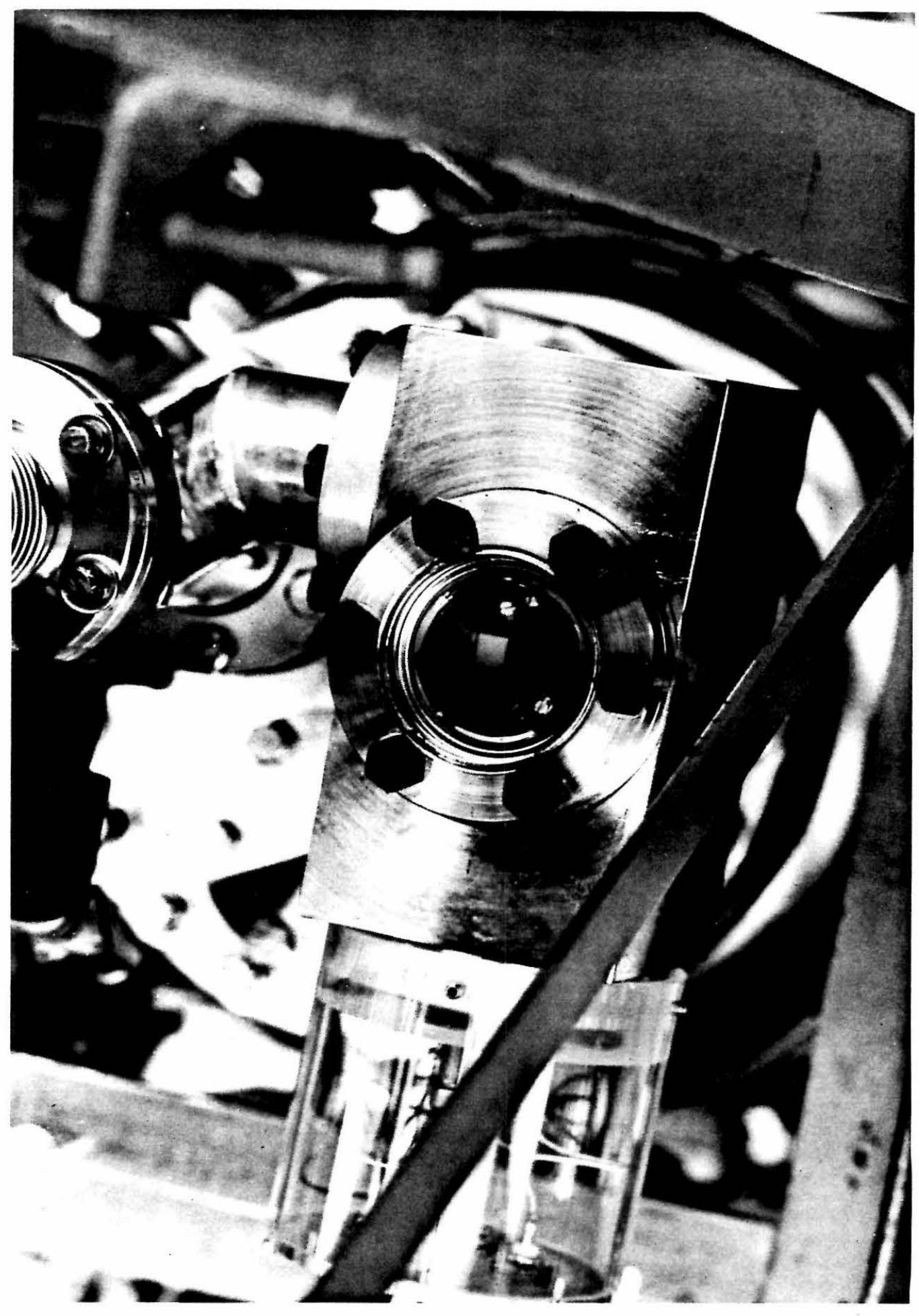


Figure 33

Schematic diagram of the multichannel scaling arrangement used to collect IsS energy spectra. 


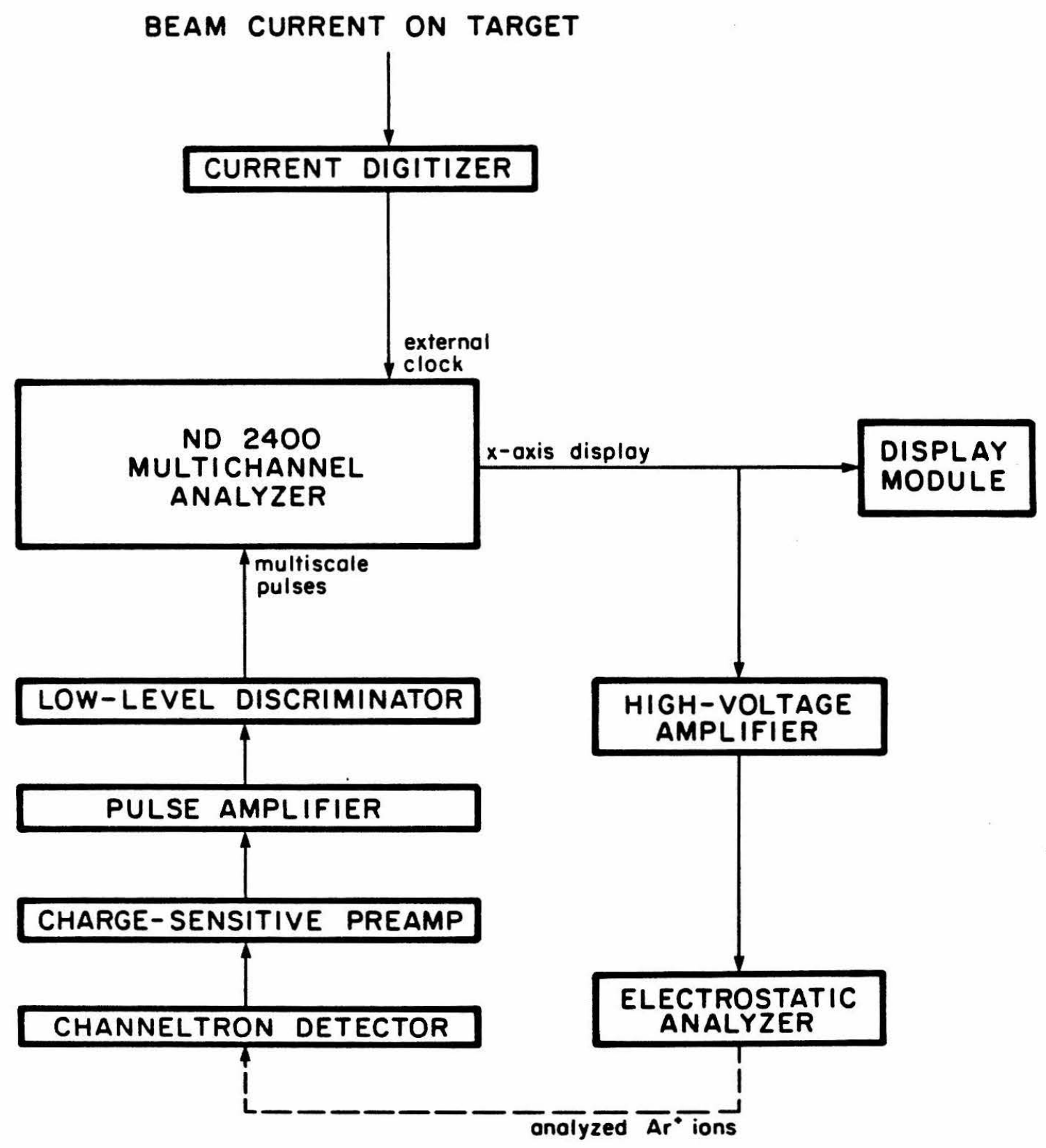

Figure 33 
Figure 34

Circuit diagram of the amplifier used to provide the voltages for the electrostatic analyzer. This amplifier is referred to as "high-voltage" in the schematic in Figure 33 to distinguish it from the amplifier used to amplify and shape the pulses from the channeltron detector. 


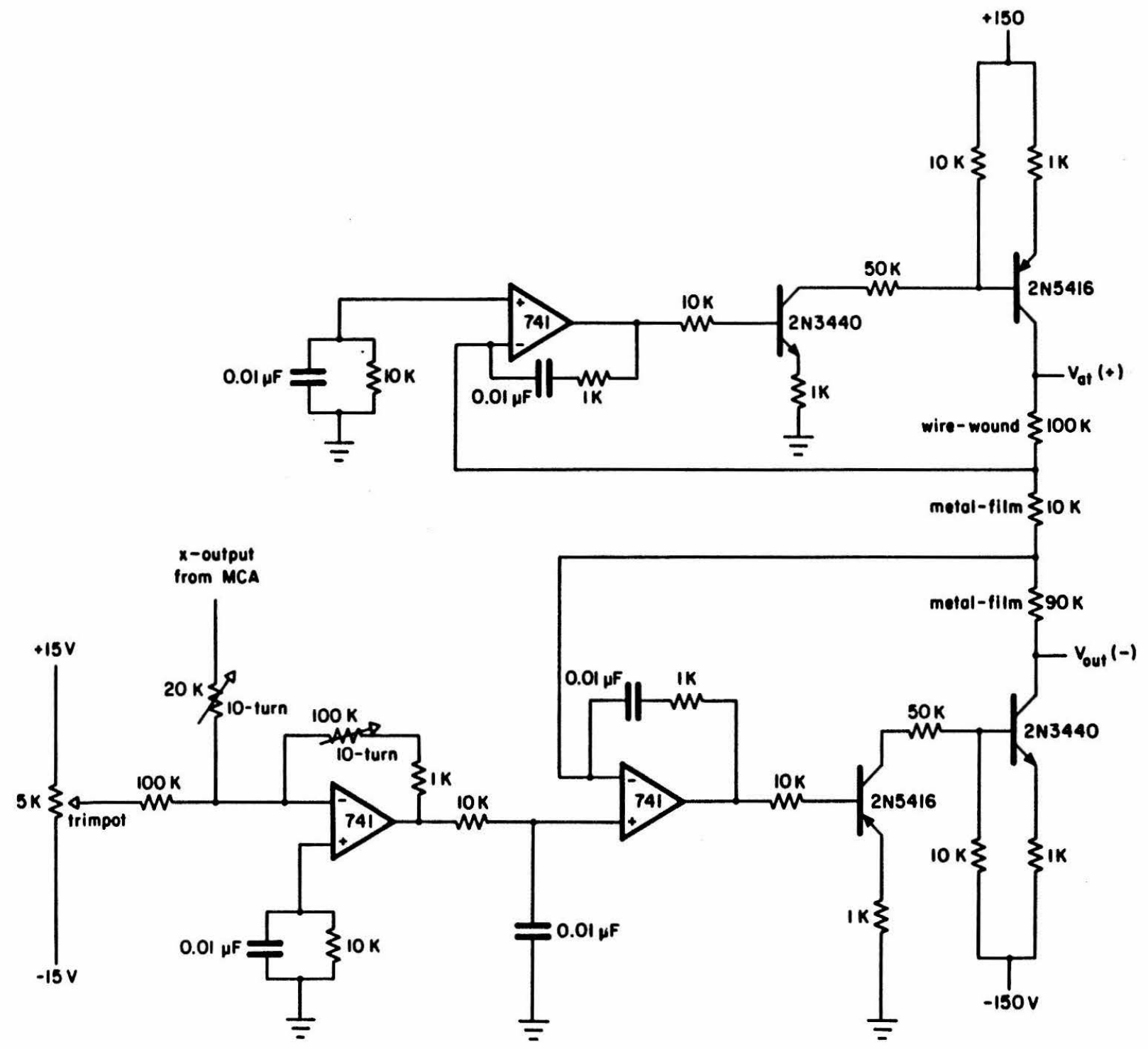

